\title{
MULTIDIMENSIONAL VERSION \\ OF THE RESULTS OF KOMLÓS, MAJOR AND TUSNÁDY FOR VECTORS WITH FINITE EXPONENTIAL MOMENTS
}

\author{
A.YU. ZAITSEV
}

\begin{abstract}
A multidimensional version of the results of Komlós, Major and Tusnády for the Gaussian approximation of the sequence of successive sums of independent random vectors with finite exponential moments is obtained.
\end{abstract}

\section{INTRODUCTION}

The statement of the problem is well known. It is required to construct on a probability space a sequence of independent random vectors $X_{1}, \ldots, X_{n}$ (with given distributions) and a corresponding sequence of independent Gaussian random vectors $Y_{1}, \ldots, Y_{n}$ (this means that $Y_{i}$ has the same mean and the same covariance operator as $\left.X_{i}, i=1, \ldots, n\right)$ so that the quantity

$$
\Delta(X, Y)=\max _{1 \leq k \leq n}\left|\sum_{i=1}^{k} X_{i}-\sum_{i=1}^{k} Y_{i}\right|
$$

would be so small as possible with large probability. Here $|x|=\max \left|x_{j}\right|$, for $x=\left(x_{1}, \ldots, x_{d}\right) \in \mathbb{R}^{d}$. Sometimes we shall use another notation for summands and, by analogy with notation in Sakhanenko $(1984)$, write $\Delta(\tilde{X}, \tilde{Y})=$ $\max _{1 \leq k \leq n}\left|\sum_{i=1}^{k} \tilde{X}_{i}-\sum_{i=1}^{k} \tilde{Y}_{i}\right|$ and so on. In some sense this problem is one of the most important in probability approximations because many well-known probability theorems can be considered as consequences of results about strong approximation of sequences of sums by corresponding Gaussian sequences. This is related to the law of iterated logarithm, to several theorems about large deviations, to the estimates for the rate of convergence of the Prokhorov distance in the invariance principles (see Prokhorov (1956), Skorokhod (1961), Borovkov (1973)) as well as to the Strassen-type (1964) approximations (see, for example, Csörgö and Hall (1984)).

The rate of approximation in the invariance principle was studied by many authors (see, e.g., Prokhorov (1956), Skorokhod (1961), Borovkov (1973), Csörgő and Révész (1975) and the bibliography in Csörgö and Révész (1981),

URL address of the journal: http://www.emath.fr/ps/

Research supported by the Alexander von Humboldt Foundation, by the Russian Foundation of Basic Research, grants 93-11-01454 and 96-01-00672, by grant RFBR-DFG 9601-00096-ge, and by the International Science Foundation, grant R3600.

Received by the journal April 30, 1996. Revised November 18, 1997. Accepted for publication January 16, 1998.

(c) Société de Mathématiques Appliquées et Industrielles. Typeset by $\mathrm{T}_{\mathrm{E} X}$. 
Csörgő and Hall (1984), Shao (1995)). Skorokhod (1961) worked out a powerful method known now as Skorokhod embedding. For a long time it seemed that it is impossible to propose a method which could be better. However, in 1975, Komlós, Major and Tusnády (KMT) (1975-76) worked out a new method of dyadic approximation. With the help of this method they obtained an exhaustive solution of the problem mentioned above for sequences of independent identically distributed random variables. Sakhanenko (1984) generalized and essentially improved KMT results for the case of non-identically distributed random variables.

The first attempts to extend the KMT approximations to the multidimensional case (see Berkes and Philipp (1979), Philipp (1979), Berger (1982), Einmahl $(1987 \mathrm{a}, \mathrm{b}))$ had a partial success only. Comparatively recently U. Einmahl (1989) obtained multidimensional analogs of KMT results which are close to optimal. However, in his results (in general case of vectors with finite exponential moments) there is an unnecessary logarithmic factor. In this paper we improve the result of Einmahl (1989) and obtain multivariate analogs of KMT results.

Notation 1.1. Writing $z \in \mathbb{R}^{d}$ (resp. $\mathbb{C}^{d}$ ), we always keep in mind that $z=\left(z_{1}, \ldots, z_{d}\right)=z_{1} e_{1}+\cdots+z_{d} e_{d}$, where $z_{j} \in \mathbb{R}^{1}$ (resp. $\mathbb{C}^{1}$ ) and the $e_{j}$ are the basis vectors. The scalar product of vectors $x, y \in \mathbb{R}^{d}$ (resp. $\mathbb{C}^{d}$ ) is denoted by $\langle x, y\rangle=x_{1} \bar{y}_{1}+\cdots+x_{d} \bar{y}_{d}$. For $z \in \mathbb{R}^{d}$ (or $\mathbb{C}^{d}$ ) we shall use the Euclidean norm $\|z\|=\langle z, z\rangle^{1 / 2}$ and the maximum norm $|z|=\max _{1 \leq j \leq k}\left|z_{j}\right|$. For $b>0$ we denote $L(b)=\max \{1, \log b\}$. The distribution and the corresponding covariance operator of a random vector $\xi$ will be denoted by $\mathcal{L}(\xi)$ and $\operatorname{cov} \xi$ (or $\operatorname{cov} F$, if $F=\mathcal{L}(\xi)$ ). The letter $I$ will be used for the identity operator. The symbols $c, c_{1}, c_{2}, \ldots$ (without arguments) will be used for absolute positive constants. The letter $c$ can denote different constants when we do not need to fix their numerical values. The numbering of constants starts in each new section again, except Sections 6-9, where we use the common enumeration.

Definition 1.2. (Zaitsev (1986)) Denote by $\mathcal{A}_{d}(\tau)$ a class of probability distributions depending on a parameter $\tau \geq 0$. The class $\mathcal{A}_{d}(\tau)$ consists of $d$-dimensional distributions $F$ for which the function

$$
\varphi(z)=\varphi(F, z)=\log \int_{\mathbb{R}^{d}} e^{\langle z, x\rangle} F\{d x\} \quad(\varphi(0)=0)
$$

is defined and analytic for $\|z\| \tau<1, z \in \mathbb{C}^{d}$, and

$$
\left|d_{u} d_{v}^{2} \varphi(z)\right| \leq\|u\| \tau\langle D v, v\rangle
$$

for all $u, v \in \mathbb{R}^{d}$ and $\|z\| \tau<1$, where $D=\operatorname{cov} F$, and $d_{u} \varphi$ is the derivative of $\varphi$ in the direction $u$.

In Section 2 we consider some properties of classes $\mathcal{A}_{d}(\tau)$. As examples of distributions from $\mathcal{A}_{d}(c \tau)$ one can consider the distributions concentrated on the ball $\mathbf{B}_{\tau}=\left\{x \in \mathbb{R}^{d}:\|x\| \leq \tau\right\}$, more general distributions satisfying Bernstein-type inequality conditions and infinitely divisible distributions 
with spectral measures concentrated on $\mathbf{B}_{\tau}$ (see Zaitsev (1986), pp. 205207 ).

The following Theorem 1.3 is the main result of the paper. It was announced in Zaitsev (1995a, 1996b, 1997) and, in somewhat weakened form, in Zaitsev (1995b).

THeOREM 1.3. Suppose that $\tau \geq 1, \alpha>0$ and $\xi_{1}, \ldots, \xi_{n}$ are random vectors with distributions $\mathcal{L}\left(\xi_{k}\right) \in \mathcal{A}_{d}(\tau), \mathbb{E} \xi_{k}=0$, cov $\xi_{k}=I, k=1, \ldots, n$. Then one can construct on a probability space a sequence of independent random vectors $X_{1}, \ldots, X_{n}$ and a corresponding sequence of independent Gaussian random vectors $Y_{1}, \ldots, Y_{n}$ so that $\mathcal{L}\left(X_{k}\right)=\mathcal{L}\left(\xi_{k}\right), k=1, \ldots, n$, and

$$
\mathbb{E} \exp \left(\frac{c_{1}(\alpha) \Delta(X, Y)}{\tau d^{3} L(d)}\right) \leq \exp \left(c_{2}(\alpha) d^{9 / 4+\alpha} L\left(n / \tau^{2}\right)\right)
$$

where $c_{1}(\alpha), c_{2}(\alpha)$ are positive quantities depending only on $\alpha$.

In a particular case, when $d=1$ and all summands have a common variance, Theorem 1.3 is equivalent to the main result of Sakhanenko (1984). One should take into account that, using the Hölder inequality, we can transfer the factors under exponential sign in the right-hand side of (1.4) to the denominator of the fraction in the left-hand side of (1.4). Naturally, this makes the result weaker. Einmahl (1989) considered multidimensional vectors with identical covariance operators, satisfying multidimensional analogs of Sakhanenko's conditions.

One can easily verify that if a vector $\xi$ has finite exponential moments $\mathbb{E} e^{\langle h, \xi\rangle}$, for $h \in V$, where $V \subset \mathbb{R}^{d}$ is some neighborhood of zero, then $F=\mathcal{L}(\xi) \in \mathcal{A}_{d}(c(F))$. Therefore, Theorem 1.3 can be considered as a generalization and refinement of the main result of KMT (1975-76). In particular, from Theorem 1.3 one can easily derive the following result, obtained by KMT (1975-76) in the one-dimensional case.

Corollary 1.4. Suppose that a random vector $\xi$ has finite exponential moments $\mathbb{E} e^{\langle h, \xi\rangle}$, for $h \in V$, where $V \subset \mathbb{R}^{d}$ is some neighborhood of zero. Then one can construct on a probability space a sequence of independent random vectors $X_{1}, X_{2}, \ldots$ and a corresponding sequence of independent Gaussian random vectors $Y_{1}, Y_{2}, \ldots$ so that $\mathcal{L}\left(X_{k}\right)=\mathcal{L}(\xi), k=1,2, \ldots$, and

$$
\sum_{j=1}^{n} X_{j}-\sum_{j=1}^{n} Y_{j}=O(\log n) \quad \text { a.s. }
$$

An analog of this result was obtained by Einmahl (1989) under additional smoothness-type restrictions on the distributions $\mathcal{L}(\xi)$. Einmahl (1989, Theorem 10 ), has also proved an analog of Theorem 1.3 but only for sufficiently smooth distributions $\mathcal{L}(\xi)$. As it is noted in KMT (1975-76), from the results of Bártfai (1966) it follows that the accuracy of approximation in (1.5) is the best possible.

The following Theorem 1.5 (by means of its consequence, Theorem 9.1) will be used in the proof of Theorems 1.3 and 1.6. However, it is of independent interest because sometimes it can give a more exact dependence of constants on the characteristics of distributions of summands. 
Theorem 1.5. Suppose that $\tau \geq 1, \lambda \geq 1, \alpha>0$ and $\widetilde{\xi}_{1}, \ldots, \widetilde{\xi}_{n}$ are random vectors with distributions $\mathcal{L}\left(\widetilde{\xi}_{k}\right) \in \mathcal{A}_{d}(\tau), \mathbb{E} \widetilde{\xi}_{k}=0$, cov $\widetilde{\xi}_{k}=I, k=1, \ldots, n$. Assume that there exist random vectors $\widetilde{\eta}_{k}$ which have the same moments of the first three orders as the vectors $\widetilde{\xi}_{k}, \mathcal{L}\left(\widetilde{\eta}_{k}\right) \in \mathcal{A}_{d}(\tau)$ and $\mathbb{P}\left\{\left|\widetilde{\eta}_{k}\right| \leq \lambda\right\}=1$, $k=1, \ldots, n$. Then one can construct on a probability space a sequence of independent random vectors $\widetilde{X}_{1}, \ldots, \widetilde{X}_{n}$ and a corresponding sequence of independent Gaussian random vectors $Y_{1}, \ldots, Y_{n}$ so that $\mathcal{L}\left(\widetilde{\xi}_{k}\right)=\mathcal{L}\left(\widetilde{X}_{k}\right)$, $k=1, \ldots, n$, and

$$
\mathbb{E} \exp \left(\frac{c_{3}(\alpha) \Delta(\tilde{X}, Y)}{\lambda d^{3} \tau^{2}}\right) \leq \exp \left(c_{4}(\alpha)\left(\lambda d^{3 / 2} \tau\right)^{3 / 2+\alpha} L(n)\right),
$$

where $c_{3}(\alpha), c_{4}(\alpha)$ are positive quantities depending only on $\alpha$.

The following Theorem 1.6 shows that if all moments of the third order of the vectors $\xi_{1}, \ldots, \xi_{n}$ in Theorem 1.3 are equal to zero, then the dependence of constants on the dimension can be slightly sharpened.

Theorem 1.6. Suppose that $\tau \geq 1, \alpha>0$ and $\xi_{1}, \ldots, \xi_{n}$ are random vectors with distributions $\mathcal{L}\left(\xi_{k}\right) \in \mathcal{A}_{d}(\tau), \mathbb{E} \xi_{k}=0$, cov $\xi_{k}=I, k=1, \ldots, n$. Assume that for all $u, v, w \in \mathbb{R}^{d}$ we have

$$
\mathbb{E}\left\langle\xi_{k}, u\right\rangle\left\langle\xi_{k}, v\right\rangle\left\langle\xi_{k}, w\right\rangle=0, \quad k=1, \ldots, n .
$$

Then one can construct on a probability space a sequence of independent random vectors $X_{1}, \ldots, X_{n}$ and a corresponding sequence of independent Gaussian random vectors $Y_{1}, \ldots, Y_{n}$ so that $\mathcal{L}\left(X_{k}\right)=\mathcal{L}\left(\xi_{k}\right), k=1, \ldots, n$, and

$$
\mathbb{E} \exp \left(\frac{c_{5}(\alpha) \Delta(X, Y)}{d^{3} \tau}\right) \leq \exp \left(c_{6}(\alpha) d^{9 / 4+\alpha} L\left(n / \tau^{2}\right)\right)
$$

where $c_{5}(\alpha), c_{6}(\alpha)$ are positive quantities depending only on $\alpha$.

REMARK 1.7. It is evident that the condition (1.6) is automatically fulfilled if the vectors $\xi_{1}, \ldots, \xi_{n}$ have symmetric distributions.

In Theorems $1.3,1.5$ and 1.6 the random vectors $\xi_{1}, \ldots, \xi_{n}$ and $\widetilde{\xi}_{1}, \ldots, \widetilde{\xi}_{n}$ are, generally speaking, non-identically distributed. However, they have the same covariance operator $I$. Thus, the problem of obtaining an adequate multidimensional generalization of the main result of Sakhanenko (1984) remains open. By the author's opinion, the proof of Theorem 1.3 can be transformed in the proof of an analogous result for vectors with non-identical covariance operators. The conditions $\operatorname{cov} \xi_{j}=I, \tau \geq 1$ can be apparently changed by $B_{j}^{2} \leq \tau^{2}, B_{j}^{2} \leq \lambda \sigma_{j}^{2}$, where $B_{j}^{2}, \sigma_{j}^{2}$ are respectively the maximal and the minimal eigenvalues of $\operatorname{cov} \xi_{j}, j=1, \ldots, n$. The constants will depend, naturally, on $\lambda$, and $n$ should be changed in the right-hand side of (1.4) by the maximal eigenvalue of the covariance operator of the last sum $\xi_{1}+\cdots+\xi_{n}$. The author hopes to consider this situation in a separate paper. Moreover, it is well known that the results similar to Theorems $1.3,1.5$ and 1.6 imply many useful consequences. For example, one can derive estimates in the invariance principle in the case when the summands $X_{j}$ satisfy less restrictive moment conditions (see, e.g., Shao (1995) and the bibliography in Shao (1995)). The author is going to devote to the consequences of Theorems 1.3, 1.5 and 1.6 also a separate publication. 
In Theorems 1.3, 1.5 and 1.6 we consider the case $\tau \geq 1$. Obviously, the assertions of these theorems become stronger for small $\tau$. An analog of Theorem 1.3 in a particular case when the summands have smooth distributions and $\tau$ can be arbitrarily small is obtained in Götze and Zaitsev (1997). Note that for small $\tau$ the distributions of summands are close to Gaussian ones (see Zaitsev (1986)).

The proof of Theorems $1.3,1.5$ and 1.6 consists of several steps, many of which are close to corresponding steps from the proofs of the main results of KMT (1975-76), Sakhanenko (1984) and Einmahl (1989). In Section 2 we introduce the necessary notation and formulate auxiliary results. In particular (see Definition 2.2), we define the classes $\mathcal{R}_{d}(\tau, \varepsilon) \subset \mathcal{A}_{d}(\tau) \times \mathcal{A}_{d}(\tau)$ of pairs of distributions with close cumulants of the second and third orders and discuss the properties of classes $\mathcal{A}_{d}(\tau)$ and $\mathcal{R}_{d}(\tau, 0)$. We define as well the classes $\mathcal{A}_{d}^{*}(\tau, \delta, \rho) \subset \mathcal{A}_{d}(\tau)$ of distributions satisfying some smoothness conditions which guarantee the existence of smooth densities (see Definition 2.12).

The main tool for the proof of Theorems 1.3, 1.5 and 1.6 is the estimates for the closeness of quantiles of one-dimensional conditional distributions contained in Lemmas 3.1 and 4.1. For the proof of Lemmas 3.1 and 4.1 we use Lemmas 2.14 and 2.15 which are completely proved in Zaitsev (1996b), Lemmas 6.1 and 7.1. In Zaitsev (1996b) one can also find a sketch of the proof of Lemmas 3.1 and 4.1. We give the complete proof of these lemmas in Sections 3 and 4. In Lemma 2.14 we give a non-uniform estimate for large deviations in the local CLT for conditional distributions of the last coordinates of vectors with smooth distributions from $\mathcal{A}_{d}(\tau)$ under condition that the first $d-1$ coordinates are fixed (see (2.17)). Analogously, Lemma 2.15 contains a non-uniform estimate for the closeness of conditional densities of smooth distributions from $\mathcal{R}_{d}(\tau, 0)$ (see $(2.23)$ ). Moreover, Lemma 2.15 states that if $w>0$ is separated from zero and from infinity, then the derivative of the logarithm of conditional density at point $w$ is negative. An estimate of this derivative is also presented (see (2.24)). This estimate is essentially used in the proof of Lemma 4.1.

In Lemma 3.1 we give the estimates for the closeness of quantiles in the CLT for conditional distributions of the last coordinates of vectors with smooth distributions from $\mathcal{A}_{d}(\tau)$ under condition that the first $d-1$ co- $^{-}$ ordinates are fixed (see (3.1)). Analogously, in Lemma 4.1 we provide an estimate for the closeness of quantiles of conditional distribution functions of smooth distributions from $\mathcal{R}_{d}(\tau, 0)$ (see (4.1)). The additional information about the closeness of cumulants leads to the better orders of estimates in Lemma 4.1 in comparison with Lemma 3.1.

In Section 5 we describe the KMT (1975-76) scheme of dyadic approximation. This scheme was essentially used by Sakhanenko (1984) and Einmahl (1989). Note, however, that the formulations and the proofs of KMT (1975-76, inequality (2.5)), Sakhanenko (1984, Lemma 1, p. 34) and Einmahl (1989, inequality (4.11)) are close but slightly different. Our approach practically coincides with Einmahl's one. As in KMT (1975-76), Sakhanenko (1984) and Einmahl (1989), we suppose that some independent random vectors with smooth distributions are already constructed and define in- 
dependent random vectors with given distributions by successive constructing sums of blocks of summands, containing $2^{N}, 2^{N-1}, 2^{N-2}, \ldots, 4,2,1$ summands. For this we apply the so-called Rosenblatt (1952) quantile transformation which was already used by Einmahl (1989). This transformation is a natural generalization of the usual one-dimensional quantile transformation which have been applied by KMT (1975-76) and Sakhanenko (1984).

The scheme of the proof of Theorem 1.3 is very close to that of the main result of Sakhanenko (1984). At first we suppose that the Gaussian vectors $Y_{1}, \ldots, Y_{n}, n=2^{N}$, are already constructed and construct the independent vectors which are bounded with probability one, have sufficiently smooth distributions and the same moments of the first, second and third orders as the needed independent random vectors $X_{1}, \ldots, X_{n}$. Then we construct the vectors $X_{1}, \ldots, X_{n}$ in several steps. After each step the number of $X_{j}$ which are not constructed becomes smaller in $2^{p}$ times, where $p$ is a suitably chosen positive integer. In each step we begin with already constructed vectors which are bounded with probability one and have sufficiently smooth distributions and the needed moments up to the third order. Then we construct the vectors such that, in each block of $2^{p}$ summands, only the first vector has the initial bounded smooth distribution. The rest $2^{p}-1$ vectors have the needed distributions $\mathcal{L}\left(\xi_{j}\right)$. These $2^{p}-1$ vectors from each block will be chosen as $X_{j}$ and will be not involved in the next steps of the procedure. The coincidence of third moments will allow us to use more precise estimates of the closeness of quantiles of conditional distributions obtained in Section 4.

In Section 6 we realize the first step of the procedure just described. We estimate the rate of approximation in the case when we construct on a probability space the independent Gaussian vectors $Y_{j}$ and the independent vectors $X_{j}$ with smooth distributions and the needed moments up to the third order. The main result of Section 6 is formulated in Theorem 6.4.

Sections 7 and 8 are devoted to the estimation of the rate of approximation in the case when we begin with the vectors $\tilde{Y}_{1}, \ldots, \widetilde{Y}_{n}, n=2^{N}$, having smooth distribution with bounded supports and with the same moments up to the third order as the needed vectors $\widetilde{X}_{1}, \ldots, \widetilde{X}_{n}$ and construct the vectors $\widetilde{X}_{1}, \ldots, \widetilde{X}_{n}$ in several steps, diminishing after each step the number of $\widetilde{X}_{j}$ which are not constructed in $2^{p}$ times. The scheme of the proof is a modified version of that from Sakhanenko (1984). We use the induction with respect to the number of steps of the procedure and prove that this procedure provides the good estimate for

$$
\delta_{j}(N)=\left|\sum_{k=1}^{j} \tilde{Y}_{k}-\sum_{k=1}^{j} \tilde{X}_{k}\right|
$$

(see $(8.28),(8.94),(8.97))$. It is very important that in (1.8) there is no supremum over $j=1, \ldots, 2^{N}$. This supremum is taken in the last moment only (see (8.98)). At the end of Section 8 we give the proof of Theorem 1.5.

Theorems 1.3 and 1.6 are proved in Section 9. At first we prove an auxiliary result (Theorem 9.1) which is in fact a simple consequence of Theorem 1.5. However, its formulation is more convenient than that of Theorem 1.5 when we investigate the character of dependence of constants of 
the parameters. This is taken into account in the proofs of Theorems 1.3 and 1.6. For the proof of Theorem 1.3 we need also Lemma 9.2 which shows that there exist absolute positive constants $c_{7}, c_{8}$ such that for any distribution $F \in \mathcal{A}_{d}(1)$ with mean zero and $\operatorname{cov} F=I$ there exist a distribution $G \in \mathcal{A}_{d}\left(c_{7}\right)$ with the same moments up to the third order and such that $G\left\{\left\{x:|x| \leq c_{8} L(d)\right\}\right\}=1$. It can be verified that this statement is valid without $L(d)$ if all the moments of the third order of the distribution $F$ are equal to zero. Therefore, the factor $L(d)$ from (1.4) is absent in the denominator of the fraction in the left-hand side of the inequality (1.7) of Theorem 1.6.

\section{Notation AND AUXILIARY RESUlts}

Notation 2.1. Let $\mathcal{F}_{d}$ be the set of all $d$-dimensional probability distributions defined on the $\sigma$-field of Borel subsets of the Euclidean space $\mathbb{R}^{d}$. Let $\mathcal{G}_{d}$ be the collection of all Gaussian distributions from $\mathcal{F}_{d}$. Below $\theta$ symbolizes different quantities not exceeding one in absolute value, $E_{a}$ is the distribution concentrated at a point $a$. By $\widehat{F}(t), t \in \mathbb{R}^{d}$, we denote the characteristic function of a distribution $F \in \mathcal{F}_{d}$. The product of measures is understood as their convolution: $F G=F * G$. By $\mathcal{N}(0, I) \in \mathcal{G}_{d}$ we denote the Gaussian distribution with mean zero and covariance operator $I$. The notation $\Phi(F)$ will be used for the Gaussian distribution whose mean and covariance operator are the same as those of a distribution $F \in \mathcal{F}_{d}$. In onedimensional case we denote by $\Phi_{\sigma}(\cdot)$ the distribution function of the normal law with mean zero and variance $\sigma^{2}$ and by $\varphi_{\sigma}(\cdot)$ the corresponding density. We shall identify the covariance operators with corresponding covariance matrices.

Let $\tau \geq 0, F=\mathcal{L}(\xi) \in \mathcal{A}_{d}(\tau),\|h\| \tau<1, h \in \mathbb{R}^{d}$. Then the conjugate distribution $\bar{F}=\bar{F}(h)$ is defined by

$$
\bar{F}\{d x\}=\left(\mathbb{E} e^{\langle h, \xi\rangle}\right)^{-1} e^{\langle h, x\rangle} F\{d x\}
$$

(sometimes it is called the Cramér transform). Denote by $\bar{\xi}(h)$ a random vector with $\mathcal{L}(\bar{\xi}(h))=\bar{F}(h)$. It is clear that $\bar{F}(0)=F$ and

if $U_{1}, \ldots, U_{n} \in \mathcal{A}_{d}(\tau), \quad\|h\| \tau<1, \quad U=\prod_{j=1}^{n} U_{j}, \quad$ then $\bar{U}(h)=\prod_{j=1}^{n} \bar{U}_{j}(h)$.

Definition 2.2. For $\tau, \varepsilon \geq 0$ denote by $\mathcal{R}_{d}(\tau, \varepsilon)$ the collection of pairs of $d$-dimensional probability distributions $\left(F_{1}, F_{2}\right)$ such that $F_{k} \in \mathcal{A}_{d}(\tau)$, $k=1,2$, and

$$
\begin{aligned}
\left|d_{u} d_{v}^{2} g_{1}(0)-d_{u} d_{v}^{2} g_{2}(0)\right| & \leq \varepsilon\|u\| \tau\langle D v, v\rangle, \\
\left|d_{v}^{2} g_{1}(0)-d_{v}^{2} g_{2}(0)\right| & \leq \varepsilon^{2}\langle D v, v\rangle,
\end{aligned}
$$

for all $u, v \in \mathbb{R}^{d}$, where $D=D_{1}+D_{2}, g_{k}(z)=\varphi\left(F_{k}, z\right), D_{k}=\operatorname{cov} F_{k}$.

REMARK 2.3. If $\varepsilon=0$, then the relation $\left(F_{1}, F_{2}\right) \in \mathcal{R}_{d}(\tau, \varepsilon)$ means that the distributions $F_{1}, F_{2} \in \mathcal{A}_{d}(\tau)$ have identical cumulants of second and third orders. 
Classes $\mathcal{A}_{d}(\tau)$ and $\mathcal{R}_{d}(\tau, \varepsilon)$ have properties which are very convenient for applying. Some of them were considered in Zaitsev (1986, 1988, 1996a).

It is evident that if $\tau_{1}<\tau_{2}$, then $\mathcal{A}_{d}\left(\tau_{1}\right) \subset \mathcal{A}_{d}\left(\tau_{2}\right)$. Moreover, it is easy to see that, for fixed $\tau$, the class $\mathcal{A}_{d}(\tau)$ is closed with respect to convolution: if $F_{1}, F_{2}, \ldots, F_{n} \in \mathcal{A}_{d}(\tau)$, then $F_{1} F_{2} \cdots F_{n} \in \mathcal{A}_{d}(\tau)$. The class $\mathcal{A}_{d}(0)$ coincides with the class $\mathcal{G}_{d}$ of all $d$-dimensional Gaussian distributions. The following inequality was proved in Zaitsev (1986) and can be considered as an estimate of stability of this characterization: if $F \in \mathcal{A}_{d}(\tau), \tau>0$, then $\pi(F, \Phi(F)) \leq$ $c d^{2} \tau L\left(\tau^{-1}\right)$, where $\pi(\cdot, \cdot)$ is the Prokhorov distance. Other useful properties of classes $\mathcal{A}_{d}(\tau)$ and $\mathcal{R}_{d}(\tau, 0)$ are contained in the following Lemmas 2.4, $2.5,2.7,2.8$ and 2.11 .

Lemma 2.4. Suppose that $\tau \geq 0, F \in \mathcal{A}_{d}(\tau), h \in \mathbb{R}^{d},\|h\| \tau<1, F=\mathcal{L}(\xi)$, $D=\operatorname{cov} \xi, D(h)=\operatorname{cov} \bar{\xi}(h)$. $\mathbb{E} \xi=0$. Then

a) for all $u \in \mathbb{R}^{d}$ the following relations are valid:

$$
\begin{aligned}
\langle D(h) u, u\rangle & =\langle D u, u\rangle(1+\theta\|h\| \tau), \\
\log \mathbb{E} e^{\langle h, \xi\rangle} & =\frac{1}{2}\langle D h, h\rangle\left(1+\frac{1}{3} \theta\|h\| \tau\right), \\
\log \mathbb{E} e^{i\langle h, \xi\rangle} & =-\frac{1}{2}\langle D h, h\rangle\left(1+\frac{1}{3} \theta\|h\| \tau\right) ;
\end{aligned}
$$

b) if $\|h\| \tau \leq 1 / 2$, then $\bar{F}(h) \in \mathcal{A}_{d}(2 \tau)$.

Lemma 2.4 is contained in Zaitsev (1986, Lemma 2.1).

Lemma 2.5. Suppose that $\tau \geq 0, F=\mathcal{L}(\xi) \in \mathcal{A}_{d}(\tau), y \in \mathbb{R}^{m}, \alpha \in \mathbb{R}^{1}$, and $A: \mathbb{C}^{d} \rightarrow \mathbb{C}^{m}$ is a linear operator such that $A\left(\mathbb{R}^{d}\right) \subset \mathbb{R}^{m}$. Let $\widetilde{\xi} \in \mathbb{R}^{k}$ be the vector consisting of a subset of coordinates of the vector $\xi$. Then

$$
\begin{gathered}
\mathcal{L}(A \xi+y) \in \mathcal{A}_{m}(\|A\| \tau), \quad \text { where } \quad\|A\|=\sup _{x \in \mathbb{R}^{d},\|x\| \leq 1}\|A x\|, \\
\mathcal{L}(\alpha \xi) \in \mathcal{A}_{d}(|\alpha| \tau), \quad \mathcal{L}(\tilde{\xi}) \in \mathcal{A}_{k}(\tau) .
\end{gathered}
$$

Proof. P1t

$$
\rho(z)=\log \mathbb{E} e^{\langle z, A \xi+y\rangle}=\log \mathbb{E} e^{\left\langle A^{*} z, \xi\right\rangle}+\langle z, y\rangle,
$$

where $A^{*}: \mathbb{C}^{m} \rightarrow \mathbb{C}^{d}$ is the adjoint operator for $A, z \in \mathbb{C}^{m}$. Let $D=\operatorname{cov} \xi$, $M=\operatorname{cov} A \xi$. By virtue of $(1.2),(1.3),(2.7)$, for all $u, v \in \mathbb{R}^{m}, z \in \mathbb{C}^{m}$, $\left\|A^{*} z\right\| \tau<1$, the following relations are valid:

$$
\begin{aligned}
\left|d_{u} d_{v}^{2} \rho(z)\right| & =\left|d_{A^{*} u} d_{A^{*} v}^{2} \varphi(F, w)\right|_{w=A^{*} z} \mid \\
& \leq\left\|A^{*} u\right\| \tau\left\langle D A^{*} v, A^{*} v\right\rangle \leq\left\|A^{*}\right\|\|u\| \tau \mathbb{E}\left\langle\xi-\mathbb{E} \xi, A^{*} v\right\rangle^{2} \\
& =\|A\|\|u\| \tau \mathbb{E}\langle A \xi-\mathbb{E} A \xi, v\rangle^{2}=\|u\| \cdot\|A\| \tau \cdot\langle M v, v\rangle .
\end{aligned}
$$

If $\|z\| \cdot\|A\| \tau<1$ then $\left\|A^{*} z\right\| \tau \leq\left\|A^{*}\right\|\|z\| \tau=\|z\| \cdot\|A\| \tau<1$ and the inequality (2.8) is also valid. From (2.7), (2.8) and from definition of classes $\mathcal{A}_{d}(\tau)$ it follows that $\mathcal{L}(A \xi+y) \in \mathcal{A}_{m}(\|A\| \tau)$. The relations 
$\mathcal{L}(\alpha \xi) \in \mathcal{A}_{d}(|\alpha| \tau), \mathcal{L}(\widetilde{\xi}) \in \mathcal{A}_{k}(\tau)$ follow directly from the statement just proved.

Corollary 2.6. Let the conditions of Lemma 2.5 be satisfied, $\alpha \in \mathbb{R}^{1}$, $\left(F_{1}, F_{2}\right) \in \mathcal{R}_{d}(\tau, 0), F_{j}=\mathcal{L}\left(\xi_{j}\right), y_{j} \in \mathbb{R}^{m}, j=1,2$, and let $\widetilde{\xi}_{1}, \widetilde{\xi}_{2} \in \mathbb{R}^{k}$ be the vectors consisting of identical subsets of coordinates of the vectors $\xi_{1}, \xi_{2}$ respectively. Then

$$
\begin{gathered}
\left(\mathcal{L}\left(A \xi_{1}+y_{1}\right), \mathcal{L}\left(A \xi_{2}+y_{2}\right)\right) \in \mathcal{R}_{m}(\|A\| \tau, 0) \\
\left(\mathcal{L}\left(\alpha \xi_{1}\right), \mathcal{L}\left(\alpha \xi_{2}\right)\right) \in \mathcal{R}_{d}(|\alpha| \tau, 0), \quad\left(\mathcal{L}\left(\widetilde{\xi}_{1}\right), \mathcal{L}\left(\widetilde{\xi}_{2}\right)\right) \in \mathcal{R}_{k}(\tau, 0) .
\end{gathered}
$$

Proof. It is sufficient to use Lemma 2.5, the definition of classes $\mathcal{R}_{d}(\tau, \varepsilon)$ and the corresponding analogs of the relations (2.7), (2.8), taking into account Remark 2.3.

Lemma 2.7. Suppose that $\tau \geq 0, F_{k}=\mathcal{L}\left(\xi^{(k)}\right) \in \mathcal{A}_{d_{k}}(\tau)$, and the vectors $\xi^{(k)}, k=1,2$, are independent. Let $\xi \in \mathbb{R}^{d_{1}+d_{2}}$ be the vector with the first $d_{1}$ coordinates coinciding with those of $\xi^{(1)}$ and with the last $d_{2}$ coordinates coinciding with those of $\xi^{(2)}$. Then $F=\mathcal{L}(\xi) \in \mathcal{A}_{d_{1}+d_{2}}(\tau)$.

Proof. Let $D=\operatorname{cov} \xi, D^{(k)}=\operatorname{cov} \xi^{(k)}, k=1,2$. Using the natural analogous notation, we see that for all $u, v \in \mathbb{R}^{d_{1}+d_{2}}$ and $z \in \mathbb{C}^{d_{1}+d_{2}},\|z\| \tau<1$,

$$
\begin{aligned}
\left|d_{u} d_{v}^{2} \varphi(F, z)\right| & =\left|d_{u} d_{v}^{2}\left(\varphi\left(F_{1}, z^{(1)}\right)+\varphi\left(F_{2}, z^{(2)}\right)\right)\right| \\
& =\left|d_{u^{(1)}} d_{v^{(1)}}^{2} \varphi\left(F_{1}, z^{(1)}\right)+d_{u^{(2)}} d_{v^{(2)}}^{2} \varphi\left(F_{2}, z^{(2)}\right)\right| \\
& \leq\left\|u^{(1)}\right\| \tau\left\langle D^{(1)} v^{(1)}, v^{(1)}\right\rangle+\left\|u^{(2)}\right\| \tau\left\langle D^{(2)} v^{(2)}, v^{(2)}\right\rangle \\
& \leq\|u\| \tau\langle D v, v\rangle .
\end{aligned}
$$

It remains to use the definition of classes $\mathcal{A}_{d}(\tau)$.

Lemma 2.8. Suppose that $\tau \geq 0,\left(\mathcal{L}\left(\xi_{1}^{(k)}\right), \mathcal{L}\left(\xi_{2}^{(k)}\right)\right) \in \mathcal{R}_{d_{k}}(\tau, 0), k=1,2$, and the pairs of vectors $\xi_{j}^{(1)}, \xi_{j}^{(2)}, j=1,2$, are the pairs of independent vectors. Let $\xi_{j} \in \mathbb{R}^{d_{1}+d_{2}}, j=1,2$, be the vectors with the first $d_{1}$ coordinates coinciding with those of $\xi_{j}^{(1)}$ and with the last $d_{2}$ coordinates coinciding with those of $\xi_{j}^{(2)}$. Then $\left(\mathcal{L}\left(\xi_{1}\right), \mathcal{L}\left(\xi_{2}\right)\right) \in \mathcal{R}_{d_{1}+d_{2}}(\tau, 0)$.

Proof. It suffices to apply Lemma 2.7, taking into account Remark 2.3.

REMARK 2.9. Naturally, Lemmas 2.7 and 2.8 can be evidently extended on the case of any finite number of independent vectors.

RemaRK 2.10. Lemma 2.7 can be easily derived from Lemma 2.5, using the completeness of classes $\mathcal{A}_{d}(\tau)$ with respect to convolution.

Lemma 2.11. Let $\tau \geq 0$ and $X_{1}, \ldots, X_{n}$ be independent random vectors with $\mathcal{L}\left(X_{i}\right) \in \mathcal{A}_{d}(\tau), \mathbb{E} X_{i}=0, S_{i}=X_{1}+\cdots+X_{i}$, for $i=1, \ldots, n$. Let $h, t \in \mathbb{R}^{1}, 0 \leq h \tau<1,0 \leq t \tau<1 / 2, \xi=\max _{1 \leq i \leq n}\left|S_{i}\right|$. Denote by $B^{2}$ the maximal eigenvalue of $\operatorname{cov} S_{n}$. Then

$$
\mathbb{E} e^{h\left|S_{n}\right|} \leq 2 d e^{h^{2} B^{2}}, \quad \mathbb{E} e^{t \xi} \leq 3 d e^{4 t^{2} B^{2}}
$$

and

$$
\mathbb{P}\{\xi \geq x\} \leq 2 d \max \left\{\exp \left(-x^{2} / 4 B^{2}\right), \exp (-x / 4 \tau)\right\}, \quad x \geq 0 .
$$


Proof. By Lemma 2.5, the distributions of the coordinates of the vectors $X_{i}$ belong to the class $\mathcal{A}_{1}(\tau)$ and it suffices to prove the statement of the lemma for $d=1$ (recall that $|\cdot|$ means the maximum norm). Using the completeness of the classes $\mathcal{A}_{1}(\tau)$ with respect to convolution, we see that by Lemma 2.4, inequality $(2.5)$,

$$
\mathbb{E} e^{h\left|S_{n}\right|} \leq \mathbb{E} e^{h S_{n}}+\mathbb{E} e^{-h S_{n}} \leq 2 \exp \left(\frac{1}{2} h^{2} B^{2}\left(1+\frac{1}{3} h \tau\right)\right) \leq 2 e^{h^{2} B^{2}} .
$$

Note that the random sequence $\exp \left(h\left|S_{i}\right|\right)$ (indexed by $i$ ) is a positive submartingale. Therefore, by Doob's inequality (see Doob (1953), p. 314) and by $(2.10)$,

$$
\mathbb{P}\{\xi \geq x\}=\mathbb{P}\left\{e^{h \xi} \geq e^{h x}\right\} \leq \mathbb{E} \exp \left(h\left|S_{n}\right|-h x\right) \leq 2 \exp \left(h^{2} B^{2}-h x\right) .
$$

We then deduce (2.9) from (2.11) via the classical Bernstein-Cramér-Chernoff calculation. Integrating by parts and applying (2.11) with $h=2 t$, we get

$$
\mathbb{E} e^{t \xi}=1+\int_{0}^{\infty} t e^{t x} \mathbb{P}\{\xi \geq x\} d x \leq 3 e^{4 t^{2} B^{2}} .
$$

Definition 2.12. Let $\tau \geq 0, \delta>0, \rho>0$. Denote by $\mathcal{A}_{d}^{*}(\tau, \delta, \rho)$ the class of distributions $F \in \mathcal{A}_{d}(\bar{\tau})$ such that for $h \in \mathbb{R}^{d},\|h\| \tau<1$, and for all $v \in \mathbb{R}^{d}$ the following inequalities hold:

$$
\begin{array}{r}
(2 \pi)^{-d} \int_{\rho\|t\| \tau d \geq 1}\left|\widehat{F}_{h}(t)\right| d t \leq \frac{\tau^{2} d^{2}}{\sigma^{2}(2 \pi)^{d / 2}(\operatorname{det} D)^{1 / 2}}, \\
(2 \pi)^{-d} \int_{\rho\|t\| \tau d \geq 1}\left|\langle t, v\rangle \widehat{F}_{h}(t)\right| d t \leq \frac{\left\langle D^{-1} v, v\right\rangle^{1 / 2}}{\delta(2 \pi)^{d / 2}(\operatorname{det} D)^{1 / 2}},
\end{array}
$$

where $F_{h}=\bar{F}(h), D=\operatorname{cov} F$ and $\sigma^{2}>0$ is the minimal eigenvalue of $D$.

According to $(2.1)$, we have (if $F=\mathcal{L}(\xi)$ )

$$
\widehat{F}_{h}(t)=\mathbb{E} e^{\langle i t, \bar{\xi}(h)\rangle}=\left(\mathbb{E} e^{\langle h, \xi\rangle}\right)^{-1} \mathbb{E} e^{\langle h+i t, \xi\rangle} .
$$

Therefore, the conditions (2.12), (2.13) play in this paper the role which is similar to that of Sakhanenko (1984, inequality (49), p. 9) or Einmahl (1989, inequality (1.5)). Note, however, that the condition (2.13) is needed for estimating the derivatives of densities (see (2.24)). Such estimates are necessary for obtaining more precise bounds for the closeness of conditional distributions when the compared distribution have the coinciding third moments.

Notation 2.13. Below for $x=\left(x_{1}, \ldots, x_{d}\right) \in \mathbb{R}^{d}, d \geq 2$, we shall denote by $x^{\prime}$ the vector $x^{\prime}=\left(x_{1}, \ldots, x_{d-1}\right)$ consisting of the first $d-1$ coordinates of $x$. Analogously, we shall use a prime to denote the matrix $D^{\prime}$ of size 
$(d-1) \times(d-1)$ consisting of the first $d-1$ rows and first $d-1$ columns of a matrix $D$ of size $d \times d$. If $F=\mathcal{L}(\xi)$ and $\xi \in \mathbb{R}^{d}$ we shall write $F^{\prime}=\mathcal{L}\left(\xi^{\prime}\right)$.

The following Lemmas 2.14 and 2.15 are completely proved in Zaitsev (1996b, Lemmas 6.1 and 7.1).

Lemma 2.14. Suppose that $\tau \geq 0$,

$$
F=\mathcal{L}(\xi) \in \mathcal{A}_{d}^{*}(\tau, 4,4) \text { and, if } d \geq 2, \quad F^{\prime}=\mathcal{L}\left(\xi^{\prime}\right) \in \mathcal{A}_{d-1}^{*}(\tau, 4,4) .
$$

Assume that $\mathbb{E} \xi=0$ and the last coordinate $\xi_{d}$ of the vector $\xi$ is not correlated with the previous ones. Let $\left.\sigma^{2}=\left\langle D e_{d}, e_{d}\right\rangle=\mathbb{E} \xi_{d}^{2}\right\rangle 0$ be the minimal eigenvalue of $D=\operatorname{cov} F$. Denote by $p(x), x \in \mathbb{R}^{d}$, the probability density of distribution $F$. Let $p^{\prime}\left(x^{\prime}\right), x^{\prime} \in \mathbb{R}^{d-1}, d \geq 2$, be the density of the vector $\xi^{\prime}$. Denote by $p^{(d)}\left(x_{d} \mid x^{\prime}\right)=p(x) / p^{\prime}\left(x^{\prime}\right), x_{d} \in \mathbb{R}^{1}$, the conditional density of the $d^{\text {th }}$ coordinate $\xi_{d}$ of the vector $\xi \in \mathbb{R}^{d}$ for a fixed value of $\xi^{\prime}=x^{\prime}$ (for $d=1$ we define $p^{(1)}\left(x_{1} \mid x^{\prime}\right)=p(x)$ ). Denote by $G$ the corresponding (depending on $\left.x^{\prime}\right)$ one-dimensional conditional distribution.

Then there exist absolute positive constants $c_{1}, \ldots, c_{6}$ such that the following statements are true for $\tau d^{3 / 2} / \sigma \leq c_{1},\left|\left(D^{\prime}\right)^{-1 / 2} x^{\prime}\right| \leq c_{2} \sigma / d^{3 / 2} \tau$.

For $d \geq 2$ there exists a parameter $\overline{h^{\prime}}=h^{\prime}\left(x^{\prime}\right) \in \mathbb{R}^{d-1}$ which gives the solution of the equation $\mathbb{E} \bar{\xi}^{\prime}\left(h^{\prime}\right)=x^{\prime}$. Define the parameter $h \in \mathbb{R}^{d}$ which has the first $d-1$ coordinates coinciding with corresponding coordinates of $h^{\prime}$ and the last $d^{\text {th }}$ coordinate $h_{d}=0$. If $d=1$, we take $h=0$. Denote $y_{0}=$ $\mathbb{E}\left\langle\bar{\xi}(h), e_{d}\right\rangle$. Then

$$
\left|y_{0}\right| \leq 2.88 \tau\left\|\left(D^{\prime}\right)^{-1 / 2} x^{\prime}\right\|^{2} \leq 2.88 \tau \frac{\left\|x^{\prime}\right\|^{2}}{\sigma^{2}},
$$

and, if $|w| \leq \frac{c_{3} \sigma^{2}}{d \tau}$, then for the density $v(w)$ of the distribution $G E_{-y_{0}}$ the following representation is valid:

$$
v(w)=\varphi_{\sigma}(w) \exp \left(\theta c_{4} \frac{\tau}{\sigma}\left(d^{3 / 2}+d\left\|\left(D^{\prime}\right)^{-1 / 2} x^{\prime}\right\|\left(1+\frac{w^{2}}{\sigma^{2}}\right)+\frac{|w|^{3}}{\sigma^{3}}\right)\right),
$$

and

$$
\frac{1}{2 \sqrt{2 \pi} \sigma} \exp \left(-\frac{w^{2}}{\sigma^{2}}\right) \leq v(w) \leq \frac{2}{\sqrt{2 \pi} \sigma} \exp \left(-\frac{w^{2}}{4 \sigma^{2}}\right)
$$

Moreover, for all $w \in \mathbb{R}^{1}$,

$$
v(w) \leq \frac{c_{5}}{\sigma} \exp \left(-\min \left\{\frac{w^{2}}{4 \sigma^{2}}, \frac{c_{6}|w|}{d \tau}\right\}\right) .
$$

Note that for $d=1$ we use in the formulations the natural agreement $\left|\left(D^{\prime}\right)^{-1 / 2} x^{\prime}\right|=\left\|\left(D^{\prime}\right)^{-1 / 2} x^{\prime}\right\|=\left|x^{\prime}\right|=\left\|x^{\prime}\right\|=0$.

Lemma 2.15. Suppose that $\tau \geq 0,\left(F_{1}, F_{2}\right) \in \mathcal{R}_{d}(\tau, 0)$. Let, for $k=1,2$,

$$
F_{k}=\mathcal{L}\left(\xi_{k}\right) \in \mathcal{A}_{d}^{*}(\tau, 4,4) \text { and, if } d \geq 2, \quad F_{k}^{\prime}=\mathcal{L}\left(\xi_{k}^{\prime}\right) \in \mathcal{A}_{d-1}^{*}(\tau, 4,4) \text {. }
$$


Assume that $\mathbb{E} \xi_{1}=\mathbb{E} \xi_{2}=0$ and the last coordinates $\xi_{k, d}$ of the vectors $\xi_{k}$ are not correlated with the previous ones. Let $\sigma^{2}=\left\langle D e_{d}, e_{d}\right\rangle=$ $\mathbb{E} \xi_{k, d}^{2}>0$ be the minimal eigenvalue of the common covariance operator $D=D_{k}=\operatorname{cov} F_{k}$. Let $p_{k}(z), z \in \mathbb{R}^{d}$, be the probability densities of distributions $F_{k}$. Denote by $p_{k}^{\prime}\left(z^{\prime}\right), z^{\prime} \in \mathbb{R}^{d-1}$, the densities of the vectors $\xi_{k}^{\prime}$. Let $p_{k}^{(d)}\left(x_{k, d} \mid x_{k}^{\prime}\right)=p_{k}\left(x_{k}\right) / p_{k}^{\prime}\left(x_{k}^{\prime}\right), x_{k}=\left(x_{k, 1}, \ldots, x_{k, d}\right)$, be the corresponding conditional densities of the $d^{\text {th }}$ coordinates $\xi_{k, d}$ of the vectors $\xi_{k} \in \mathbb{R}^{d}$ for fixed values of $\xi_{k}^{\prime}=x_{k}^{\prime}\left(\right.$ for $d=1$ we denote $\left.p_{k}^{(1)}\left(x_{k, 1} \mid x_{k}^{\prime}\right)=p_{k}\left(x_{k, 1}\right)\right)$. Let $G_{k}$ be the corresponding (depending on $x_{k}^{\prime}$ ) one-dimensional conditional distributions.

Then there exist absolute positive constants $c_{7}, \ldots, c_{11}$ such that the following statements are true for $\tau d^{3 / 2} / \sigma \leq c_{7},\left|\left(D^{\prime}\right)^{-1 / 2} x_{k}^{\prime}\right| \leq c_{8} \sigma / d^{3 / 2} \tau$, $k=1,2$.

For $d \geq 2$ there exist parameters $h_{k}^{\prime}=h_{k}^{\prime}\left(x_{k}^{\prime}\right) \in \mathbb{R}^{d-1}$ giving the solutions of the equations $\mathbb{E} \bar{\xi}_{k}^{\prime}\left(h_{k}^{\prime}\right)=x_{k}^{\prime}$. Define the parameters $h_{k} \in \mathbb{R}^{d}$ which have the first $d-1$ coordinates coinciding with corresponding coordinates of $h_{k}^{\prime}$ and the last $d^{\text {th }}$ coordinates $h_{k, d}=0$. If $d=1$, we take $h_{k}=0$. Let

$$
\Lambda=\left\|\left(D^{\prime}\right)^{-1 / 2} x_{1}^{\prime}-\left(D^{\prime}\right)^{-1 / 2} x_{2}^{\prime}\right\|, \quad \Gamma=\max _{k=1,2}\left\|\left(D^{\prime}\right)^{-1 / 2} x_{k}^{\prime}\right\| .
$$

Denote $y_{k}=\mathbb{E}\left\langle\bar{\xi}_{k}\left(h_{k}\right), \epsilon_{d}\right\rangle$. Then

$$
\left|y_{1}-y_{2}\right| \leq c_{9}\left(\tau^{2} \sigma^{-1} \Gamma^{3}+\tau \Lambda \Gamma\right) \text {. }
$$

Denote by $v_{k}(w)$ the densities of the distributions $G_{k} E_{-y_{k}}$. Then the following representation is valid for $|w| \leq \frac{c_{10} \sigma^{2}}{d \tau}$ :

$$
v_{1}(w)=v_{2}(w) \exp \left(\theta c_{11} \frac{\tau^{2}}{\sigma^{2}}\left(d^{2}+\left(\Gamma^{2}+\sigma \tau^{-1} \Lambda\right)\left(d+\frac{w^{2}}{\sigma^{2}}\right)+\frac{w^{4}}{\sigma^{4}}\right)\right) .
$$

Moreover, for $8 \sigma \leq w \leq \frac{c_{10} \sigma^{2}}{d \tau}$,

$$
\left.\frac{d}{d z} \log v_{k}(z)\right|_{z=w} \leq-\frac{w}{8 \sigma^{2}},\left.\quad \frac{d}{d z} \log v_{k}(z)\right|_{z=-w} \geq \frac{w}{8 \sigma^{2}}, \quad k=1,2 .
$$

\section{Estimates FOR THE ClOSEnESS OF QUANTILES OF CONDITIONAL DISTRIBUTIONS UNDER THE CONDITIONS OF LEMMA 2.14}

Lemma 3.1. Let the conditions of Lemma 2.14 be satisfied. Then there exist absolute positive constants $c_{1}, \ldots, c_{4}$ such that

$$
\Phi_{\sigma}(z-\gamma(z))<\int_{-\infty}^{z} v(y) d y<\Phi_{\sigma}(z+\gamma(z))
$$

for $\frac{\tau d^{3 / 2}}{\sigma} \leq c_{1},\left|\left(D^{\prime}\right)^{-1 / 2} x^{\prime}\right| \leq \frac{c_{2} \sigma}{d^{3 / 2} \tau},|z| \leq \frac{c_{3} \sigma^{2}}{d \tau}$, where

$$
\gamma(z)=c_{4} \tau\left(d^{3 / 2}+d \delta\left(1+\frac{|z|}{\sigma}\right)+\frac{z^{2}}{\sigma^{2}}\right), \quad \delta=\left\|\left(D^{\prime}\right)^{-1 / 2} x^{\prime}\right\| .
$$


The proof of Lemma 3.1 consists of several steps. For each of them we formulate the corresponding lemma.

Lemma 3.2. Let the conditions of Lemma 3.1 be satisfied. Then there exist absolute positive constants $c_{5}, \ldots, c_{8}$ such that if

$$
\frac{\tau d^{3 / 2}}{\sigma} \leq c_{5}, \quad\left|\left(D^{\prime}\right)^{-1 / 2} x^{\prime}\right| \leq \frac{c_{6} \sigma}{d^{3 / 2} \tau}, \quad 4 \sigma \leq z \leq b=\frac{c_{7} \sigma^{2}}{d \tau}
$$

then

$$
\begin{gathered}
\int_{z+\lambda(2 z)}^{b} \varphi_{\sigma}(u) d u \leq I=I(z, b) \stackrel{\text { def }}{=} \int_{z}^{b} v(y) d y \leq \int_{z-\lambda(z)}^{\infty} \varphi_{\sigma}(u) d u, \\
\int_{z+\lambda(2 z)}^{b} \varphi_{\sigma}(u) d u \leq I(-b,-z)=\int_{-b}^{-z} v(y) d y \leq \int_{z-\lambda(z)}^{\infty} \varphi_{\sigma}(u) d u,
\end{gathered}
$$

where

$$
\lambda(z)=c_{8} \tau\left(d^{3 / 2}+d \delta+z\left(d \delta / \sigma+z / \sigma^{2}\right)\right) \leq z / 8 .
$$

Proof. At first we note that, by Lemma 2.14, for $2 \sigma \leq|y| \leq c_{9} \sigma^{2} / d \tau$ we have

$$
v(y)=\varphi_{\sigma}(y) \exp \left(\theta c_{10} \frac{\tau}{\sigma}\left(d^{3 / 2}+\frac{d \delta y^{2}}{\sigma^{2}}+\frac{|y|^{3}}{\sigma^{3}}\right)\right),
$$

if $c_{5}, c_{6}$ are small enough. Recall that

$$
\varphi_{\sigma}(u)=\frac{1}{\sqrt{2 \pi} \sigma} \exp \left(-\frac{u^{2}}{2 \sigma^{2}}\right), \quad u \in \mathbb{R}^{1} .
$$

We take $c_{8}=16 c_{10}$. Thus,

$$
\lambda(u)=16 c_{10} \tau\left(d^{3 / 2}+d \delta+u\left(d \delta / \sigma+u / \sigma^{2}\right)\right) .
$$

Consider the following functions:

$$
f_{-}(u)=u-\lambda(u), \quad f_{+}(u)=u+\lambda(u) .
$$

We shall study some properties of the function $f_{-}(u)$ for $4 \sigma \leq u \leq 2 b$ and of the function $f_{+}(u)$ for $2 \sigma \leq u \leq b$. It is clear that choosing $c_{5}, c_{6}, c_{7}$ to be sufficiently small we can ensure the validity of the relations

$$
\begin{gathered}
2 b \leq c_{9} \sigma^{2} / d \tau \\
0 \leq \lambda(u) \leq u / 8 \quad \text { for } \quad 2 \sigma \leq u \leq 2 b,
\end{gathered}
$$

and, consequently,

$$
\begin{array}{cc}
2 \sigma \leq u / 2 \leq f_{-}(u) \leq u \leq 2 b \leq c_{9} \sigma^{2} / d \tau & \text { for } \quad 4 \sigma \leq u \leq 2 b \\
2 \sigma \leq u \leq f_{+}(u) \leq 2 u \leq 2 b \leq c_{9} \sigma^{2} / d \tau & \text { for } \quad 2 \sigma \leq u \leq b
\end{array}
$$


Moreover, taking again $c_{5}, c_{6}, c_{7}$ to be small enough, we can ensure the validity of the relation

$$
\lambda^{\prime}(u)=16 c_{10} \tau\left(d \delta / \sigma+2 u / \sigma^{2}\right) \leq 1 / 2 \quad \text { for } \quad 2 \sigma \leq u \leq 2 b .
$$

Since $e^{-2 a} \leq 1-a$ for $0 \leq a \leq 1 / 2$, we have

$$
\begin{aligned}
f_{-}^{\prime}(u) & =1-16 c_{10} \tau\left(d \delta / \sigma+2 u / \sigma^{2}\right) \geq \exp \left(-32 c_{10} \tau\left(d \delta / \sigma+2 u / \sigma^{2}\right)\right) \\
& \geq \exp \left(-2 c_{10} \tau u^{2} \sigma^{-2}\left(d \delta / \sigma+2 u / \sigma^{2}\right)\right)
\end{aligned}
$$

provided that $4 \sigma \leq u \leq 2 b$. Analogously,

$$
\begin{aligned}
f_{+}^{\prime}(u) & =1+16 c_{10} \tau\left(d \delta / \sigma+2 u / \sigma^{2}\right) \leq \exp \left(16 c_{10} \tau\left(d \delta / \sigma+2 u / \sigma^{2}\right)\right) \\
& \leq \exp \left(4 c_{10} \tau u^{2} \sigma^{-2}\left(d \delta / \sigma+2 u / \sigma^{2}\right)\right)
\end{aligned}
$$

provided that $2 \sigma \leq u \leq b$. The relations (3.9), (3.10), (3.14) imply that, for $2 \sigma \leq u \leq 2 b$,

$$
0 \leq \lambda^{\prime}(u) \leq 1 / 2, \quad 1 / 2 \leq f_{-}^{\prime}(u) \leq 1, \quad 1 \leq f_{+}^{\prime}(u) \leq 3 / 2 .
$$

Hence, there exist the inverse functions $f_{+}^{-1}(y)$ (for $f_{+}(2 \sigma) \leq y \leq f_{+}(b)$ ) and $f_{-}^{-1}(y)$ (for $f_{-}(4 \sigma) \leq y \leq f_{-}(2 b)$ ). From (3.13) it follows that

$$
y / 2 \leq f_{+}^{-1}(y) \leq y, \quad y \leq f_{-}^{-1}(y) \leq 2 y .
$$

Using (3.10), (3.17), (3.18), one can easily obtain that

$$
\begin{aligned}
& f_{-}\left(f_{-}^{-1}(y)\right)=f_{-}^{-1}(y)-\lambda\left(f_{-}^{-1}(y)\right) \\
& f_{+}\left(f_{+}^{-1}(y)\right)=f_{+}^{-1}(y)+\lambda\left(f_{+}^{-1}(y)\right)
\end{aligned}
$$

and, consequently,

$$
\begin{aligned}
& f_{-}^{-1}(y)=y+\lambda\left(f_{-}^{-1}(y)\right) \leq y+\lambda(2 y), \\
& f_{+}^{-1}(y)=y-\lambda\left(f_{+}^{-1}(y)\right) \geq y-\lambda(y) .
\end{aligned}
$$

Consider the integral $I$ for $4 \sigma \leq z \leq b$. Then, by (3.13), (3.18), we have:

$$
z \geq 4 \sigma \geq f_{+}(2 \sigma), \quad f_{+}^{-1}(z) \geq 2 \sigma, \quad f_{+}^{-1}(b) \leq b
$$

and

$$
f_{-}^{-1}(z) \geq z \geq 4 \sigma, \quad b \leq f_{-}^{-1}(b) \leq 2 b .
$$

By (3.7), (3.8), (3.11), the following estimates hold:

$$
I \leq \int_{z}^{b} \frac{1}{\sqrt{2 \pi} \sigma} \exp \left(-\frac{y^{2}}{2 \sigma^{2}}+c_{10} \frac{\tau}{\sigma}\left(d^{3 / 2}+\frac{d \delta y^{2}}{\sigma^{2}}+\frac{y^{3}}{\sigma^{3}}\right)\right) d y
$$

and

$$
I \geq \int_{z}^{b} \frac{1}{\sqrt{2 \pi} \sigma} \exp \left(-\frac{y^{2}}{2 \sigma^{2}}-c_{10} \frac{\tau}{\sigma}\left(d^{3 / 2}+\frac{d \delta y^{2}}{\sigma^{2}}+\frac{y^{3}}{\sigma^{3}}\right)\right) d y .
$$


In the right-hand side of (3.23) we make the change of variable $y=f_{+}(u)$. It is clear that $d y=f_{+}^{\prime}(u) d u$. Moreover, by (3.9), (3.10),

$y^{2}=(u+\lambda(u))^{2} \geq u^{2}+2 u \lambda(u)=u^{2}+32 c_{10} u \tau\left(d^{3 / 2}+d \delta+u\left(d \delta / \sigma+u / \sigma^{2}\right)\right)$.

From (3.13) it follows that

$$
c_{10} \frac{\tau}{\sigma}\left(d^{3 / 2}+\frac{d \delta y^{2}}{\sigma^{2}}+\frac{y^{3}}{\sigma^{3}}\right) \leq 8 c_{10} \frac{\tau}{\sigma}\left(\frac{d^{3 / 2} u}{\sigma}+\frac{d \delta u^{2}}{\sigma^{2}}+\frac{u^{3}}{\sigma^{3}}\right)
$$

Using (3.8), (3.16), (3.20), (3.21), (3.25), (3.26), we derive from (3.23) that

$$
I \leq \int_{f_{+}^{-1}(z)}^{f_{+}^{-1}(b)} \varphi_{\sigma}(u) d u \leq \int_{z-\lambda(z)}^{\infty} \varphi_{\sigma}(u) d u .
$$

The upper estimate of $I(z, b)$ in $(3.4)$ is proved. It is clear that the upper estimate of $I(-z,-b)$ in $(3.5)$ can be obtained analogously.

In the right-hand side of (3.24) we make the change of variable $y=f_{-}(u)$. It is clear that $d y=f_{-}^{\prime}(u) d u$. Moreover, by virtue of (3.9), (3.10), (3.12), we have

$y^{2}=(u-\lambda(u))^{2} \leq u^{2}-u \lambda(u)=u^{2}-16 c_{10} u \tau\left(d^{3 / 2}+d \delta+u\left(d \delta / \sigma+u / \sigma^{2}\right)\right)$.

From (3.13) it follows that

$$
c_{10} \frac{\tau}{\sigma}\left(d^{3 / 2}+\frac{d \delta y^{2}}{\sigma^{2}}+\frac{y^{3}}{\sigma^{3}}\right) \leq c_{10} \frac{\tau}{\sigma}\left(\frac{d^{3 / 2} u}{\sigma}+\frac{d \delta u^{2}}{\sigma^{2}}+\frac{u^{3}}{\sigma^{3}}\right) .
$$

Using (3.8), (3.15), (3.20), (3.22), (3.28), (3.29), we derive from (3.24) that

$$
I \geq \int_{f_{-}^{-1}(z)}^{f_{-}^{-1}(b)} \varphi_{\sigma}(u) d u \geq \int_{z+\lambda(2 z)}^{b} \varphi_{\sigma}(u) d u .
$$

The lower estimate of $I(z, b)$ in $(3.4)$ is proved. It is evident that the bound for $I(-z,-b)$ can be obtained in a similar way. The inequality (3.6) follows from (3.12).

Lemma 3.3. Let the conditions of Lemma 3.1 be satisfied. Then there exist absolute positive constants $c_{11}, \ldots, c_{14}$ such that, if

$$
\frac{\tau d^{3 / 2}}{\sigma} \leq c_{11}, \quad\left|\left(D^{\prime}\right)^{-1 / 2} x^{\prime}\right| \leq \frac{c_{12} \sigma}{d^{3 / 2} \tau}, \quad 4 \sigma \leq z \leq \frac{c_{13} \sigma^{2}}{d \tau}
$$


then

$$
\int_{z+\mu(2 z)}^{\infty} \varphi_{\sigma}(u) d u<I=I(z, \infty)=\int_{z}^{\infty} v(y) d y<\int_{z-\mu(z)}^{\infty} \varphi_{\sigma}(u) d u
$$

and

$$
\int_{z+\mu(2 z)}^{\infty} \varphi_{\sigma}(u) d u<I(-\infty,-z)=\int_{-\infty}^{-z} v(y) d y<\int_{z-\mu(z)}^{\infty} \varphi_{\sigma}(u) d u
$$

where

$$
\mu(z)=c_{14} \tau\left(d^{3 / 2}+d \delta+z\left(d \delta / \sigma+z / \sigma^{2}\right)\right) \leq z / 4
$$

Proof. Choosing $c_{11}, c_{12}$ to be small enough, we ensure the possibility to estimate the integral $I(b, \infty)$ with the help of inequality (2.19) of Lemma 2.14. By this inequality, for $y>b$ we have

$$
\begin{aligned}
v(y) & \leq \frac{c_{15}}{\sigma} \exp \left(-\min \left\{\frac{y^{2}}{4 \sigma^{2}}, \frac{c_{16} y}{d \tau}\right\}\right) \\
& \leq \frac{c_{15}}{\sigma} \exp \left(-\min \left\{\frac{c_{7} y}{4 d \tau}, \frac{c_{16} y}{d \tau}\right\}\right)=\frac{c_{15}}{\sigma} \exp \left(-\frac{c_{17} y}{d \tau}\right)
\end{aligned}
$$

Thus, we can estimate $I(b, \infty)$ as follows:

$$
\begin{aligned}
I(b, \infty)=\int_{b}^{\infty} v(y) d y & \leq \int_{b}^{\infty} \frac{c_{15}}{\sigma} \exp \left(-\frac{c_{17} y}{d \tau}\right) d y \\
& =\frac{c_{15} d \tau}{c_{17} \sigma} \exp \left(-\frac{c_{17} c_{7} \sigma^{2}}{d^{2} \tau^{2}}\right) .
\end{aligned}
$$

We shall choose $c_{11}, c_{12}, c_{13}$ so that $c_{13}=\min \left\{c_{7} / 2, \sqrt{c_{7} c_{17}}\right\}, c_{11} \leq c_{5}$, $c_{12} \leq c_{6}$. Thus, we can use the inequalities (3.4)-(3.6). From (3.4), (3.36) it follows that

$$
\begin{aligned}
I(z, \infty) & =I(z, b)+I(b, \infty) \\
& \leq \frac{c_{15} d \tau}{c_{17} \sigma} \exp \left(-\frac{c_{17} c_{7} \sigma^{2}}{d^{2} \tau^{2}}\right)+\int_{z-\mu(z)}^{\infty} \varphi_{\sigma}(u) d u-\int_{z-\mu(z)}^{z-\lambda(z)} \varphi_{\sigma}(u) d u
\end{aligned}
$$

and

$$
I(z, \infty) \geq I(z, b) \geq \int_{z+\mu(2 z)}^{\infty}+\int_{z+\lambda(2 z)}^{z+\mu(2 z)}-\int_{b}^{\infty} \varphi_{\sigma}(u) d u .
$$

We take $c_{14}=2 c_{8}$. Taking into account $(3.6),(3.31),(3.34)$, we have:

$$
\begin{array}{rlrl}
\mu(z) & =2 \lambda(z) \leq z / 4, & z-\mu(z) & \geq 3 z / 4 \\
\mu(2 z) & =2 \lambda(2 z) \leq 8 \lambda(z) \leq z, & z+\mu(2 z) \leq 2 z .
\end{array}
$$


Therefore, taking into account that $c_{13}=\min \left\{c_{7} / 2, \sqrt{c_{7} c_{17}}\right\}$, choosing $c_{11}$ to be sufficiently small, and using (3.6), (3.8), (3.31), (3.39), we can ensure the validity of the inequalities

$$
\begin{aligned}
\int_{z-\mu(z)}^{z-\lambda(z)} \varphi_{\sigma}(u) d u & \geq \frac{\lambda(z)}{\sqrt{2 \pi} \sigma} \exp \left(-\frac{z^{2}}{2 \sigma^{2}}\right) \geq \frac{c_{8} \tau d^{3 / 2}}{\sqrt{2 \pi} \sigma} \exp \left(-\frac{c_{13}^{2} \sigma^{2}}{2 d^{2} \tau^{2}}\right) \\
& >\frac{c_{15} d \tau}{c_{17} \sigma} \exp \left(-\frac{c_{17} c_{7} \sigma^{2}}{d^{2} \tau^{2}}\right)
\end{aligned}
$$

and

$$
\begin{aligned}
\int_{z+\lambda(2 z)}^{z+\mu(2 z)} \varphi_{\sigma}(u) d u & \geq \frac{\lambda(2 z)}{\sqrt{2 \pi} \sigma} \exp \left(-\frac{2 z^{2}}{\sigma^{2}}\right) \geq \frac{c_{8} \tau d^{3 / 2}}{\sqrt{2 \pi} \sigma} \exp \left(-\frac{2 c_{13}^{2} \sigma^{2}}{d^{2} \tau^{2}}\right) \\
& >\exp \left(-\frac{c_{7}^{2} \sigma^{2}}{d^{2} \tau^{2}}\right)=\exp \left(-\frac{b^{2}}{4 \sigma^{2}}\right) \geq \int_{b}^{\infty} \varphi_{\sigma}(u) d u
\end{aligned}
$$

The upper bound in (3.32) follows now from (3.37), (3.40). The lower one can be easily derived from (3.38), (3.41). The inequalities (3.33) can be obtained analogously. The inequality (3.34) is contained in (3.39).

Proof of Lemma 3.1. We shall choose $c_{1}, c_{2}, c_{3}$ so small that $c_{1} \leq c_{11}$, $c_{2} \leq c_{12}, c_{3} \leq c_{13}$. Thus, we can use the inequalities (3.32)-(3.34).

Let $0 \leq z \leq 4 \sigma$. It is evident that, if $c_{1}, c_{2}$ are sufficiently small, then from (3.34) it follows that

$$
\mu(8 \sigma) \leq 4 \mu(4 \sigma) \leq c_{18} \tau\left(d^{3 / 2}+d \delta\right) \leq \sigma,
$$

and, by virtue of (3.2) and Lemma 2.14,

$$
v(y)=\varphi_{\sigma}(y) \exp \left(\theta c_{19} \frac{\tau}{\sigma}\left(d^{3 / 2}+d \delta\right)\right) \quad \text { for } \quad|y| \leq 4 \sigma,
$$

where

$$
c_{19} \frac{\tau}{\sigma}\left(d^{3 / 2}+d \delta\right) \leq 1 / 2 .
$$

From $(3.8),(3.43),(3.44)$ it follows that we can write

$$
v(y)=\varphi_{\sigma}(y)\left(1+\theta c_{20} \frac{\tau}{\sigma}\left(d^{3 / 2}+d \delta\right)\right) \quad \text { for } \quad|y| \leq 4 \sigma .
$$

Thus, the integral $I(z, 4 \sigma)$ can be estimated as follows:

$$
I(z, 4 \sigma)=\int_{z}^{4 \sigma} v(y) d y \leq \int_{z}^{4 \sigma} \varphi_{\sigma}(u) d u+c_{20} \frac{\tau}{\sigma}\left(d^{3 / 2}+d \delta\right) .
$$

Analogously,

$$
I(z, 4 \sigma) \geq \int_{z}^{4 \sigma} \varphi_{\sigma}(u) d u-c_{20} \frac{\tau}{\sigma}\left(d^{3 / 2}+d \delta\right) .
$$


Using Lemma 3.3, we see that

$$
I(4 \sigma, \infty)=\int_{4 \sigma}^{\infty} v(y) d y \leq \int_{4 \sigma-\mu(4 \sigma)}^{\infty} \varphi_{\sigma}(u) d u
$$

and

$$
I(4 \sigma, \infty) \geq \int_{4 \sigma+\mu(8 \sigma)}^{\infty} \varphi_{\sigma}(u) d u .
$$

Taking into account (3.8), (3.42), we obtain the inequalities

$$
\int_{4 \sigma-\mu(4 \sigma)}^{4 \sigma} \varphi_{\sigma}(u) d u \leq \frac{e^{-7} \mu(4 \sigma)}{\sqrt{2 \pi} \sigma} \leq \frac{c_{18} e^{-7}}{4 \sqrt{2 \pi}} \frac{\tau}{\sigma}\left(d^{3 / 2}+d \delta\right)
$$

and

$$
\int_{4 \sigma}^{4 \sigma+\mu(8 \sigma)} \varphi_{\sigma}(u) d u \leq \frac{e^{-8} \mu(8 \sigma)}{\sqrt{2 \pi} \sigma} \leq \frac{c_{18} e^{-8}}{\sqrt{2 \pi}} \frac{\tau}{\sigma}\left(d^{3 / 2}+d \delta\right) .
$$

Now from $(3.46),(3.48),(3.50)$ it follows that

$$
I(z, \infty)=\int_{z}^{\infty} v(y) d y<\int_{z}^{\infty} \varphi_{\sigma}(u) d u+c_{21} \frac{\tau}{\sigma}\left(d^{3 / 2}+d \delta\right) .
$$

Analogously, from $(3.47),(3.49),(3.51)$ one can easily derive that

$$
I(z, \infty)>\int_{z}^{\infty} \varphi_{\sigma}(u) d u-c_{22} \frac{\tau}{\sigma}\left(d^{3 / 2}+d \delta\right) .
$$

Put $c_{23}=\sqrt{2 \pi} \max \left\{c_{21} e^{8}, c_{22} e^{25 / 2}\right\}$. It is clear that choosing $c_{1}, c_{2}$ to be sufficiently small we can ensure the validity of the inequality

$$
\phi=c_{23} \tau\left(d^{3 / 2}+d \delta\right) \leq \sigma .
$$

Hence,

$$
z-\phi \geq-\sigma \quad \text { for } \quad z \geq 0 .
$$

Taking into account (3.8), (3.54), (3.55), we see that, for $0 \leq z \leq 4 \sigma$,

$$
\int_{z-\phi}^{z} \varphi_{\sigma}(u) d u \geq \frac{e^{-8} \phi}{\sqrt{2 \pi} \sigma} \geq c_{21} \frac{\tau}{\sigma}\left(d^{3 / 2}+d \delta\right)
$$

and, analogously,

$$
\int_{z}^{z+\phi} \varphi_{\sigma}(u) d u \geq \frac{e^{-25 / 2} \phi}{\sqrt{2 \pi} \sigma} \geq c_{22} \frac{\tau}{\sigma}\left(d^{3 / 2}+d \delta\right) .
$$

Now from $(3.52),(3.53),(3.56),(3.57)$ it follows that

$$
\int_{z+\phi}^{\infty} \varphi_{\sigma}(u) d u<\int_{z}^{\infty} v(y) d y<\int_{z-\phi}^{\infty} \varphi_{\sigma}(u) d u \quad \text { for } \quad 0 \leq z \leq 4 \sigma .
$$

The statement of Lemma 3.1 for $z \geq 0$ can be easily derived from Lemma 3.3 and from $(3.54),(3.58)$. The case $z \leq 0$ can be considered in a similar way. 


\section{ESTIMATES FOR THE ClOSENESS OF QUANTILES OF CONDITIONAL DISTRIBUTIONS UNDER THE CONDITIONS OF LEMMA 2.15}

Lemma 4.1. Let the conditions of Lemma 2.15 be satisfied. Then there exist absolute positive constants $c_{1}, \ldots, c_{4}$ such that

$$
\int_{-\infty}^{z-\phi(z)} v_{1}(y) d y<\int_{-\infty}^{z} v_{2}(y) d y<\int_{-\infty}^{z+\phi(z)} v_{1}(y) d y
$$

for $\frac{\tau d^{3 / 2}}{\sigma} \leq c_{1},\left|\left(D^{\prime}\right)^{-1 / 2} x_{k}^{\prime}\right| \leq \frac{c_{2} \sigma}{d^{2 / 2} \tau}, k=1,2,|z| \leq \frac{c_{3} \sigma^{2}}{d \tau}$, where

$$
\phi(z)=c_{4} \frac{\tau^{2}}{\sigma}\left(d^{2}+\delta\left(d+\frac{|z|}{\sigma}\right)+\frac{|z|^{3}}{\sigma^{3}}\right), \quad \delta=\Gamma^{2}+\sigma \tau^{-1} \Lambda
$$

The proof of Lemma 4.1 consists of several steps. For each of them we formulate the corresponding lemma. For $-\infty \leq z_{1} \leq z_{2} \leq \infty$ we shall denote

$$
J_{k}\left(z_{1}, z_{2}\right)=\int_{z_{1}}^{z_{2}} v_{k}(y) d y, \quad k=1,2 .
$$

Lemma 4.2. Let the conditions of Lemma 4.1 be satisfied. Then there exist absolute positive constants $c_{5}, \ldots, c_{8}$ such that, if

$$
\frac{\tau d^{3 / 2}}{\sigma} \leq c_{5}, \quad\left|\left(D^{\prime}\right)^{-1 / 2} x_{k}^{\prime}\right| \leq \frac{c_{6} \sigma}{d^{3 / 2} \tau}, k=1,2, \quad 10 \sigma \leq z \leq b=\frac{c_{7} \sigma^{2}}{d \tau}
$$

then

$$
\int_{z+\beta(5 z / 4)}^{b} v_{1}(u) d u \leq J=J_{2}(z, b)=\int_{z}^{b} v_{2}(y) d y \leq \int_{z-\beta(z)}^{\infty} v_{1}(u) d u
$$

and

$$
\int_{-b}^{-z-\beta(5 z / 4)} v_{1}(u) d u \leq J_{2}(-b,-z)=\int_{-b}^{-z} v_{2}(y) d y \leq \int_{-\infty}^{-z+\beta(z)} v_{1}(u) d u
$$

where

$$
\beta(z)=c_{8} \tau^{2} \sigma^{-1}\left(d^{2}+d \delta+z\left(\delta / \sigma+z^{2} / \sigma^{3}\right)\right) \leq z / 8 .
$$

Proof. At first we note that, if $c_{5}, c_{6}$ are small enough, then, by Lemma 2.15 and according to (4.2), for $|y| \leq c_{9} \sigma^{2} / d \tau$ we have

$$
v_{2}(y)=v_{1}(y) \exp \left(\theta c_{10} \frac{\tau^{2}}{\sigma^{2}}\left(d^{2}+d \delta+\frac{\delta y^{2}}{\sigma^{2}}+\frac{y^{4}}{\sigma^{4}}\right)\right)
$$


and, for $8 \sigma \leq y \leq \frac{c_{g} \sigma^{2}}{d \tau}, k=1,2$,

$$
\left.\frac{d}{d x} \log v_{k}(x)\right|_{x=y} \leq-\frac{y}{8 \sigma^{2}},\left.\quad \frac{d}{d x} \log v_{k}(x)\right|_{x=-y} \geq \frac{y}{8 \sigma^{2}} .
$$

We take $c_{8}=256 c_{10}$. Thus,

$$
\beta(u)=256 c_{10} \tau^{2} \sigma^{-1}\left(d^{2}+d \delta+u\left(\delta / \sigma+u^{2} / \sigma^{3}\right)\right) .
$$

Consider the following functions:

$$
g_{-}(u)=u-\beta(u), \quad g_{+}(u)=u+\beta(u) .
$$

We shall study some properties of the function $g_{-}(u)$ for $10 \sigma \leq u \leq 2 b$ and of the function $g_{+}(u)$ for $8 \sigma \leq u \leq b$. It is clear that, choosing $c_{5}, c_{6}, c_{7}$ to be sufficiently small and taking into account (4.2), (4.9), (4.10), (2.21), we can ensure the validity of the relations

$$
\begin{gathered}
2 b \leq c_{9} \sigma^{2} / d \tau \\
0 \leq \beta(u) \leq u / 8 \quad \text { for } \quad 8 \sigma \leq u \leq 2 b,
\end{gathered}
$$

and, consequently,

$$
\begin{array}{ll}
8 \sigma \leq 4 u / 5 \leq g_{-}(u) \leq u \leq 2 b \leq c_{9} \sigma^{2} / d \tau & \text { for } \quad 10 \sigma \leq u \leq 2 b \\
8 \sigma \leq u \leq g_{+}(u) \leq 5 u / 4 \leq 2 b \leq c_{9} \sigma^{2} / d \tau & \text { for } \quad 8 \sigma \leq u \leq b
\end{array}
$$

Moreover, taking again $c_{5}, c_{6}, c_{7}$ to be small enough, we can ensure the validity of the relation

$$
\beta^{\prime}(u)=256 c_{10} \tau^{2} \sigma^{-1}\left(\delta / \sigma+3 u^{2} / \sigma^{3}\right) \leq 1 / 2 \quad \text { for } \quad 8 \sigma \leq u \leq 2 b
$$

Since $e^{-2 a} \leq 1-a$ for $0 \leq a \leq 1 / 2$, we have

$$
\begin{aligned}
g_{-}^{\prime}(u) & =1-256 c_{10} \tau^{2} \sigma^{-1}\left(\delta / \sigma+3 u^{2} / \sigma^{3}\right) \\
& \geq \exp \left(-512 c_{10} \tau^{2} \sigma^{-1}\left(\delta / \sigma+3 u^{2} / \sigma^{3}\right)\right) \\
& \geq \exp \left(-6 c_{10} \tau^{2} u^{2} \sigma^{-3}\left(\delta / \sigma+3 u^{2} / \sigma^{3}\right)\right)
\end{aligned}
$$

provided that $10 \sigma \leq u \leq 2 b$. Analogously,

$$
\begin{aligned}
g_{+}^{\prime}(u) & =1+256 c_{10} \tau^{2} \sigma^{-1}\left(\delta / \sigma+3 u^{2} / \sigma^{3}\right) \\
& \leq \exp \left(256 c_{10} \tau^{2} \sigma^{-1}\left(\delta / \sigma+3 u^{2} / \sigma^{3}\right)\right) \\
& \leq \exp \left(4 c_{10} \tau^{2} u^{2} \sigma^{-3}\left(\delta / \sigma+3 u^{2} / \sigma^{3}\right)\right),
\end{aligned}
$$

provided that $8 \sigma \leq u \leq b$. The relations (4.9), (4.10), (4.14) imply that, for $8 \sigma \leq u \leq 2 b$

$$
0 \leq \beta^{\prime}(u) \leq 1 / 2, \quad 1 \leq g_{+}^{\prime}(u) \leq 3 / 2, \quad 1 / 2 \leq g_{-}^{\prime}(u) \leq 1 .
$$


Therefore, there exist the inverse functions $g_{+}^{-1}(y)$ (for $g_{+}(8 \sigma) \leq y \leq g_{+}(b)$ ) and $g_{-}^{-1}(y)$ (for $g_{-}(10 \sigma) \leq y \leq g_{-}(2 b)$ ). From (4.13) it follows that

$$
4 y / 5 \leq g_{+}^{-1}(y) \leq y, \quad y \leq g_{-}^{-1}(y) \leq 5 y / 4 .
$$

Using (4.10), (4.17), (4.18), one can easily obtain that

$$
\begin{aligned}
& g_{-}\left(g_{-}^{-1}(y)\right)=g_{-}^{-1}(y)-\beta\left(g_{-}^{-1}(y)\right), \\
& g_{+}\left(g_{+}^{-1}(y)\right)=g_{+}^{-1}(y)+\beta\left(g_{+}^{-1}(y)\right),
\end{aligned}
$$

and, consequently,

$$
\begin{aligned}
& g_{-}^{-1}(y)=y+\beta\left(g_{-}^{-1}(y)\right) \leq y+\beta(5 y / 4), \\
& g_{+}^{-1}(y)=y-\beta\left(g_{+}^{-1}(y)\right) \geq y-\beta(y) .
\end{aligned}
$$

Consider the integral $J$ for $10 \sigma \leq z \leq b$. Then, by (4.13), (4.18), we have:

$$
z \geq 10 \sigma \geq g_{+}(8 \sigma), \quad g_{+}^{-1}(z) \geq 8 \sigma, \quad g_{+}^{-1}(b) \leq b
$$

and

$$
g_{-}^{-1}(z) \geq z \geq 10 \sigma, \quad b \leq g_{-}^{-1}(b) \leq 5 b / 4 .
$$

By (4.7), (4.11), the following estimates hold:

$$
J \leq \int_{z}^{b} v_{1}(y) \exp \left(c_{10} \frac{\tau^{2}}{\sigma^{2}}\left(d^{2}+d \delta+\frac{\delta y^{2}}{\sigma^{2}}+\frac{y^{4}}{\sigma^{4}}\right)\right) d y
$$

and

$$
J \geq \int_{z}^{b} v_{1}(y) \exp \left(-c_{10} \frac{\tau^{2}}{\sigma^{2}}\left(d^{2}+d \delta+\frac{\delta y^{2}}{\sigma^{2}}+\frac{y^{4}}{\sigma^{4}}\right)\right) d y .
$$

In the right-hand side of (4.23) we make the change of variable $y=g_{+}(u)$. It is clear that $d y=g_{+}^{\prime}(u) d u$. Moreover, using (4.8)-(4.10), (4.13), we see that

$$
\begin{aligned}
\log v_{1}(y) & =\log v_{1}(u)+\left.\beta(u) \frac{d}{d x} \log v_{1}(x)\right|_{x=u+|\theta| \beta(u)} \\
& \leq \log v_{1}(u)-\frac{u \beta(u)}{8 \sigma^{2}} \\
& =\log v_{1}(u)-32 c_{10} \frac{\tau^{2} u}{\sigma^{3}}\left(d^{2}+d \delta+\frac{u \delta}{\sigma}+\frac{u^{3}}{\sigma^{3}}\right)
\end{aligned}
$$

From (4.13) it follows that

$$
c_{10} \frac{\tau^{2}}{\sigma^{2}}\left(d^{2}+d \delta+\frac{\delta y^{2}}{\sigma^{2}}+\frac{y^{4}}{\sigma^{4}}\right) \leq 3 c_{10} \frac{\tau^{2}}{\sigma^{2}}\left(\frac{d^{2} u}{\sigma}+\frac{d \delta u}{\sigma}+\frac{\delta u^{2}}{\sigma^{2}}+\frac{u^{4}}{\sigma^{4}}\right) .
$$


Using (4.16), (4.20), (4.21), (4.25), (4.26), we derive from (4.23) that

$$
J \leq \int_{g_{+}^{-1}(z)}^{g_{+}^{-1}(b)} v_{1}(u) d u \leq \int_{z-\beta(z)}^{\infty} v_{1}(u) d u .
$$

The upper estimate of $J_{2}(z, b)$ in $(4.4)$ is proved. It is clear that the upper estimate of $J_{2}(-z,-b)$ in $(4.5)$ can be obtained analogously.

In the right-hand side of (4.24) we make the change of variable $y=g_{-}(u)$. It is clear that $d y=g_{-}^{\prime}(u) d u$. Moreover, using (4.8)-(4.10), (4.13), we see that

$$
\begin{aligned}
\log v_{1}(y) & =\log v_{1}(u)-\left.\beta(u) \frac{d}{d x} \log v_{1}(x)\right|_{x=u-|\theta| \beta(u)} \\
& \geq \log v_{1}(u)+\frac{(u-\beta(u)) \beta(u)}{8 \sigma^{2}} \geq \log v_{1}(u)+\frac{u \beta(u)}{10 \sigma^{2}} \\
& \geq \log v_{1}(u)+25 c_{10} \frac{\tau^{2} u}{\sigma^{3}}\left(d^{2}+d \delta+\frac{u \delta}{\sigma}+\frac{u^{3}}{\sigma^{3}}\right) .
\end{aligned}
$$

From (4.13) it follows that

$$
c_{10} \frac{\tau^{2}}{\sigma^{2}}\left(d^{2}+d \delta+\frac{\delta y^{2}}{\sigma^{2}}+\frac{y^{4}}{\sigma^{4}}\right) \leq c_{10} \frac{\tau^{2}}{\sigma^{2}}\left(\frac{d^{2} u}{\sigma}+\frac{d \delta u}{\sigma}+\frac{\delta u^{2}}{\sigma^{2}}+\frac{u^{4}}{\sigma^{4}}\right) .
$$

Using (4.15), (4.20), (4.22), (4.28), (4.29), we derive from (4.24) that

$$
J \geq \int_{g_{-}^{-1}(z)}^{g_{-}^{-1}(b)} v_{1}(u) d u \geq \int_{z+\beta(5 z / 4)}^{b} v_{1}(u) d u .
$$

The lower bound for $J_{2}(z, b)$ in $(4.4)$ is proved. It is clear that $J_{2}(-z,-b)$ can be estimated in a similar way. The inequality (4.6) follows from (4.12).

Lemma 4.3. Let the conditions of Lemma 4.1 be satisfied. Then there exist absolute positive constants $c_{11}, \ldots, c_{14}$ such that, if

$$
\frac{\tau d^{3 / 2}}{\sigma} \leq c_{11}, \quad\left|\left(D^{\prime}\right)^{-1 / 2} x_{k}^{\prime}\right| \leq \frac{c_{12} \sigma}{d^{3 / 2} \tau}, k=1,2, \quad 10 \sigma \leq z \leq \frac{c_{13} \sigma^{2}}{d \tau}
$$

then

$$
\int_{z+\nu(5 z / 4)}^{\infty} v_{1}(u) d u<J=J_{2}(z, \infty)=\int_{z}^{\infty} v_{2}(y) d y<\int_{z-\nu(z)}^{\infty} v_{1}(u) d u
$$

and

$$
\int_{-\infty}^{-z-\nu(5 z / 4)} v_{1}(u) d u<J_{2}(-\infty,-z)=\int_{-\infty}^{-z} v_{2}(y) d y<\int_{-\infty}^{-z+\nu(z)} v_{1}(u) d u
$$

where

$$
\nu(z)=c_{14} \tau^{2} \sigma^{-1}\left(d^{2}+d \delta+z\left(\delta / \sigma+z^{2} / \sigma^{3}\right)\right) \leq z / 4 .
$$


Proof. Choosing $c_{11}, c_{12}$ to be small enough, we ensure the possibility to estimate the integrals $J_{k}(b, \infty), k=1,2$, with the help of inequality (2.19) of Lemma 2.14. By virtue of (2.19), we have (for $y>b, k=1,2$ )

$$
\begin{aligned}
v_{k}(y) & \leq \frac{c_{15}}{\sigma} \exp \left(-\min \left\{\frac{y^{2}}{4 \sigma^{2}}, \frac{c_{16} y}{d \tau}\right\}\right) \\
& \leq \frac{c_{15}}{\sigma} \exp \left(-\min \left\{\frac{c_{7} y}{4 d \tau}, \frac{c_{16} y}{d \tau}\right\}\right)=\frac{c_{15}}{\sigma} \exp \left(-\frac{c_{17} y}{d \tau}\right) .
\end{aligned}
$$

Thus, we can estimate $J_{k}(b, \infty)$ as follows:

$$
\begin{aligned}
J_{k}(b, \infty)=\int_{b}^{\infty} v_{k}(y) d y & \leq \int_{b}^{\infty} \frac{c_{15}}{\sigma} \exp \left(-\frac{c_{17} y}{d \tau}\right) d y \\
& =\frac{c_{15} d \tau}{c_{17} \sigma} \exp \left(-\frac{c_{17} c_{7} \sigma^{2}}{d^{2} \tau^{2}}\right) .
\end{aligned}
$$

We shall choose $c_{11}, c_{12}, c_{13}$ so that

$c_{11} \leq \min \left\{c_{5}, c_{18}\right\}, c_{12} \leq \min \left\{c_{6}, c_{19}\right\}, c_{13}=\min \left\{c_{7}, \frac{\sqrt{c_{7} c_{17}}}{2}, \frac{2 c_{20}}{3}\right\}$,

where $c_{18}, c_{19}, c_{20}$ are the constants $c_{1}, c_{2}, c_{3}$ from Lemma 2.14. Thus, we can use the inequalities (2.18) for $0 \leq|w| \leq 3 c_{13} \sigma^{2} / 2 d \tau$ (with the change of $v(\cdot)$ by $\left.v_{k}(\cdot), k=1,2\right)$ and the inequalities (4.4)-(4.6). From (4.4) and (4.36) it follows that

$$
\begin{aligned}
J_{2}(z, \infty) & =J_{2}(z, b)+J_{2}(b, \infty) \\
& \leq \frac{c_{15} d \tau}{c_{17} \sigma} \exp \left(-\frac{c_{17} c_{7} \sigma^{2}}{d^{2} \tau^{2}}\right)+\int_{z-\nu(z)}^{\infty} v_{1}(u) d u-\int_{z-\nu(z)}^{z-\beta(z)} v_{1}(u) d u
\end{aligned}
$$

and

$$
J_{2}(z, \infty) \geq J_{2}(z, b) \geq \int_{z+\nu(5 z / 4)}^{\infty}+\int_{z+\beta(5 z / 4)}^{z+\nu(5 z / 4)}-\int_{b}^{\infty} v_{1}(u) d u .
$$

We take $c_{14}=2 c_{8}$. Taking into account the relations (4.3), (4.6), (4.31), (4.34) and (4.37), we have:

$$
\begin{array}{rlrl}
\nu(z) & =2 \beta(z) \leq z / 4, & z-\nu(z) & \geq 3 z / 4, \\
\nu(5 z / 4) & =2 \beta(5 z / 4) \leq 4 \beta(z) \leq z / 2, & z+\nu(5 z / 4) \leq 3 z / 2 .
\end{array}
$$

Therefore, using (4.6), (4.31), (4.36), (4.37), (4.40), (2.18) and choosing $c_{11}$ to be sufficiently small, we can ensure the validity of the inequalities

$$
\begin{aligned}
\int_{z-\nu(z)}^{z-\beta(z)} v_{1}(u) d u & \geq \frac{\beta(z)}{2 \sqrt{2 \pi} \sigma} \exp \left(-\frac{z^{2}}{\sigma^{2}}\right) \geq \frac{c_{8} \tau^{2} d^{2}}{2 \sqrt{2 \pi} \sigma^{2}} \exp \left(-\frac{c_{13}^{2} \sigma^{2}}{d^{2} \tau^{2}}\right) \\
& >\frac{c_{15} d \tau}{c_{17} \sigma} \exp \left(-\frac{c_{17} c_{7} \sigma^{2}}{d^{2} \tau^{2}}\right)
\end{aligned}
$$


and

$$
\begin{aligned}
\int_{z+\beta(5 z / 4)}^{z+\nu(5 z / 4)} v_{1}(u) d u & \geq \frac{\beta(5 z / 4)}{2 \sqrt{2 \pi} \sigma} \exp \left(-\frac{9 z^{2}}{4 \sigma^{2}}\right) \geq \frac{c_{8} \tau^{2} d^{2}}{2 \sqrt{2 \pi} \sigma^{2}} \exp \left(-\frac{9 c_{13}^{2} \sigma^{2}}{4 d^{2} \tau^{2}}\right) \\
& >\frac{c_{15} d \tau}{c_{17} \sigma} \exp \left(-\frac{c_{17} c_{7} \sigma^{2}}{d^{2} \tau^{2}}\right) \geq \int_{b}^{\infty} v_{1}(u) d u
\end{aligned}
$$

The upper bound in (4.32) follows now from (4.38), (4.41). The lower one can be easily derived from (4.39), (4.42). The inequalities (4.33) can be obtained analogously. The inequality (4.34) is contained in (4.40).

Proof of Lemma 4.1. We shall choose $c_{1}, c_{2}, c_{3}$ so small that $c_{1} \leq c_{11}$, $c_{2} \leq c_{12}, c_{3} \leq c_{13}$. Thus, we can use the inequalities (4.32)-(4.34).

Let $0 \leq z \leq 10 \sigma$. It is evident that, if $c_{1}, c_{2}$ are small enough, then from (4.2), (4.34), (2.21) it follows that

$$
\nu(25 \sigma / 2) \leq 2 \nu(10 \sigma) \leq c_{21} \tau^{2} \sigma^{-1}\left(d^{2}+d \delta\right) \leq \sigma,
$$

and, by virtue of (4.2) and Lemma 2.15,

$$
v_{2}(y)=v_{1}(y) \exp \left(\theta c_{22} \frac{\tau^{2}}{\sigma^{2}}\left(d^{2}+d \delta\right)\right) \quad \text { for } \quad|y| \leq 10 \sigma
$$

where

$$
c_{22} \frac{\tau^{2}}{\sigma^{2}}\left(d^{2}+d \delta\right) \leq 1 / 2 .
$$

From (4.44), (4.45) it follows that we can write

$$
v_{2}(y)=v_{1}(y)\left(1+\theta c_{23} \frac{\tau^{2}}{\sigma^{2}}\left(d^{2}+d \delta\right)\right) \quad \text { for } \quad|y| \leq 10 \sigma .
$$

Thus, the integral $J_{2}(z, 10 \sigma)$ can be estimated as follows:

$$
J_{2}(z, 10 \sigma)=\int_{z}^{10 \sigma} v_{2}(y) d y \leq \int_{z}^{10 \sigma} v_{1}(y) d y+c_{23} \frac{\tau^{2}}{\sigma^{2}}\left(d^{2}+d \delta\right) .
$$

Analogously,

$$
J_{2}(z, 10 \sigma) \geq \int_{z}^{10 \sigma} v_{1}(y) d y-c_{23} \frac{\tau^{2}}{\sigma^{2}}\left(d^{2}+d \delta\right) .
$$

Using Lemma 4.3, we see that

$$
J_{2}(10 \sigma, \infty)=\int_{10 \sigma}^{\infty} v_{2}(y) d y \leq \int_{10 \sigma-\nu(10 \sigma)}^{\infty} v_{1}(y) d y
$$

and

$$
J_{2}(10 \sigma, \infty) \geq \int_{10 \sigma+\nu(25 \sigma / 2)}^{\infty} v_{1}(y) d y
$$


Taking into account (4.43), choosing $c_{1}, c_{2}, c_{3}$ to be small enough and applying the inequality (2.18) of Lemma 2.14, we obtain the inequalities

$$
\begin{aligned}
\int_{10 \sigma-\nu(10 \sigma)}^{10 \sigma} v_{1}(y) d y & \leq \int_{10 \sigma-\nu(10 \sigma)}^{10 \sigma} \frac{2}{\sqrt{2 \pi} \sigma} \exp \left(-\frac{y^{2}}{4 \sigma^{2}}\right) d y \\
& \leq \frac{2 e^{-22} \nu(10 \sigma)}{\sqrt{2 \pi} \sigma} \leq \frac{c_{21} e^{-22}}{\sqrt{2 \pi}} \frac{\tau^{2}}{\sigma^{2}}\left(d^{2}+d \delta\right)
\end{aligned}
$$

and

$$
\begin{aligned}
\int_{10 \sigma}^{10 \sigma+\nu(25 \sigma / 2)} v_{1}(y) d y & \leq \int_{10 \sigma}^{10 \sigma+\nu(25 \sigma / 2)} \frac{2}{\sqrt{2 \pi} \sigma} \exp \left(-\frac{y^{2}}{4 \sigma^{2}}\right) d y \\
& \leq \frac{2 e^{-25} \nu(25 \sigma / 2)}{\sqrt{2 \pi} \sigma} \leq \frac{2 c_{21} e^{-25}}{\sqrt{2 \pi}} \frac{\tau^{2}}{\sigma^{2}}\left(d^{2}+d \delta\right) .
\end{aligned}
$$

Now from $(4.47),(4.49),(4.51)$ it follows that

$$
J_{2}(z, \infty)=\int_{z}^{\infty} v_{2}(y) d y<\int_{z}^{\infty} v_{1}(y) d y+c_{24} \frac{\tau^{2}}{\sigma^{2}}\left(d^{2}+d \delta\right) .
$$

Analogously, from $(4.48),(4.50),(4.52)$ one can easily derive that

$$
J_{2}(z, \infty)>\int_{z}^{\infty} v_{1}(y) d y-c_{25} \frac{\tau^{2}}{\sigma^{2}}\left(d^{2}+d \delta\right) .
$$

Put $c_{26}=2 \sqrt{2 \pi} \max \left\{c_{24} e^{100}, c_{25} e^{121}\right\}$. It is clear that choosing $c_{1}, c_{2}$ to be sufficiently small (see $(4.2),(2.21)$ ) we can ensure the validity of the inequality

$$
r=c_{26} \tau^{2} \sigma^{-1}\left(d^{2}+d \delta\right) \leq \sigma .
$$

Hence,

$$
z-r \geq-\sigma \quad \text { for } \quad z \geq 0
$$

Taking into account $(4.55),(4.56)$, choosing $c_{1}, c_{2}, c_{3}$ to be small enough and applying the inequality (2.18) of Lemma 2.14, we see that, for $0 \leq z \leq 10 \sigma$,

$$
\int_{z-r}^{z} v_{1}(y) d y \geq \int_{z-r}^{z} \frac{1}{2 \sqrt{2 \pi} \sigma} e^{-y^{2} / \sigma^{2}} d y \geq \frac{e^{-100} r}{2 \sqrt{2 \pi} \sigma} \geq c_{24} \frac{\tau^{2}}{\sigma^{2}}\left(d^{2}+d \delta\right)
$$

and, analogously,

$$
\int_{z}^{z+r} v_{1}(y) d y \geq \int_{z}^{z+r} \frac{1}{2 \sqrt{2 \pi} \sigma} e^{-y^{2} / \sigma^{2}} d y \geq \frac{e^{-121} r}{2 \sqrt{2 \pi} \sigma} \geq c_{25} \frac{\tau^{2}}{\sigma^{2}}\left(d^{2}+d \delta\right) .
$$

Now from $(4.53),(4.54),(4.57),(4.58)$ it follows that

$$
\int_{z+r}^{\infty} v_{1}(y) d y<\int_{z}^{\infty} v_{2}(y) d y<\int_{z-r}^{\infty} v_{1}(y) d y
$$

for $0 \leq z \leq 10 \sigma$. The statement of Lemma 4.1 for $z \geq 0$ can be easily derived from Lemma 4.3 and from (4.55), (4.59). The case $z \leq 0$ can be considered in a similar way. 


\section{DYADIC SCHEME}

Below we describe an extension of the multivariate version of the KMT dyadic scheme due to Einmahl (1989).

Let $N$ be a positive integer and $\left\{\xi_{1}, \ldots, \xi_{2^{N}}\right\},\left\{Y_{1}, \ldots, Y_{2^{N}}\right\}$ are two collections of independent random vectors. We shall always suppose that the random vectors $\xi_{1}, \ldots, \xi_{2^{N}} ; Y_{1}, \ldots, Y_{2^{N}}$ have some known distributions from $\mathcal{A}_{d}(c \tau)$. Assume that the distributions of $Y_{1}, \ldots, Y_{2^{N}}$ are absolutely continuous. Denote

$$
\begin{gathered}
\widetilde{S}_{0}=0 ; \quad \widetilde{S}_{k}=\sum_{l=1}^{k} \xi_{l}, \quad 1 \leq k \leq 2^{N} ; \\
U_{m, k}^{*}=\widetilde{S}_{(k+1) \cdot 2^{m}}-\widetilde{S}_{k \cdot 2^{m}}, \quad 0 \leq k<2^{N-m}, \quad 0 \leq m \leq N .
\end{gathered}
$$

In particular, $U_{0, k}^{*}=\xi_{k+1}, U_{N, 0}^{*}=\widetilde{S}_{2^{N}}=\xi_{1}+\cdots+\xi_{2^{N}}$. In the sequel we shall use the term block of summands for a collection of summands with indices of the form $k \cdot 2^{m}+1, \ldots,(k+1) \cdot 2^{m}$, where $0 \leq k<2^{N-m}, 0 \leq m \leq N$. Thus, $U_{m, k}^{*}$ is the sum over a block containing $2^{m}$ summands. Put also

$$
\tilde{U}_{n, k}^{*}=U_{n-1,2 k}^{*}-U_{n-1,2 k+1}^{*}, \quad 0 \leq k<2^{N-n}, \quad 1 \leq n \leq N .
$$

Note that

$$
U_{n-1,2 k}^{*}+U_{n-1,2 k+1}^{*}=U_{n, k}^{*}, \quad 0 \leq k<2^{N-n}, \quad 1 \leq n \leq N .
$$

Below we describe a procedure of constructing the random vectors $\left\{U_{n, k}\right\}$ with $\mathcal{L}\left(\left\{U_{n, k}\right\}\right)=\mathcal{L}\left(\left\{U_{n, k}^{*}\right\}\right)$, provided that the vectors $Y_{1}, \ldots, Y_{2^{N}}$ are already constructed. For this purpose we shall use the so-called Rosenblatt quantile transformation (see Rosenblatt (1952) and Einmahl (1989)).

Define the coordinate mappings $\pi_{i}, \widehat{\pi}_{j}, i, j=1, \ldots, d$, by the equalities $\pi_{i}\left(x_{1}, \ldots, x_{d}\right)=x_{i}, \widehat{\pi}_{j}=\left(\pi_{1}, \ldots, \pi_{j}\right)$. Denote by $F_{N, 0}^{(1)}\left(x_{1}\right)=\mathbb{P}\left\{\pi_{1}\left(U_{N, 0}^{*}\right)<\right.$ $\left.x_{1}\right\}, x_{1} \in \mathbb{R}^{1}$, the distribution function of the first coordinate of the vector $U_{N, 0}^{*}$. Let $F_{N, 0}^{(j)}\left(\cdot \mid x_{1}, \ldots, x_{j-1}\right), 2 \leq j \leq d$, be the regular conditional distribution function (r.c.d.f.) of the random variable $\pi_{j}\left(U_{N, 0}^{*}\right)$, given $\widehat{\pi}_{j-1}\left(U_{N, 0}^{*}\right)=\left(x_{1}, \ldots, x_{j-1}\right)$. Furthermore, let $\widetilde{F}_{n, k}^{(1)}\left(\cdot \mid y_{1}, \ldots, y_{d}\right)$ be the r.c.d.f. of $\pi_{1}\left(\widetilde{U}_{n, k}^{*}\right)$, given $U_{n, k}^{*}=\left(y_{1}, \ldots, y_{d}\right)$. Finally, denote by $\widetilde{F}_{n, k}^{(j)}\left(\cdot \mid y_{1}\right.$, $\left.\ldots, y_{d} ; x_{1}, \ldots, x_{j-1}\right)$ the r.c.d.f. of $\pi_{j}\left(\widetilde{U}_{n, k}^{*}\right)$, given $U_{n, k}^{*}=\left(y_{1}, \ldots, y_{d}\right)$ and $\widehat{\pi}_{j-1}\left(\widetilde{U}_{n, k}^{*}\right)=\left(x_{1}, \ldots, x_{j-1}\right), 0 \leq k<2^{N-n}, 1 \leq n \leq N, 2 \leq j \leq d$. Put

$$
\begin{gathered}
T_{0}=0, \quad T_{k}=\sum_{l=1}^{k} Y_{l}, \quad 1 \leq k \leq 2^{N} ; \\
V_{m, k}=\left(V_{m, k}^{(1)}, \ldots, V_{m, k}^{(d)}\right)=T_{(k+1) \cdot 2^{m}}-T_{k \cdot 2^{m}}, \\
0 \leq k<2^{N-m}, \quad 0 \leq m \leq N ; \\
\tilde{V}_{n, k}=\left(\tilde{V}_{n, k}^{(1)}, \ldots, \tilde{V}_{n, k}^{(d)}\right)=V_{n-1,2 k}-V_{n-1,2 k+1}, \\
0 \leq k<2^{N-n}, \quad 1 \leq n \leq N .
\end{gathered}
$$


For the r.c.d.f. of these vectors we shall use the similar notation. Denote by $G_{N, 0}^{(1)}\left(x_{1}\right)=\mathbb{P}\left\{\pi_{1}\left(V_{N, 0}\right)<x_{1}\right\}, x_{1} \in \mathbb{R}^{1}$, the distribution function of the first coordinate of the vector $V_{N, 0}$. Let $G_{N, 0}^{(j)}\left(\cdot \mid x_{1}, \ldots, x_{j-1}\right)$ be the r.c.d.f. of $\pi_{j}\left(V_{N, 0}\right)$, given $\widehat{\pi}_{j-1}\left(V_{N, 0}\right)=\left(x_{1}, \ldots, x_{j-1}\right), 2 \leq j \leq d$. Let $\widetilde{G}_{n, k}^{(1)}\left(\cdot \mid y_{1}, \ldots, y_{d}\right)$ be the r.c.d.f. of $\pi_{1}\left(\widetilde{V}_{n, k}\right)$, given $V_{n, k}=\left(y_{1}, \ldots, y_{d}\right)$, and $\widetilde{G}_{n, k}^{(j)}\left(\cdot \mid y_{1}, \ldots, y_{d} ; x_{1}, \ldots, x_{j-1}\right)$ the r.c.d.f. of $\pi_{j}\left(\widetilde{V}_{n, k}\right)$, given $V_{n, k}=$ $\left(y_{1}, \ldots, y_{d}\right)$ and $\widehat{\pi}_{j-1}\left(\tilde{V}_{n, k}\right)=\left(x_{1}, \ldots, x_{j-1}\right), 0 \leq k<2^{N-n}, 1 \leq n \leq N$, $2 \leq j \leq d$.

Denote now the new collection of random vectors $X_{k}$ as follows. At first we define

$$
\begin{aligned}
& U_{N, 0}^{(1)}=\left(F_{N, 0}^{(1)}\right)^{-1}\left(G_{N, 0}^{(1)}\left(V_{N, 0}^{(1)}\right)\right) \quad \text { and, } \quad \text { for } \quad 2 \leq j \leq d \\
& U_{N, 0}^{(j)}=\left(F_{N, 0}^{(j)}\right)^{-1}\left(G_{N, 0}^{(j)}\left(V_{N, 0}^{(j)} \mid V_{N, 0}^{(1)}, \ldots, V_{N, 0}^{(j-1)}\right) \mid U_{N, 0}^{(1)}, \ldots, U_{N, 0}^{(j-1)}\right)
\end{aligned}
$$

(here $\left(F_{N, 0}^{(1)}\right)^{-1}(t)=\sup \left\{x: F_{N, 0}^{(1)}(x) \leq t\right\}, 0<t<1$, and so on). If the distributions of the random vectors $\xi_{1}, \ldots, \xi_{2^{N}}$ are absolutely continuous, then this formula can be rewritten in a more natural "symmetric" form (see Sakhanenko (1984), pp. 30-31):

$$
\begin{aligned}
F_{N, 0}^{(1)}\left(U_{N, 0}^{(1)}\right) & =G_{N, 0}^{(1)}\left(V_{N, 0}^{(1)}\right) \quad \text { and, for } \quad 2 \leq j \leq d, \\
F_{N, 0}^{(j)}\left(U_{N, 0}^{(j)} \mid U_{N, 0}^{(1)}, \ldots, U_{N, 0}^{(j-1)}\right) & =G_{N, 0}^{(j)}\left(V_{N, 0}^{(j)} \mid V_{N, 0}^{(1)}, \ldots, V_{N, 0}^{(j-1)}\right) .
\end{aligned}
$$

Assume that the random vectors $U_{n, k}=\left(U_{n, k}^{(1)}, \ldots, U_{n, k}^{(d)}\right), 0 \leq k<2^{N-n}$, $1 \leq n \leq N$, corresponding to blocks containing each $2^{n}$ summands, are already constructed. Now we want to pass to the sums containing each $2^{n-1}$ summands. For this for $0 \leq k<2^{N-n}$ we define

$$
\widetilde{U}_{n, k}^{(1)}=\left(\widetilde{F}_{n, k}^{(1)}\right)^{-1}\left(\widetilde{G}_{n, k}^{(1)}\left(\widetilde{V}_{n, k}^{(1)} \mid V_{n, k}\right) \mid U_{n, k}\right),
$$

and, for $2 \leq j \leq d$,

$$
\widetilde{U}_{n, k}^{(j)}=\left(\widetilde{F}_{n, k}^{(j)}\right)^{-1}\left(\widetilde{G}_{n, k}^{(j)}\left(\widetilde{V}_{n, k}^{(j)} \mid V_{n, k} ; \widetilde{V}_{n, k}^{(1)}, \ldots, \widetilde{V}_{n, k}^{(j-1)}\right) \mid U_{n, k} ; \widetilde{U}_{n, k}^{(1)}, \ldots, \widetilde{U}_{n, k}^{(j-1)}\right) .
$$

It is clear that we can rewrite the relations $(5.8),(5.9)$ in the symmetric form, similar to $(5.7)$. Then we put $\widetilde{U}_{n, k}=\left(\widetilde{U}_{n, k}^{(1)}, \ldots, \widetilde{U}_{n, k}^{(d)}\right)$,

$$
\begin{aligned}
U_{n-1,2 k} & =\frac{1}{2}\left(U_{n, k}+\widetilde{U}_{n, k}\right), \\
U_{n-1,2 k+1} & =\frac{1}{2}\left(U_{n, k}-\widetilde{U}_{n, k}\right) .
\end{aligned}
$$

Thus, we have constructed the random vectors $U_{n-1, k}, 0 \leq k<2^{N-n+1}$. After $N$ steps we shall obtain the random vectors $U_{0, k}, 0 \leq k<2^{N}$. Now we set

$$
X_{k}=U_{0, k-1}, \quad S_{0}=0, \quad S_{k}=\sum_{l=1}^{k} X_{l}, \quad 1 \leq k \leq 2^{N} .
$$


One can verify that the mutual distribution of the constructed vectors $U_{n, k}$ coincides with the mutual distribution of the vectors $U_{n, k}^{*}$. In particular, the vectors $X_{k}, k=1, \ldots, 2^{N}$, are independent with $\mathcal{L}\left(X_{k}\right)=\mathcal{L}\left(\xi_{k}\right)$. Moreover, according to $(5.3),(5.4)$, we have

$$
\begin{aligned}
& \widetilde{U}_{n, k}=U_{n-1,2 k}-U_{n-1,2 k+1}, \\
& U_{n, k}=U_{n-1,2 k}+U_{n-1,2 k+1},
\end{aligned} \quad 0 \leq k<2^{N-n}, \quad 1 \leq n \leq N
$$

(it is clear, that (5.12) follows from (5.10)).

The scheme described above is somewhat more general in comparison with that from Einmahl (1989). In Einmahl (1989) it was supposed that the vectors $Y_{k}$ have independent coordinates. However, the fact that our scheme gives the vectors with needed mutual distributions can be justified with the help of KMT (1975-76, p. 117), Sakhanenko (1984, pp. 30-33) and Einmahl (1989, Theorem 6). For this we note that we can begin with independent Gaussian vectors $\left\{Z_{1}, \ldots, Z_{2^{N}}\right\}$ with $\mathcal{L}\left(Z_{k}\right)=\mathcal{N}(0, I)$ and construct the vectors $\left\{X_{1}, \ldots, X_{2^{N}}\right\}$ and $\left\{Y_{1}, \ldots, Y_{2^{N}}\right\}$ as the functions of vectors $\left\{Z_{1}, \ldots, Z_{2^{N}}\right\}$ using our scheme. The vectors $Z_{k}$ have independent coordinates and the vectors $\left\{X_{1}, \ldots, X_{2^{N}}\right\}$ and $\left\{Y_{1}, \ldots, Y_{2^{N}}\right\}$ are connected by the relations (5.5)-(5.11). This is especially clear for the relations (5.7). Now we can exclude the vectors $\left\{Z_{1}, \ldots, Z_{2^{N}}\right\}$ from the scheme.

Note that we essentially use here the assumption about the absolute continuity of the distributions of $Y_{1}, \ldots, Y_{2}$. This assumption implies the absolute continuity of the distributions of the vectors $V_{n, k}$ and, hence, the same property of the projections $\widehat{\pi}_{j}\left(V_{n, k}\right)$. In this case the regular conditional distributions can be defined via conditional densities. Therefore, with probability one, the r.c.d.f. $G$ and $\widetilde{G}$ involved in the relations $(5.7)-(5.9)$ are continuous.

LeMma 5.1. Let $s, r$ be non-negative integers and $1 \leq m=(2 s+1) \cdot 2^{r} \leq 2^{N}$. Then

$$
S_{m}=\frac{m}{2^{N}} S_{2^{N}}+\sum_{n=r+1}^{N} \gamma_{n} \widetilde{U}_{n, l_{n, m}},
$$

where $\gamma_{n}=\gamma_{n}(m) \in[0,1]$ and the integers $l_{n, m}$ are defined by

$$
l_{n, m} \cdot 2^{n}<m \leq\left(l_{n, m}+1\right) \cdot 2^{n} .
$$

Remark 5.2. Lemma 5.1 is contained in Einmahl (1986, Lemma 5, p. 47) or $(1989$, Lemma 5, p. 55). U. Einmahl informed the author that the shortest proof of Lemma 5.1 can be obtained with the help of a geometrical approach due to Massart (1989, p. 275).

Corollary 5.3. Under the conditions of Lemma 5.1

$\left|S_{m}-T_{m}\right| \leq\left|U_{N, 0}-V_{N, 0}\right|+\sum_{n=r+1}^{N}\left|\widetilde{U}_{n, l_{n, m}}-\tilde{V}_{n, l_{n, m}}\right|, \quad m=1, \ldots, 2^{N}$.

This assertion evidently follows from Lemma 5.1 and from the relations (5.1)-(5.6) 
Remark 5.4. Applying Lemma 5.1 and Corollary 5.3, one should remember that $U_{n, l_{n, m}}$ is the sum over the block of $2^{n}$ summands which contains $X_{m}$, the last summand in the sum $S_{m}$.

\section{Estimation OF THE RATE OF APPROXIMATION IN CONSTRUCTING THE VECTORS WITH NEEDED MOMENTS UP TO THE THIRD ORDER}

Below we shall need the following useful fact.

Lemma 6.1. Assume that $0<\alpha<1$ and a random variable $\mu$ has values 0 with probability $1-\alpha^{3}$ and $\alpha^{-1}$ with probability $\alpha^{3}$. Let $X, K \in \mathbb{R}^{d}$ be random vectors with $\mathbb{E} X=\mathbb{E} K=0$, cov $X=D$, cov $K=(1-\alpha) D$, $\mathbb{E}\|X\|^{3}<\infty, \mathbb{E}\|K\|^{3}<\infty$. Suppose also that $X, K, \mu$ are mutually independent and the distribution of $K$ is symmetric. Then the random vector $W=\mu X+K$ has mean zero, covariance operator $D$ and, for any $u, v, w \in \mathbb{R}^{d}$, we have

$$
\mathbb{E}\langle W, u\rangle\langle W, v\rangle\langle W, w\rangle=\mathbb{E}\langle X, u\rangle\langle X, v\rangle\langle X, w\rangle
$$

(in other words, $W$ and $X$ have the same moments of the first three orders). The proof of Lemma 6.1 is elementary and, therefore, is omitted. One should note that Lemma 6.1 is a simplified (and modified) version of (due to Yurinskii (1978)) Lemma 4 from Sazonov (1981, p. 85).

Consider a one-dimensional symmetric probability distribution $H=\mathcal{L}(\eta)$ concentrated on the interval $[-1,1]$ and having an everywhere infinitely differentiable density $w(x)$. Suppose that $\mathbb{E} \eta^{2}=1 / 2$. It is clear that $\mathbb{E} \eta=0$ and such a distribution can be easily constructed. Below we shall assume that the distribution $H$ with properties indicated above is fixed. Consider a two-dimensional random vector $\eta^{*}=\left(\eta_{1}^{*}, \eta_{2}^{*}\right)$ having independent coordinates $\eta_{j}^{*}$, with $\mathcal{L}\left(\eta_{j}^{*}\right)=H, j=1,2$. Define $\zeta=\left(\eta_{1}^{*}+\eta_{2}^{*}, \eta_{1}^{*}-\eta_{2}^{*}\right), H^{*}=\mathcal{L}(\zeta)$. Denote by $H_{h}=\bar{H}(h) \in \mathcal{F}_{1}, h \in \mathbb{R}^{1} ; H_{h}^{*}=\bar{H}^{*}(h) \in \mathcal{F}_{2}, h \in \mathbb{R}^{2}$, the corresponding conjugate distributions.

Lemma 6.2. There exist absolute positive constants $c_{1}, \ldots, c_{10}$ such that the following relations are true:

$$
\begin{gathered}
\sup _{|h| \leq c_{1}} \sup _{|t| \geq c_{2}}\left|\widehat{H}_{h}(t)\right| \leq c_{3}<1, \\
\sup _{|h| \leq c_{1}}\left|\widehat{H}_{h}(t)\right| \leq \exp \left(-\frac{1}{6} t^{2}\right) \quad \text { for } \quad|t| \leq c_{2}, \\
\sup _{|h| \leq 1} \int_{\mathbb{R}^{1}}\left|\widehat{H}_{h}(t)\right| d t \leq c_{4}, \quad \sup _{|h| \leq 1} \int t_{\mathbb{R}^{1}}^{2}\left|\widehat{H}_{h}(t)\right| d t \leq c_{5}, \\
\sup _{\|h\| \leq c_{6}} \sup _{|t| \geq c_{7}}\left|\widehat{H}_{h}^{*}(t)\right| \leq c_{8}<1, \\
\sup _{\|h\| \leq c_{6}}\left|\widehat{H}_{h}^{*}(t)\right| \leq \exp \left(-\frac{1}{3}\|t\|^{2}\right) \quad \text { for } \quad|t| \leq c_{7}, \\
\sup _{\|h\| \leq 1} \int_{\mathbb{R}^{2}}\left|\widehat{H}_{h}^{*}(t)\right| d t \leq c_{9}, \quad \sup _{\|h\| \leq 1} \int\|t\|_{\mathbb{R}^{2}}^{2}\left|\widehat{H}_{h}^{*}(t)\right| d t \leq c_{10} .
\end{gathered}
$$


Proof. We prove the relations (6.5)-(6.7) only. The inequalities (6.2)-(6.4) can be proved analogously. Denote by $w^{*}(x), x \in \mathbb{R}^{2}$, the density of the distribution $H^{*}$. Putting $w^{*}(h, x)=e^{\langle h, x\rangle} w^{*}(x), h, x \in \mathbb{R}^{2}$, using (2.14) and integrating by parts, we see that (for $t=\left(t_{1}, t_{2}\right) \in \mathbb{R}^{2}, t_{j} \neq 0, j=1,2$ )

$$
\begin{aligned}
\widehat{H}_{h}^{*}(t) & =\left(\mathbb{E} e^{\langle h, \zeta\rangle}\right)^{-1} \int_{|x| \leq \sqrt{2}} e^{i\langle t, x\rangle} w^{*}(h, x) d x \\
& =\left(\mathbb{E} e^{\langle h, \zeta\rangle}\right)^{-1} \int_{|x| \leq \sqrt{2}} t_{1}^{-4} t_{2}^{-4} e^{i\langle t, x\rangle} \frac{\partial^{8} w^{*}(h, x)}{\partial x_{1}^{4} \partial x_{2}^{4}} d x .
\end{aligned}
$$

Moreover,

$$
\sup _{x \in \mathbb{R}^{2}} \sup _{\|h\| \leq 1}\left|\frac{\partial^{8} w^{*}(h, x)}{\partial x_{1}^{4} \partial x_{2}^{4}}\right| d x \leq c_{11}
$$

(constants here and below are absolute, since the distribution $H^{*}$ is fixed). From (6.8), (6.9) it follows that

$$
\sup _{\|h\| \leq 1}\left|\hat{H}_{h}^{*}(t)\right| \leq 2 c_{11} t_{1}^{-4} t_{2}^{-4}
$$

(we take into account that, by the Jensen inequality, $\mathbb{E} e^{\langle h, \zeta\rangle} \geq e^{\mathbb{E}\langle h, \zeta\rangle}=1$ ). It is evident that with the help of analogous integrations by parts (different for different values of $t$ ) one can prove that

$$
\sup _{\|h\| \leq 1}\left|\widehat{H}_{h}^{*}(t)\right| \leq \frac{c_{12}}{\left(1+t_{1}^{4}\right)\left(1+t_{2}^{4}\right)} .
$$

Hence,

$$
\sup _{\|h\| \leq 1} \sup _{|t| \geq c_{13}}\left|\widehat{H}_{h}^{*}(t)\right| \leq 1 / 2
$$

(one can take $\left.c_{13}=\left(2 c_{12}\right)^{1 / 4}\right)$. Inequalities (6.7) follow from (6.11).

Obviously, $H^{*} \in \mathcal{A}_{2}(c)$. It is easy to see that $\operatorname{cov} H^{*}=I: \mathbb{R}^{2} \rightarrow \mathbb{R}^{2}$. Using Lemma 2.4(b), (2.4) and (2.6), we see that the relation (6.6) is valid, if $c_{6} \leq 1$ and $c_{7} \leq c_{13}$ are sufficiently small.

It is clear that the distributions $H_{h}^{*}$ are absolutely continuous. This implies that the relation $\left|\widehat{H}_{h}^{*}(t)\right|=1$ can be valid for $t=0$ only. Moreover, the function $\left|\widehat{H}_{h}^{*}(t)\right|$ considered as function of two variables $h$ and $t$ is continuous for all $h, t \in \mathbb{R}^{2}$. Therefore,

$$
\sup _{\|h\| \leq c_{6} c_{7} \leq|t| \leq c_{13}} \sup _{h}\left|\widehat{H}_{h}^{*}(t)\right| \leq c<1
$$

The inequalities $(6.12),(6.13)$ imply (6.5).

Definition 6.3. Assume that the conditions of Lemma 6.1 are satisfied with $D=I, \alpha=1 / 2$, and the vector $K$ has independent coordinates $K_{j}$ with $\mathcal{L}\left(K_{j}\right)=H, j=1, \ldots, d$. In this case (it is evident that $\mathbb{E} K=0$, $\operatorname{cov} K=I / 2)$ we shall use below the notation $\Psi(\mathcal{L}(X))=\mathcal{L}(W)$. 
Suppose that $\mathcal{L}(X) \in \mathcal{A}_{d}(\tau), \tau \geq 1$. It is clear that the distribution $\Psi(\mathcal{L}(X))$ is always absolutely continuous with infinitely differentiable density. Using Lemma 2.7, Remark 2.9, the completeness of classes $\mathcal{A}_{d}(\tau)$ with respect to convolution and their monotonicity with respect to $\tau$, one can show that there exists $c_{14}, c_{15}$ such that

$$
\mathcal{L}(K) \in \mathcal{A}_{d}\left(c_{14}\right), \quad \mathcal{L}(W)=\Psi(\mathcal{L}(X)) \in \mathcal{A}_{d}\left(c_{15} \tau\right), \quad c_{15} \geq 1 .
$$

The simplest way to prove the second relation in $(6.14)$ is to use a connection between classes $\mathcal{A}_{d}(\tau)$ and classes of distributions satisfying Bernstein-type inequality conditions (see Zaitsev (1986, p. 205; 1987, 1988)).

Let $\tau \geq 1$ and $\widetilde{\xi}_{1}, \ldots, \widetilde{\xi}_{2^{N}}$, be independent random vectors with known distributions such that $\mathcal{L}\left(\widetilde{\xi}_{k}\right) \in \mathcal{A}_{d}(\tau), \mathbb{E} \widetilde{\xi}_{k}=0, \operatorname{cov} \widetilde{\xi}_{k}=I, k=1, \ldots, 2^{N}$. Assume that we have the independent random vectors $\xi_{1}, \ldots, \xi_{2 N}$ with $\mathcal{L}\left(\xi_{k}\right)=\Psi\left(\mathcal{L}\left(\widetilde{\xi}_{k}\right)\right), k=1, \ldots, 2^{N}$. According to Lemma $6.1, \xi_{k}$ and $\widetilde{\xi}_{k}$ have the same moments of the first three orders. Let $Y_{1}, \ldots, Y_{2^{N}}$ be independent Gaussian random vectors with $\mathcal{L}\left(Y_{k}\right)=\Phi\left(\mathcal{L}\left(\widetilde{\xi}_{k}\right)\right)$. Assume that the random vectors $X_{k}, \mathcal{L}\left(X_{k}\right)=\mathcal{L}\left(\xi_{k}\right) ; V_{n, k}, \widetilde{V}_{n, k} ; U_{n, k}, \widetilde{U}_{n, k}, S_{k}, T_{k}$ are constructed as functions of the vectors $Y_{1}, \ldots, Y_{2}$ as in Section 5. Taking into account (6.14) and using the completeness of classes $\mathcal{A}_{d}\left(c_{15} \tau\right)$ with respect to convolution, we have

$$
\begin{gathered}
\mathcal{L}\left(X_{k}\right)=\mathcal{L}\left(\xi_{k}\right) \in \mathcal{A}_{d}\left(c_{15} \tau\right), \quad k=1, \ldots, 2^{N} ; \\
\mathcal{L}\left(U_{n, k}\right) \in \mathcal{A}_{d}\left(c_{15} \tau\right), \quad 0 \leq k<2^{N-n}, \quad 0 \leq n \leq N .
\end{gathered}
$$

THEOREM 6.4. Let the conditions described above be satisfied with $N \geq 1$. Then there exist absolute positive constants $c_{16}, c_{17}$ such that

$$
\mathbb{E} \exp \left(\frac{c_{16} \Delta}{d^{3 / 2} \tau}\right) \leq \exp \left(c_{17} N L(d)\right),
$$

where

$$
\Delta=\Delta(X, Y)=\max _{1 \leq i \leq 2^{N}}\left|S_{i}-T_{i}\right| .
$$

The rest of this section is devoted to the proof of Theorem 6.4. Therefore, below we assume that its conditions are fulfilled. The following Lemma 6.5 can be considered as an analog of Lemma 4 from Einmahl's (1989, p. 55).

LEMMA 6.5. There exist absolute positive constants $c_{18}, \ldots, c_{23}$ such that

a) if $\tau d^{3 / 2} / 2^{N / 2} \leq c_{18}$, then

$$
\left|U_{N, 0}-V_{N, 0}\right| \leq c_{19} d^{3 / 2} \tau\left(1+2^{-N}\left|U_{N, 0}\right|^{2}\right)
$$

provided that $\left|U_{N, 0}\right| \leq \frac{c_{20} \cdot 2^{N}}{d^{3 / 2} \tau}$

b) if $1 \leq n \leq N, 0 \leq k<2^{N-n}, \tau d^{3 / 2} / 2^{n / 2} \leq c_{21}$, then

$$
\left|\widetilde{U}_{n, k}-\tilde{V}_{n, k}\right| \leq c_{22} d^{3 / 2} \tau\left(1+2^{-n} \max \left\{\left|U_{n, k}\right|^{2},\left|\widetilde{U}_{n, k}\right|^{2}\right\}\right)
$$

provided that $\max \left\{\left|U_{n, k}\right|,\left|\widetilde{U}_{n, k}\right|\right\} \leq \frac{c_{23} \cdot 2^{n}}{d^{3 / 2} \tau}$. 
Proof. At first we prove assertion a). From the properties of classes $\mathcal{A}_{d}(\tau)$ and from (6.15) it follows that $\mathcal{L}\left(U_{N, 0}\right) \in \mathcal{A}_{d}\left(c_{15} \tau\right), \mathcal{L}\left(V_{N, 0}\right)=\Phi\left(\mathcal{L}\left(U_{N, 0}\right)\right)$. Below we prove that if $c_{18}$ is small enough, then these distributions satisfy the conditions of Lemmas 2.14 and 3.1 with $D=2^{N} I, \sigma^{2}=2^{N}$ and with the change of $\tau$ by $c_{24} \tau$, where $c_{24}=\max \left\{c_{15}, c_{1}^{-1}, 1\right\}$. For this we shall verify that for $h \in \mathbb{R}^{d},\|h\| c_{24} \tau<1$, and for all $v \in \mathbb{R}^{d}$ the following inequalities hold (see (2.15) and Definition 2.12):

$$
\begin{gathered}
(2 \pi)^{-d} \int_{T}\left|\widehat{F}_{h}(t)\right| d t \leq \frac{c_{24}^{2} \tau^{2} d^{2}}{2^{N}(2 \pi)^{d / 2} \cdot 2^{N d / 2}}, \\
(2 \pi)^{-d} \int_{T}\left|\langle t, v\rangle \widehat{F}_{h}(t)\right| d t \leq \frac{\|v\|}{4 \cdot 2^{N / 2}(2 \pi)^{d / 2} \cdot 2^{N d / 2}},
\end{gathered}
$$

where $F_{h}=\bar{F}(h), F=\mathcal{L}\left(U_{N, 0}\right)$,

$$
T=\left\{t \in \mathbb{R}^{d}: 4\|t\| c_{24} \tau d \geq 1\right\} .
$$

Besides, we need the validity of analogous relations in the $(d-1)$-dimensional situation for the distributions $F^{\prime}=\mathcal{L}\left(U_{N, 0}^{\prime}\right)$ (see Notation 2.13).

From the definition of distributions $\Psi(\cdot)$ and from (2.2) it follows that

$$
\left|\widehat{F}_{h}(t)\right| \leq \prod_{j=1}^{d}\left|\widehat{H}_{h_{j}}\left(t_{j}\right)\right|^{2^{N}}
$$

Since $c_{24} \geq c_{1}^{-1}, \tau \geq 1$, we can use for estimating $\left|\widehat{H}_{h_{j}}\left(t_{j}\right)\right|$ the inequalities $(6.2)-(6.4)$. In particular, from $(6.2),(6.3),(6.21)$ one can easily derive that

$$
\sup _{t \in T} \prod_{j=1}^{d}\left|\widehat{H}_{h_{j}}\left(t_{j}\right)\right| \leq \max \left\{\exp \left(-\left(96 c_{24}^{2} \tau^{2} d^{2}\right)^{-1}\right), c_{3}\right\} \leq \exp \left(-\frac{c_{25}}{\tau^{2} d^{2}}\right) .
$$

From $(6.22),(6.23)$ it follows that

$$
\left|\widehat{F}_{h}(t)\right| \leq \exp \left(-\frac{2^{N} c_{25}}{2 \tau^{2} d^{2}}\right) \prod_{j=1}^{d}\left|\widehat{H}_{h_{j}}\left(t_{j}\right)\right|^{2^{N-1}}, \quad t \in T .
$$

Choosing $c_{18}$ to be sufficiently small and taking into account that $\tau \geq 1$, we ensure the validity of the inequality

$$
2^{N-1} \geq 2
$$

Using (6.3), we obtain

$$
\int_{\left|t_{j}\right| \leq c_{2}}\left|\widehat{H}_{h_{j}}\left(t_{j}\right)\right|^{2^{N-1}} d t_{j} \leq \int_{|t| \leq c_{2}} \exp \left(-\frac{2^{N} t^{2}}{12}\right) d t \leq \frac{c}{2^{N / 2}} .
$$


Analogously, using (6.2), (6.4), (6.25), we have

$$
\int_{\left|t_{j}\right|>c_{2}}\left|\hat{H}_{h_{j}}\left(t_{j}\right)\right|^{2^{N-1}} d t_{j} \leq \int_{|t|>c_{2}} c_{3}^{2^{N-2}}\left|\widehat{H}_{h_{j}}(t)\right| d t \leq c \exp \left(-c \cdot 2^{N}\right) \leq \frac{c}{2^{N / 2}} .
$$

From $(6.26),(6.27)$ it follows that

$$
\int_{\mathbb{R}^{1}}\left|\widehat{H}_{h_{j}}\left(t_{j}\right)\right|^{2^{N-1}} d t_{j} \leq \frac{c}{2^{N / 2}} .
$$

Using now (6.24), (6.28), we see that

$$
(2 \pi)^{-d} \int_{T}\left|\widehat{F}_{h}(t)\right| d t \leq \frac{1}{2^{N d / 2}(2 \pi)^{d / 2}} \exp \left(c_{26} d-\frac{c_{27} \cdot 2^{N}}{\tau^{2} d^{2}}\right) .
$$

It is evident that, choosing $c_{18}$ to be small enough, we can ensure the validity of the inequality

$$
\exp \left(c_{26} d-\frac{c_{27} \cdot 2^{N}}{\tau^{2} d^{2}}\right) \leq \frac{c_{24}^{2} \tau^{2} d^{2}}{2^{N}} \leq \frac{1}{4} .
$$

The inequalities (6.29), (6.30) imply (6.19).

For the proof of $(6.20)$ we apply the Hölder inequality and obtain that

$$
\int_{T}\left|\langle t, v\rangle \widehat{F}_{h}(t)\right| d t \leq\|v\|\left(\int_{T}\left|\widehat{F}_{h}(t)\right| d t\right)^{1 / 2}\left(\int_{T}\|t\|^{2}\left|\widehat{F}_{h}(t)\right| d t\right)^{1 / 2} .
$$

For estimating the first integral in the right-hand side of (6.31) we note that, according to $(6.29),(6.30)$,

$$
(2 \pi)^{-d} \int_{T}\left|\widehat{F}_{h}(t)\right| d t \leq \frac{1}{4(2 \pi)^{d / 2} \cdot 2^{N d / 2}} .
$$

Let us estimate the second integral. For this, using (6.3), we obtain

$$
\int_{\left|t_{j}\right| \leq c_{2}} t_{j}^{2}\left|\widehat{H}_{h_{j}}\left(t_{j}\right)\right|^{2^{N-1}} d t_{j} \leq \int_{|t| \leq c_{2}} t^{2} \exp \left(-\frac{2^{N} t^{2}}{12}\right) d t \leq \frac{c}{2^{3 N / 2}} .
$$

Moreover, using (6.2), (6.4), (6.25), we have

$$
\begin{aligned}
\int_{\left|t_{j}\right|>c_{2}} t_{j}^{2}\left|\widehat{H}_{h_{j}}\left(t_{j}\right)\right|^{2^{N-1}} d t_{j} & \leq \int_{|t|>c_{2}} c_{3}^{2^{N-2}} t^{2}\left|\widehat{H}_{h_{j}}(t)\right| d t \\
& \leq c \exp \left(-c \cdot 2^{N}\right) \leq \frac{c}{2^{3 N / 2}} .
\end{aligned}
$$

From $(6.33),(6.34)$ it follows that

$$
\int_{\mathbb{R}^{1}} t_{j}^{2}\left|\widehat{H}_{h_{j}}\left(t_{j}\right)\right|^{2^{N-1}} d t_{j} \leq \frac{c}{2^{3 N / 2}} .
$$


Using now $(6.24),(6.28),(6.35)$, we see that

$$
(2 \pi)^{-d} \int_{T}\|t\|^{2}\left|\widehat{F}_{h}(t)\right| d t \leq \frac{1}{2^{N}(2 \pi)^{d / 2} \cdot 2^{N d / 2}} \exp \left(c_{28} d-\frac{c_{29} \cdot 2^{N}}{\tau^{2} d^{2}}\right) .
$$

It is evident that, choosing $c_{18}$ to be small enough, we can ensure the validity of the inequality

$$
\exp \left(c_{28} d-\frac{c_{29} \cdot 2^{N}}{\tau^{2} d^{2}}\right) \leq \frac{1}{4} .
$$

The inequalities (6.31), (6.32), (6.36), (6.37) imply (6.20).

The analogs of the relations $(6.19),(6.20)$ for the distributions $F^{\prime}$ follow, in fact, from $(6.19),(6.20)$ applied in the $(d-1)$-dimensional case.

Using Lemma 2.5, we can analogously prove that the conditions of Lemmas 2.14 and 3.1 with $D=2^{N} I, \sigma^{2}=2^{N}$ and with the change of $\tau$ by $c_{24} \tau$ are satisfied in the $j$-dimensional situation for the distributions $\mathcal{L}\left(U_{N, 0}^{j}\right), \mathcal{L}\left(V_{N, 0}^{j}\right), j=1, \ldots, d$, where the vectors

$$
U_{N, 0}^{j}=\left(U_{N, 0}^{(1)}, \ldots, U_{N, 0}^{(j)}\right), \quad V_{N, 0}^{j}=\left(V_{N, 0}^{(1)}, \ldots, V_{N, 0}^{(j)}\right)
$$

consist of the first $j$ coordinates of the vectors $U_{N, 0}, V_{N, 0}$ respectively.

Taking into account (5.7), noting that all distribution functions $G_{N, 0}^{(j)}$ coincide with $\Phi_{\sigma}(\cdot)$ and applying Lemma 3.1 and the inequality (2.16) of Lemma 2.14 in the $j$-dimensional case, $j=1, \ldots, d$, we obtain that

$$
\left|U_{N, 0}^{(1)}-V_{N, 0}^{(1)}\right| \leq c_{30} \tau\left(1+\frac{\left|U_{N, 0}^{(1)}\right|^{2}}{2^{N}}\right)
$$

if $\frac{\tau}{2^{N / 2}} \leq c_{31},\left|U_{N, 0}^{(1)}\right| \leq \frac{c_{32} \cdot 2^{N}}{\tau}$

$$
\begin{aligned}
\left|U_{N, 0}^{(j)}-V_{N, 0}^{(j)}\right| \leq c_{30} \tau\left(j^{3 / 2}+j^{3 / 2} \frac{\left|U_{N, 0}^{j-1}\right|}{2^{N / 2}}\left(1+\frac{\left|U_{N, 0}^{(j)}\right|}{2^{N / 2}}\right)\right. & \\
& \left.+\frac{\left|U_{N, 0}^{(j)}\right|^{2}}{2^{N}}+j \frac{\left|U_{N, 0}^{j-1}\right|^{2}}{2^{N}}\right)
\end{aligned}
$$

if

$$
\frac{\tau j^{3 / 2}}{2^{N / 2}} \leq c_{31}, \quad\left|U_{N, 0}^{(j)}\right| \leq \frac{c_{32} \cdot 2^{N}}{j \tau}, \quad \frac{\left|U_{N, 0}^{j-1}\right|}{2^{N / 2}} \leq \frac{c_{33} \cdot 2^{N / 2}}{j^{3 / 2} \tau}, \quad 2 \leq j \leq d .
$$

Checking the inequality (6.40), one should take into account that in Lemmas 2.14 and 3.1 the densities $v(\cdot)$ are the densities of shifted conditional distributions. The possibility to write the inequality $(6.40)$ and the conditions (6.41) without shift is ensured by a suitable choice of sufficiently small $c_{32}$ and $c_{33}$ with the use of inequality (2.16). Inequality (6.17) with $c_{18} \leq c_{31}, c_{19}=4 c_{30}, c_{20}=\min \left\{c_{32}, c_{33}\right\}$ follows from $(6.39),(6.40)$ and from the definitions of $U_{N, 0}, U_{N, 0}^{j}$. 
Let us prove assertion b). Let $L_{n, k}^{*}=\left(U_{n-1,2 k}, U_{n-1,2 k+1}\right) \in \mathbb{R}^{2 d}$ be the vector with first $d$ coordinates coinciding with those of $U_{n-1,2 k}$ and with last $d$ coordinates coinciding with those of $U_{n-1,2 k+1}$. Denote by $L_{n, k}=$ $\left(U_{n, k}, \widetilde{U}_{n, k}\right) \in \mathbb{R}^{2 d}$ the vector constructed analogously from the coordinates of the vectors $U_{n, k}, \widetilde{U}_{n, k}$. It is evident that the vectors $U_{n-1,2 k}$ and $U_{n-1,2 k+1}$ are independent. Moreover, according to (6.15), the distributions of these vectors belong to $\mathcal{A}_{d}\left(c_{15} \tau\right)$. From Lemma 2.7 it follows that $\mathcal{L}\left(L_{n, k}^{*}\right) \in$ $\mathcal{A}_{2 d}\left(c_{15} \tau\right)$. The relations (5.12) show that $L_{n, k}=A L_{n, k}^{*}$, where $A: \mathbb{R}^{2 d} \rightarrow$ $\mathbb{R}^{2 d}$ is a linear operator with $\|A\|=\sqrt{2}$. Therefore, using Lemma 2.5, we see that $\mathcal{L}\left(L_{n, k}\right) \in \mathcal{A}_{2 d}\left(c_{15} \sqrt{2} \tau\right)$.

Consider as well the Gaussian random vectors $R_{n, k}=\left(V_{n, k}, \widetilde{V}_{n, k}\right) \in \mathbb{R}^{2 d}$. It can be easily verified that (see (5.1)-(5.6)) $\mathcal{L}\left(R_{n, k}\right)=\Phi\left(\mathcal{L}\left(L_{n, k}\right)\right)$. Define also the vectors

$$
L_{n, k}^{j}=\left(L_{n, k}^{(1)}, \ldots, L_{n, k}^{(j)}\right), \quad R_{n, k}^{j}=\left(R_{n, k}^{(1)}, \ldots, R_{n, k}^{(j)}\right), \quad j=d, \ldots, 2 d,
$$

consisting of the first $j$ coordinates of the vectors $L_{n, k}, R_{n, k}$ respectively.

One can show that if $c_{21}$ is small enough, then the distributions $\mathcal{L}\left(L_{n, k}^{j}\right)$ and $\mathcal{L}\left(R_{n, k}^{j}\right), j=d+1, \ldots, 2 d$, satisfy in $j$-dimensional situation the conditions of Lemmas 2.14, 3.1 with $D=2^{n} I, \sigma^{2}=2^{n}$ and with the change of $d$ by $j$ and $\tau$ by $c_{34} \tau$, where $c_{34}=\max \left\{c_{15} \sqrt{2}, c_{1}^{-1}, c_{6}^{-1}, 1\right\}$. For this one should fix $j$ and verify that for $h^{*} \in \mathbb{R}^{j},\left\|h^{*}\right\| c_{34} \tau<1$, and for all $v^{*} \in \mathbb{R}^{j}$, the following inequalities hold (see (2.15) and Definition 2.12):

$$
\begin{gathered}
(2 \pi)^{-j} \int_{T}\left|\widehat{F}_{h^{*}}\left(t^{*}\right)\right| d t^{*} \leq \frac{c_{34}^{2} \tau^{2} j^{2}}{2^{n}(2 \pi)^{j / 2} \cdot 2^{n j / 2}}, \\
(2 \pi)^{-j} \int_{T}\left|\left\langle t^{*}, v^{*}\right\rangle \widehat{F}_{h^{*}}\left(t^{*}\right)\right| d t^{*} \leq \frac{\left\|v^{*}\right\|}{4 \cdot 2^{n / 2}(2 \pi)^{j / 2} \cdot 2^{n j / 2}},
\end{gathered}
$$

where $F_{h^{*}}=\bar{F}\left(h^{*}\right), F=\mathcal{L}\left(L_{n, k}^{j}\right), h^{*}=\left(h_{1}^{*}, \ldots, h_{d}^{*}\right) \in \mathbb{R}^{j}$,

$$
\begin{gathered}
T=\left\{t^{*}=\left(t_{1}^{*}, \ldots, t_{d}^{*}\right) \in \mathbb{R}^{j}: 4\left\|t^{*}\right\| c_{34} \tau j \geq 1\right\} ; \\
h_{m}^{*}=\left(h_{m}, h_{d+m}\right) \in \mathbb{R}^{2}, \quad t_{m}^{*}=\left(t_{m}, t_{d+m}\right) \in \mathbb{R}^{2}, \quad m=1, \ldots, j-d ; \\
h_{m}^{*}=h_{m} \in \mathbb{R}^{1}, \quad t_{m}^{*}=t_{m} \in \mathbb{R}^{1}, \quad m=j-d+1, \ldots, d .
\end{gathered}
$$

Besides, we need the validity of analogous relations in the $(j-1)$-dimensional situation for the distributions $F^{\prime}=\mathcal{L}\left(L_{n, k}^{j-1}\right)$. Thus, one should verify the fulfilment of (6.43), (6.44) for $j=d, \ldots, 2 d$.

Using the definition of distributions $\Psi(\cdot)$ and of random vectors $L_{n, k}^{j}$ and the relations $(2.2),(5.1),(5.2),(5.12)$, we obtain that

$$
\left|\widehat{F}_{h^{*}}\left(t^{*}\right)\right| \leq \prod_{m=1}^{j-d}\left|\widehat{H}_{h_{m}^{*}}^{*}\left(t_{m}^{*}\right)\right|^{2^{n-1}} \prod_{m=j-d+1}^{d}\left|\widehat{H}_{h_{m}}\left(t_{m}\right)\right|^{2^{n}} .
$$

By the definition of $c_{34}$, we can use for estimating $\left|\widehat{H}_{h_{m}}\left(t_{m}\right)\right|$ the inequalities (6.2)-(6.4) and for estimating $\left|\widehat{H}_{h_{m}^{*}}^{*}\left(t_{m}^{*}\right)\right|$ the inequalities $(6.5)-(6.7)$. 
The rest of the proof of inequalities $(6.43),(6.44)$ does not differ from the proof of $(6.19),(6.20)$ and therefore is omitted. The only difference is in the use of $(6.5)-(6.7)$ instead of $(6.2)-(6.4)$ and in the change of constants and notation (see also the proof of $(6.43),(6.44)$ in somewhat more complicated situation in Section 7).

Note that all distribution functions $\widetilde{G}_{n, k}^{(j-d)}(\cdot \mid \cdot)$ coincide with $\Phi_{\sigma}(\cdot)$. Therefore, using (5.8), (5.9) and applying Lemma 3.1 and the inequality (2.16) of Lemma 2.14 in the $j$-dimensional case, $j=d+1, \ldots, 2 d$, we obtain that

$$
\begin{aligned}
\left|L_{n, k}^{(j)}-R_{n, k}^{(j)}\right| \leq c_{35} \tau\left(j^{3 / 2}+j^{3 / 2} \frac{\left|L_{n, k}^{j-1}\right|}{2^{n / 2}}\left(1+\frac{\left|L_{n, k}^{(j)}\right|}{2^{n / 2}}\right)\right. \\
\left.+\frac{\left|L_{n, k}^{(j)}\right|^{2}}{2^{n}}+j \frac{\left|L_{n, k}^{j-1}\right|^{2}}{2^{n}}\right),
\end{aligned}
$$

if

$$
\frac{\tau j^{3 / 2}}{2^{n / 2}} \leq c_{36}, \quad\left|L_{n, k}^{(j)}\right| \leq \frac{c_{37} \cdot 2^{n}}{j \tau}, \quad \frac{\left|L_{n, k}^{j-1}\right|}{2^{n / 2}} \leq \frac{c_{38} \cdot 2^{n / 2}}{j^{3 / 2} \tau} .
$$

Checking the inequality (6.46) should be carried out analogously to the proof of (6.40). The possibility to write the inequality (6.46) and the conditions (6.47) without shift is also ensured by a suitable choice of sufficiently small constants $\left(c_{37}\right.$ and $c_{38}$ ) with the use of inequality (2.16).

Inequality (6.18) follows from $(6.42),(6.46)$ and from the definition of $L_{n, k}, R_{n, k}$ provided that we choose $c_{21} \leq 2^{-3 / 2} c_{36}, c_{22}=2^{3 / 2} \cdot 4 c_{35}$ and $c_{23}=2^{-3 / 2} \min \left\{c_{37}, c_{38}\right\}$.

Proof of Theorem 6.4 . Let us prove that there exists a $c_{39}$ such that

$$
\mathbb{P}\{\Delta \geq x\} \leq(15 d)^{N} \exp \left(-\frac{x}{c_{39} d^{3 / 2} \tau}\right), \quad x \geq 0 .
$$

Denote

$$
c_{40}=\min \left\{c_{20}, c_{23}, c_{15}^{-1}\right\}, \quad c_{41}=\frac{4 c_{40}}{\min \left\{c_{18}^{2}, c_{21}^{2}, 2^{-1}\right\}}, \quad y=\frac{c_{40}}{d^{3 / 2} \tau} .
$$

Assume (without loss of generality) that

$$
x \geq c_{41} d^{3 / 2} \tau
$$

and choose the integer $M$ from the condition

$$
x<4 y \cdot 2^{M} \leq 2 x .
$$

Note that $M \geq 1$ since $(6.49),(6.50)$ and $\tau \geq 1$ imply the inequality $x \geq 8 y$. Denote $L=\min \{M, N\}$,

$$
\begin{aligned}
& \Delta_{1}=\max _{0 \leq k<2^{N-M}} \max _{1 \leq s \leq 2^{L}}\left|S_{k \cdot 2^{M}+s}-S_{k \cdot 2^{M}}\right|, \\
& \Delta_{2}=\max _{0 \leq k<2^{N-M}} \max _{1 \leq s \leq 2^{L}}\left|T_{k \cdot 2^{M}+s}-T_{k \cdot 2^{M}}\right|, \\
& \Delta_{3}=\max _{0 \leq k<2^{N-M}}\left|S_{k \cdot 2^{M}}-T_{k \cdot 2^{M}}\right|,
\end{aligned}
$$


where the maximums are taken over integer values of $k$ and $s$. It is evident that

$$
\Delta \leq \Delta_{1}+\Delta_{2}+\Delta_{3}
$$

Define the event

$$
A=\left\{\omega: \max \left\{\Delta_{1}, \Delta_{2}\right\}<x / 4\right\}
$$

(we shall always assume that all considered random vectors are measurable mappings of elementary event $\omega \in \Omega$ ). Recall that all $d$-dimensional Gaussian distributions belong to classes $\mathcal{A}_{d}(\tau)$ for all $\tau \geq 0$. Applying Lemma 2.11 with parameters $\tau^{*}=c_{15} \tau, B^{2}=2^{L} \leq 2^{M}$, and using (6.49), (6.51)-(6.53) and (6.56), we obtain that

$$
\begin{aligned}
1-\mathbb{P}\{A\} & \leq 2^{N} \cdot 4 d \max \left\{\exp \left(-x^{2} / 64 B^{2}\right), \exp \left(-x / 16 \tau^{*}\right)\right\} \\
& \leq 2^{N} \cdot 4 d \exp \left(-\frac{c x}{d^{3 / 2} \tau}\right) .
\end{aligned}
$$

If $M \geq N$ then $\Delta_{3}=0$, and, for $\omega \in A$, we have $\Delta<x / 2$ (see (6.55) and (6.56)). Hence, $\mathbb{P}\{\Delta \geq x\} \leq 1-\mathbb{P}\{A\}$ and one can conclude via $(6.57)$.

Let now $M<N$. Fix some integer $k, 1 \leq k \leq 2^{N-M}$. According to Corollary 5.3, we have

$$
\left|S_{k \cdot 2^{M}}-T_{k \cdot 2 M}\right| \leq\left|U_{N, 0}-V_{N, 0}\right|+\sum_{n=M+1}^{N}\left|\widetilde{U}_{n, l_{n, j}}-\tilde{V}_{n, l_{n, j}}\right|
$$

where $l_{n, j}$ are integers, defined by $l_{n, j} \cdot 2^{n}<j \stackrel{\text { def }}{=} k \cdot 2^{M} \leq\left(l_{n, j}+1\right) \cdot 2^{n}$ (see $(5.13))$.

By virtue of $(6.49)-(6.51)$, we have $c_{41} d^{3 / 2} \tau \leq x \leq \frac{4 c_{40} \cdot 2^{M}}{d^{3 / 2} \tau}$ and, consequently,

$$
\tau \leq \frac{\min \left\{c_{18}, c_{21}\right\} \cdot 2^{M / 2}}{d^{3 / 2}}, \quad \frac{\tau d^{3 / 2}}{2^{M / 2}} \leq \min \left\{c_{18}, c_{21}\right\}
$$

Recall that $U_{n, l}=S_{(l+1) \cdot 2^{n}}-S_{l \cdot 2^{n}}$. According to (6.49), (6.51) and (6.56), for $\omega \in A$ we have

$$
\left|U_{M, l}\right| \leq \Delta_{1}<y \cdot 2^{M} \leq \frac{\min \left\{c_{20}, c_{23}\right\} \cdot 2^{M}}{d^{3 / 2} \tau}, \quad 0 \leq l<2^{N-M}
$$

The relations $(6.59),(6.60)$ allow us to apply Lemma 6.5 for estimating the right-hand side of (6.58). Using exactly the same arguments as in KMT (1975-76, pp. 120-121), we obtain (for $\omega \in A$ ) the bound

$$
\left|S_{k \cdot 2^{M}}-T_{k \cdot 2^{M}}\right| \leq c_{42} d^{3 / 2} \tau\left(N+W_{M, l_{M, j}}+\sum_{n=M}^{N-1} W_{n, \widetilde{l}_{n, j}}\right)
$$


where (for $0 \leq n \leq N, 0 \leq l<2^{N-n}$ )

$$
W_{n, l}=\left\{\begin{array}{cl}
2^{-n}\left|U_{n, l}\right|^{2}, & \text { if }\left|U_{n, l}\right| \leq y \cdot 2^{n}, \\
0, & \text { otherwise. }
\end{array}\right.
$$

and $U_{n, l_{n, j}}, M \leq n \leq N$, is the sum over the block of $2^{n}$ summands which contains the summand $X_{j}$ (see Remark 5.4). The sum $U_{n, \widetilde{l}_{n, j}}$ does not contain $X_{j}$ and $U_{n+1, l_{n+1, j}}=U_{n, l_{n, j}}+U_{n, \tilde{l}_{n, j}}, M \leq n<N$ (see (5.12)). Thus, all summands in the right-hand side of (6.61) are functions of sums of disjoint blocks of independent summands. Therefore, they are themselves independent.

Let us show that

$$
\mathbb{E} \exp \left(t W_{n, l}\right) \leq 2 d+1, \quad \text { for } \quad 0 \leq t \leq \frac{1}{8} .
$$

Indeed, integrating by parts, we obtain

$$
\begin{aligned}
\mathbb{E} \exp \left(t W_{n, l}\right) & =1+\int_{0}^{y^{2} \cdot 2^{n}} t e^{t u} \mathbb{P}\left\{W_{n, l} \geq u\right\} d u \\
& \leq 1+\frac{1}{8} \int_{0}^{y^{2} \cdot 2^{n}} e^{u / 8} \mathbb{P}\left\{\left|U_{n, l}\right| \geq 2^{n / 2} \sqrt{u}\right\} d u .
\end{aligned}
$$

Recall that the sum $U_{n, l}$ contains $2^{n}$ independent summands $X_{i}$. Hence, using Lemma $2.11,(6.15)$ and (6.49), we get

$$
\mathbb{P}\left\{\left|U_{n, l}\right| \geq 2^{n / 2} \sqrt{u}\right\} \leq 2 d \exp \left(-\min \left\{\frac{2^{n} u}{4 \cdot 2^{n}}, \frac{2^{n / 2} \sqrt{u}}{4 c_{15} \tau}\right\}\right)=2 d e^{-u / 4}
$$

provided that $0 \leq u \leq y^{2} \cdot 2^{n}$. The relation (6.63) immediately follows from (6.64) and (6.65).

The relations $(6.61),(6.63)$ imply that

$$
\mathbb{E} e^{h \Delta_{3}} \mathbf{1}_{A} \leq 2^{N} \cdot(2 d+1)^{N} e^{N / 8}, \quad \text { where } h=\left(8 c_{42} d^{3 / 2} \tau\right)^{-1}
$$

and $1_{A}$ is the indicator function of the event $A$. By (6.55) and (6.56), we have $\Delta<x / 2+\Delta_{3}$, for $\omega \in A$. Therefore,

$$
\mathbb{P}\{\Delta \geq x\} \leq 1-\mathbb{P}\{A\}+e^{-h x / 2} \mathbb{E} e^{h \Delta_{3}} \mathbf{1}_{A} .
$$

Now (6.48) follows via $(6.57),(6.66)$ and $(6.67)$.

Let the quantities $\varepsilon, x_{0}>0$ be defined by the equalities $\varepsilon=\frac{1}{2 c_{39} d^{3 / 2} \tau}$, $e^{e x_{0}}=(15 d)^{N}$. Integrating by parts and using (6.48), we obtain

$$
\begin{gathered}
\mathbb{E} e^{\varepsilon \Delta}=1+\int_{0}^{\infty} \varepsilon e^{\varepsilon x} \mathbb{P}\{\Delta \geq x\} d x, \\
\int_{0}^{x_{0}} \varepsilon e^{\varepsilon x} \mathbb{P}\{\Delta \geq x\} d x \leq \int_{0}^{x_{0}} \varepsilon e^{\varepsilon x} d x=e^{\varepsilon x_{0}}-1=(15 d)^{N}-1, \\
\int_{x_{0}}^{\infty} \varepsilon e^{\varepsilon x} \mathbb{P}\{\Delta \geq x\} d x \leq \int_{x_{0}}^{\infty} \varepsilon e^{-\varepsilon\left(x-x_{0}\right)} d x=1,
\end{gathered}
$$

and, hence, $\mathbb{E} e^{\varepsilon \Delta} \leq(15 d)^{N}+1 \leq(16 d)^{N}$. Together with (6.16) this completes the proof of Theorem 6.4 . 


\section{Estimates IN THE DYADIC SCHEME \\ UNDER THE CONDITION OF COINCIDENCE OF MOMENTS OF THE FIRST THREE ORDERS}

Suppose that $p, N$ are natural numbers, $p \leq N, \tau \geq 1$, and $\widetilde{\xi}_{1}, \ldots, \widetilde{\xi}_{2^{N}}$ are random vectors with known distributions $\mathcal{L}\left(\widetilde{\xi}_{k}\right) \in \mathcal{A}_{d}(\tau), \mathbb{E} \widetilde{\xi}_{k}=0$, cov $\widetilde{\xi}_{k}=$ $I, k=1, \ldots, 2^{N}$. Let $\widetilde{\eta}_{k}$ be random vectors which have the same moments of the first three orders as the vectors $\widetilde{\xi}_{k}, \mathcal{L}\left(\widetilde{\eta}_{k}\right) \in \mathcal{A}_{d}(\tau), k=1, \ldots, 2^{N}$. Let $Y_{1}, \ldots, Y_{2^{N}}$ be independent random vectors with distributions $\mathcal{L}\left(Y_{k}\right)=$ $\Psi\left(\mathcal{L}\left(\widetilde{\eta}_{k}\right)\right), k=1, \ldots, 2^{N}$, where the distributions $\Psi(\cdot)$ are defined in Section 6 . Consider as well the random vectors $\xi_{1}, \ldots, \xi_{2^{N}}$ with $\mathcal{L}\left(\xi_{l}\right)=\mathcal{L}\left(Y_{l}\right)$ for $l=(m-1) \cdot 2^{p}+1, m=1, \ldots, 2^{N-p}$, and with $\mathcal{L}\left(\xi_{l}\right)=\mathcal{L}\left(\widetilde{\xi}_{l}\right)$ otherwise. Let the random vectors $X_{k}, \mathcal{L}\left(X_{k}\right)=\mathcal{L}\left(\xi_{k}\right) ; V_{n, k}, \tilde{V}_{n, k}$, and $U_{n, k}, \widetilde{U}_{n, k}$ be constructed as functions of $Y_{1}, \ldots, Y_{2}$ as in Section 5.

Remark 7.1. From Lemma 6.1 and from the definition of the distributions $\Psi(\cdot)$ it follows that the vectors $\xi_{k}, X_{k}, Y_{k}$ have the same moments of the first three orders for each $k=1, \ldots, 2^{N}$.

Let $c_{1}, \ldots, c_{42}$ be absolute positive constants from Section 6 . By virtue of (6.14) we have

$$
\mathcal{L}\left(X_{k}\right), \mathcal{L}\left(Y_{k}\right) \in \mathcal{A}_{d}\left(c_{15} \tau\right), \quad k=1, \ldots, 2^{N} .
$$

LEMMA 7.2. There exist absolute positive constants $c_{43}, \ldots, c_{48}$ such that

a) if $1 \leq n \leq N, 0 \leq k<2^{N-n}, \tau^{2} d^{3} p / 2^{n-p} \leq c_{43}$, then

$$
\begin{array}{r}
\left|\widetilde{U}_{n, k}-\widetilde{V}_{n, k}\right| \leq \frac{c_{44} d^{2} \tau^{2}}{2^{n / 2}}\left(1+2^{-3 n / 2} \max \left\{\left|U_{n, k}\right|^{3},\left|\widetilde{U}_{n, k}\right|^{3}\right\}\right) \\
+\frac{1}{8}\left|U_{n, k}-V_{n, k}\right|
\end{array}
$$

provided that $\max \left\{\left|U_{n, k}\right|,\left|V_{n, k}\right|,\left|\widetilde{U}_{n, k}\right|\right\} \leq \frac{c_{45} \cdot 2^{n}}{d^{3 / 2} \tau}$

b) if $\tau^{2} d^{3} p / 2^{N-p} \leq c_{46}$, then

$$
\left|U_{N, 0}-V_{N, 0}\right| \leq c_{47} d^{2} \tau^{2} \cdot 2^{-N / 2}\left(1+2^{-3 N / 2}\left|U_{N, 0}\right|^{3}\right)
$$

provided that $\left|U_{N, 0}\right| \leq \frac{c_{48} \cdot 2^{N}}{d^{3 / 2} \tau}$.

Proof. At first we prove assertion a). Suppose that the vectors $L_{n, k}^{*}, L_{n, k}$ are constructed from coordinates of vectors $U_{n-1,2 k}, U_{n-1,2 k+1} ; U_{n, k}, \widetilde{U}_{n, k}$ as in the proof of Lemma 6.5. Let the vectors $R_{n, k}^{*}, R_{n, k}$ be analogously constructed from coordinates of vectors $V_{n-1,2 k}, V_{n-1,2 k+1} ; V_{n, k}, \widetilde{V}_{n, k}$. It is evident that the vectors $U_{n-1,2 k}$ and $U_{n-1,2 k+1}$ are as before independent. The same may be said about the vectors $V_{n-1,2 k}, V_{n-1,2 k+1}$. Using the relations $(5.2),(7.1)$, the properties of classes $\mathcal{A}_{d}(\tau)$, the definition of classes $\mathcal{R}_{d}(\tau, \varepsilon)$, Lemma 2.8 and Remarks $2.3,7.1$, we can easily verify that $\left(\mathcal{L}\left(L_{n, k}^{*}\right), \mathcal{L}\left(R_{n, k}^{*}\right)\right) \in \mathcal{R}_{2 d}\left(c_{15} \tau, 0\right)$. The relations (5.6), (5.12) show that $L_{n, k}=A L_{n, k}^{*}, R_{n, k}=A R_{n, k}^{*}$, where $A: \mathbb{R}^{2 d} \rightarrow \mathbb{R}^{2 d}$ is a linear operator with $\|A\|=\sqrt{2}$. Therefore, using Corollary 2.6, we see that

$$
\left(\mathcal{L}\left(L_{n, k}\right), \mathcal{L}\left(R_{n, k}\right)\right) \in \mathcal{R}_{2 d}\left(c_{15} \sqrt{2} \tau, 0\right)
$$


Now we define the vectors $L_{n, k}^{j}, R_{n, k}^{j}, j=d, \ldots, 2 d$, consisting of the first $j$ coordinates of the vectors $L_{n, k}, R_{n, k}$ respectively (see $(6.42)$ ). Below we show that the distributions $\mathcal{L}\left(L_{n, k}^{j}\right), \mathcal{L}\left(R_{n, k}^{j}\right), j=d+1, \ldots, 2 d$, satisfy in $j$-dimensional situation the conditions of Lemmas 2.15, 4.1 with $D=2^{n} I, \sigma^{2}=2^{n}$ and with the change of $d$ by $j$ and $\tau$ by $c_{34} \tau$, where $c_{34}=\max \left\{c_{15} \sqrt{2}, c_{1}^{-1}, c_{6}^{-1}, 1\right\}$ was already defined above. For this one should fix $j$ and verify that, if $\tau^{2} d^{3} p / 2^{n-p} \leq c_{49}$ with sufficiently small $c_{49}$, then for $h^{*} \in \mathbb{R}^{j},\left\|h^{*}\right\| c_{34} \tau<1$, and for all $v^{*} \in \mathbb{R}^{j}$ the inequalities (6.43), (6.44) hold, where $F_{h^{*}}=\bar{F}\left(h^{*}\right), F=\mathcal{L}\left(L_{n, k}^{j}\right)$ or $F=\mathcal{L}\left(R_{n, k}^{j}\right)$, $h^{*}=\left(h_{1}^{*}, \ldots, h_{d}^{*}\right)$ and the relations $(6.45)$ are satisfied (see $(2.20)$ and Definition 2.12). Besides, we need the validity of analogous relations in the $(j-1)$-dimensional situation for the distributions $F^{\prime}$. Finally, one should take into account the relation (7.4). It is clear that it suffices to check the conditions $(6.43),(6.44)$ for $F=\mathcal{L}\left(L_{n, k}^{j}\right), j=d, \ldots, 2 d$, if $c_{49}$ is small enough. For $F=\mathcal{L}\left(R_{n, k}^{j}\right)$ these conditions can be verified analogously.

Choosing $c_{49}$ to be sufficiently small and taking into account that $\tau \geq 1$, we ensure the validity of the inequality

$$
2^{n-p-2} \geq 2
$$

From the definition of distributions $\Psi(\cdot)$ and random vectors $L_{n, k}^{j}$ and from $(2.2),(5.1),(5.2),(5.12),(7.5)$ it is easy to deduce that (in the conditions of Section 7 )

$$
\left|\widehat{F}_{h^{*}}\left(t^{*}\right)\right| \leq \prod_{m=1}^{j-d}\left|\widehat{H}_{h_{m}^{*}}^{*}\left(t_{m}^{*}\right)\right|^{2^{n-p-1}} \prod_{m=j-d+1}^{d}\left|\widehat{H}_{h_{m}}\left(t_{m}\right)\right|^{2^{n-p}}
$$

By definition of $c_{34}$, we can use for estimating $\left|\widehat{H}_{h_{m}}\left(t_{m}\right)\right|$ the inequalities $(6.2)-(6.4)$ and for estimating $\left|\widehat{H}_{h_{m}^{*}}^{*}\left(t_{m}^{*}\right)\right|$ the inequalities $(6.5)-(6.7)$. In particular, from $(6.2),(6.3),(6.5),(6.6),(6.45)$ one can easily derive that

$$
\begin{aligned}
\sup _{t \in T} \prod_{m=1}^{j-d}\left|\widehat{H}_{h_{m}^{*}}^{*}\left(t_{m}^{*}\right)\right| \prod_{m=j-d+1}^{d}\left|\widehat{H}_{h_{m}}\left(t_{m}\right)\right|^{2} & \leq \max \left\{\exp \left(-\left(48 c_{34}^{2} \tau^{2} d^{2}\right)^{-1}\right), c_{3}^{2}, c_{8}\right\} \\
& \leq \exp \left(-\frac{c_{50}}{\tau^{2} d^{2}}\right)
\end{aligned}
$$

From (7.6), (7.7) it follows that, for $t^{*} \in T$,

$$
\left|\widehat{F}_{h^{*}}\left(t^{*}\right)\right| \leq \exp \left(-\frac{2^{n-p-2} c_{50}}{\tau^{2} d^{2}}\right) \prod_{m=1}^{j-d}\left|\widehat{H}_{h_{m}^{*}}^{*}\left(t_{m}^{*}\right)\right|^{2^{n-p-2}} \prod_{m=j-d+1}^{d}\left|\widehat{H}_{h_{m}}\left(t_{m}\right)\right|^{2^{n-p-1}} .
$$

Using (6.3), (6.6), we obtain

$$
\begin{gathered}
\int_{\left|t_{m}^{*}\right| \leq c_{7}}\left|\widehat{H}_{h_{m}^{*}}^{*}\left(t_{m}^{*}\right)\right|^{2^{n-p-2}} d t_{m}^{*} \leq \int_{|t| \leq c_{7}, t \in \mathbb{R}^{2}} \exp \left(-\frac{2^{n-p} \|\left. t\right|^{2}}{12}\right) d t \leq \frac{c}{2^{n-p}}, \\
\int_{\left|t_{m}\right| \leq c_{2}}\left|\widehat{H}_{h_{m}}\left(t_{m}\right)\right|^{2^{n-p-1}} d t_{m} \leq \int_{|t| \leq c_{2}, t \in \mathbb{R}^{1}} \exp \left(-\frac{2^{n-p} t^{2}}{12}\right) d t \leq \frac{c}{2^{(n-p) / 2}} .
\end{gathered}
$$


Analogously, using (6.2), (6.4), (6.5), (6.7), (7.5), we have

$$
\begin{aligned}
\int_{\left|t_{m}^{*}\right|>c_{7}}\left|\widehat{H}_{h_{m}^{*}}^{*}\left(t_{m}^{*}\right)\right|^{2^{n-p-2}} d t_{m}^{*} & \leq \int_{|t|>c_{7}} c_{8}^{2^{n-p-3}}\left|\widehat{H}_{h_{m}^{*}}^{*}(t)\right| d t \\
& \leq c \exp \left(-c \cdot 2^{n-p}\right) \leq \frac{c}{2^{n-p}}, \\
\int_{\left|t_{m}\right|>c_{2}}\left|\widehat{H}_{h_{m}}\left(t_{m}\right)\right|^{2^{n-p-1}} d t_{m} & \leq \int_{|t|>c_{2}} c_{3}^{2^{n-p-2}}\left|\widehat{H}_{h_{m}}(t)\right| d t \\
& \leq c \exp \left(-c \cdot 2^{n-p}\right) \leq \frac{c}{2^{(n-p) / 2}} .
\end{aligned}
$$

From (7.9), (7.10) it follows that

$$
\int_{\mathbb{R}^{2}}\left|\widehat{H}_{h_{m}^{*}}^{*}\left(t_{m}^{*}\right)\right|^{2^{n-p-2}} d t_{m}^{*} \leq \frac{c}{2^{n-p}}, \quad \int_{\mathbb{R}^{1}}\left|\widehat{H}_{h_{m}}\left(t_{m}\right)\right|^{2^{n-p-1}} d t_{m} \leq \frac{c}{2^{(n-p) / 2}} .
$$

The inequalities (7.8), (7.11) imply that

$$
(2 \pi)^{-j} \int_{T}\left|\widehat{F}_{h^{*}}\left(t^{*}\right)\right| d t^{*} \leq \frac{1}{2^{p} \cdot 2^{n j / 2}(2 \pi)^{j / 2}} \exp \left(c_{51} d p-\frac{c_{52} \cdot 2^{n-p}}{\tau^{2} d^{2}}\right) .
$$

Choosing $c_{49}$ to be small enough, we can ensure the validity of the inequalities

$$
\exp \left(c_{51} d p-\frac{c_{52} \cdot 2^{n-p}}{\tau^{2} d^{2}}\right) \leq \frac{c_{34}^{2} \tau^{2} d^{2}}{2^{n-p}} \leq \frac{c_{34}^{2} \tau^{2} j^{2}}{2^{n-p}} \leq \frac{4 c_{34}^{2} \tau^{2} d^{2}}{2^{n-p}} \leq \frac{1}{4}
$$

The inequalities (7.12), (7.13) imply (6.43).

For the proof of (6.44) we apply the Hölder inequality and obtain that

$$
\int_{T}\left|\left\langle t^{*}, v^{*}\right\rangle \widehat{F}_{h^{*}}\left(t^{*}\right)\right| d t^{*} \leq\left\|v^{*}\right\|\left(\int_{T}\left|\widehat{F}_{h^{*}}\left(t^{*}\right)\right| d t^{*} \int_{T}\left\|t^{*}\right\|^{2}\left|\widehat{F}_{h^{*}}\left(t^{*}\right)\right| d t^{*}\right)^{1 / 2} \text {. }
$$

For estimating the first integral in the right-hand side of (7.14) we note that, according to $(7.12),(7.13)$,

$$
(2 \pi)^{-j} \int_{T}\left|\widehat{F}_{h^{*}}\left(t^{*}\right)\right| d t^{*} \leq \frac{1}{4(2 \pi)^{j / 2} \cdot 2^{n j / 2}} .
$$

Let us estimate the second integral. By $(6.3),(6.6)$, we have

$$
\begin{aligned}
\int_{\left|t_{m}^{*}\right| \leq c_{7}}\left\|t_{m}^{*}\right\|^{2}\left|\hat{H}_{h_{m}^{*}}^{*}\left(t_{m}^{*}\right)\right|^{2^{n-p-2}} d t_{m}^{*} & \leq \int_{|t| \leq c_{7}, t \in \mathbb{R}^{2}}\|t\|^{2} \exp \left(-\frac{2^{n-p}\|t\|^{2}}{12}\right) d t \\
& \leq \frac{c}{2^{2(n-p)}}, \\
\int_{\left|t_{m}\right| \leq c_{2}} t_{m}^{2}\left|\widehat{H}_{h_{m}}\left(t_{m}\right)\right|^{2^{n-p-1}} d t_{m} & \leq \int_{|t| \leq c_{2}, t \in \mathbb{R}^{1}} t^{2} \exp \left(-\frac{2^{n-p} t^{2}}{12}\right) d t \\
& \leq \frac{c}{2^{3(n-p) / 2}} .
\end{aligned}
$$


Moreover, using $(6.2),(6.4),(6.5),(6.7),(7.5)$, we obtain

$$
\begin{aligned}
\int_{\left|t_{m}^{*}\right|>c_{7}}\left\|t_{m}^{*}\right\|^{2}\left|\widehat{H}_{h_{m}^{*}}^{*}\left(t_{m}^{*}\right)\right|^{2^{n-p-2}} d t_{m}^{*} & \leq \int_{|t|>c_{7}} c_{8}^{2^{n-p-3}} \|\left. t\right|^{2}\left|\widehat{H}_{h_{m}^{*}}^{*}(t)\right| d t \\
& \leq c \exp \left(-c \cdot 2^{n-p}\right) \leq \frac{c}{2^{2(n-p)}} \\
\int_{\left|t_{m}\right|>c_{2}} t_{m}^{2}\left|\widehat{H}_{h_{m}}\left(t_{m}\right)\right|^{2^{n-p-1}} d t_{m} & \leq \int_{|t|>c_{2}} c_{3}^{2^{n-p-2}} t^{2}\left|\widehat{H}_{h_{m}}(t)\right| d t \\
& \leq c \exp \left(-c \cdot 2^{n-p}\right) \leq \frac{c}{2^{3(n-p) / 2}} .
\end{aligned}
$$

From (7.16), (7.17) it follows that

$$
\begin{aligned}
\int_{\mathbb{R}^{2}}\left\|t_{m}^{*}\right\|^{2}\left|\hat{H}_{h_{m}^{*}}^{*}\left(t_{m}^{*}\right)\right|^{2^{n-p-2}} d t_{m}^{*} & \leq \frac{c}{2^{2(n-p)}}, \\
\int_{\mathbb{R}^{1}} t_{m}^{2}\left|\hat{H}_{h_{m}}\left(t_{m}\right)\right|^{2^{n-p-1}} d t_{m} & \leq \frac{c}{2^{3(n-p) / 2}} .
\end{aligned}
$$

Using now (7.8), (7.11), (7.18), we see that

$$
(2 \pi)^{-j} \int_{T}\left\|t^{*}\right\|^{2}\left|\widehat{F}_{h^{*}}\left(t^{*}\right)\right| d t^{*} \leq \frac{1}{2^{n}(2 \pi)^{j / 2} \cdot 2^{n j / 2}} \exp \left(c_{53} d p-\frac{c_{54} \cdot 2^{n-p}}{\tau^{2} d^{2}}\right) .
$$

Choosing $c_{49}$ to be small enough, we can ensure the validity of the inequality

$$
\exp \left(c_{53} d p-\frac{c_{54} \cdot 2^{n-p}}{\tau^{2} d^{2}}\right) \leq \frac{1}{4}
$$

The inequalities (7.14), (7.15), (7.19), (7.20) imply (6.44).

Using $(2.21),(4.2),(5.8),(5.9)$ and applying Lemma 4.1 and the inequality (2.22) of Lemma 2.15 (for the parameter $\widetilde{\tau}=c_{34} \tau$ ) in the $j$-dimensional case, $j=d+1, \ldots, 2 d$, we obtain that

$$
\begin{aligned}
\left|L_{n, k}^{(j)}-R_{n, k}^{(j)}\right| \leq \frac{c \tau^{2}}{2^{n / 2}}\left(j^{2}+\left(\Gamma_{j-1}^{2}\right.\right. & \left.+2^{n / 2} \tau^{-1} \Lambda_{j-1}\right)\left(j+\frac{\left|L_{n, k}^{(j)}\right|}{2^{n / 2}}\right)+\frac{\left|L_{n, k}^{(j)}\right|^{3}}{2^{3 n / 2}} \\
& \left.+\Gamma_{j-1}^{3}+2^{n / 2} \tau^{-1} \Lambda_{j-1} \Gamma_{j-1}\right)
\end{aligned}
$$

where

$$
\Lambda_{j-1}=2^{-n / 2} j^{1 / 2}\left|L_{n, k}^{j-1}-R_{n, k}^{j-1}\right|, \Gamma_{j-1}=2^{-n / 2} j^{1 / 2} \max \left\{\left|L_{n, k}^{j-1}\right|,\left|R_{n, k}^{j-1}\right|\right\},
$$

provided that

$$
\frac{\tau^{2} j^{3} p}{2^{n-p}} \leq c_{55},\left|L_{n, k}^{(j)}\right| \leq \frac{c_{56} \cdot 2^{n}}{j \tau}, \frac{\max \left\{\left|L_{n, k}^{j-1}\right|,\left|R_{n, k}^{j-1}\right|\right\}}{2^{n / 2}} \leq \frac{c_{57} \cdot 2^{n / 2}}{j^{3 / 2} \tau}
$$


and $c_{55}, c_{56}, c_{57}$ are small enough. Verifying the inequality (7.21), one should take into account that in Lemmas 2.15 and 4.1 the densities $v_{m}(\cdot), m=1,2$, are the densities of shifted conditional distributions. The possibility to write the inequality (7.21) and the conditions (7.23) without shift is ensured by choosing $c_{56}, c_{57}$ to be sufficiently small and by using the inequality (2.16) for $y_{m}, m=1,2$, involved in Lemma 2.15 .

Using again the possibility to choose $c_{55}, c_{56}, c_{57}$ to be small enough, we can easily derive from (7.21)-(7.23) that if the conditions (7.23) are satisfied, then

$$
\left|L_{n, k}^{(j)}-R_{n, k}^{(j)}\right| \leq \frac{c_{58} \tau^{2} d^{2}}{2^{n / 2}}\left(1+\frac{\left|L_{n, k}^{j}\right|^{3}}{2^{3 n / 2}}\right)+\frac{1}{16}\left|L_{n, k}^{j-1}-R_{n, k}^{j-1}\right| .
$$

For the proof of (7.24) one should estimate each summand in the righthand side of the inequality (7.21) separately. We use, in particular, that $\Gamma_{j-1} \leq \Lambda_{j-1}+2^{-n / 2} j^{1 / 2}\left|L_{n, k}^{j-1}\right|$.

Let us prove that, for $j=d+1, \ldots, 2 d$,

$$
\begin{gathered}
\left|L_{n, k}^{(j)}-R_{n, k}^{(j)}\right| \leq \frac{c_{44} \tau^{2} d^{2}}{2 \cdot 2^{n / 2}}\left(1+\frac{\left|L_{n, k}^{j}\right|^{3}}{2^{3 n / 2}}\right)+\frac{1}{16}\left|L_{n, k}^{j-1}-R_{n, k}^{j-1}\right| \\
\left|R_{n, k}^{(j)}\right| \leq \frac{c_{57} \cdot 2^{n}}{4 d^{3 / 2} \tau}
\end{gathered}
$$

provided that

$$
\frac{\tau^{2} d^{3} p}{2^{n-p}} \leq c_{43}, \quad \max \left\{\left|U_{n, k}\right|,\left|V_{n, k}\right|,\left|\tilde{U}_{n, k}\right|\right\} \leq \frac{c_{45} \cdot 2^{n}}{d^{3 / 2} \tau},
$$

where

$$
c_{44}=2 c_{58}, c_{45}=\min \left\{\frac{c_{56}}{2}, \frac{c_{57}}{16}, \frac{1}{\sqrt{c_{44}}}\right\}, c_{43}=\min \left\{\frac{c_{55}}{8},\left(\frac{c_{45}}{c_{44}}\right)^{2 / 3}\right\} .
$$

It is clear that (7.28) implies

$$
c_{59} \stackrel{\text { def }}{=} c_{45}+\frac{c_{44}}{2}\left(c_{43}^{3 / 2}+c_{45}^{3}\right)+\frac{c_{45}}{8}+\frac{c_{57}}{64} \leq \frac{c_{57}}{4} .
$$

We shall use the induction with respect to $j$. Inequality (7.25) in the case $j=d+1$ follows directly from (7.24), (7.28) and from the definition of vectors $L_{n, k}, R_{n, k}$, since the conditions (7.23) are in this case the consequences of (7.27), (7.28). Moreover, using (7.25), (7.27)-(7.29), we see that

$$
\left|R_{n, k}^{(d+1)}\right| \leq\left|L_{n, k}^{(d+1)}\right|+\left|L_{n, k}^{(d+1)}-R_{n, k}^{(d+1)}\right| \leq \frac{c_{59} \cdot 2^{n}}{d^{3 / 2} \tau} \leq \frac{c_{57} \cdot 2^{n}}{4 d^{3 / 2} \tau} .
$$

This completes the proof of (7.25), (7.26) for $j=d+1$.

Suppose now that the inequalities $(7.25)$ and $(7.26)$ are already proved for $j=d+1, \ldots, s-1, d+2 \leq s \leq 2 d$. We shall prove that these inequalities are valid for $j=s$. Inequality (7.25) for $j=s$ follows from (7.24), (7.28) 
since the validity of (7.23) can be easily derived in this case from (7.26) (with $j=d+1, \ldots, s-1),(7.27)$, (7.28). Moreover, using (7.25) (with $j=s$ ), (7.26) (with $j=d+1, \ldots, s-1),(7.27)-(7.29)$, we obtain the relation (7.30) with the change of the superscript $(d+1)$ by $(s)$. This completes the proof of $(7.25),(7.26)$ for $j=d+1, \ldots, 2 d$.

Inequality (7.2) follows from (7.25), (6.42) and from the definition of $L_{n, k}$ and $R_{n, k}$.

Let us prove assertion b). Consider the vectors $U_{N, 0}^{j}, V_{N, 0}^{j}, j=1, \ldots, d$, consisting of the first $j$ coordinates of the vectors $U_{N, 0}, V_{N, 0}$ respectively (see $(6.38)$ ). From the properties of classes $\mathcal{R}_{d}(\tau, \varepsilon)$, Corollary 2.6, Remarks $2.3,7.1$ and the relations $(5.2),(7.1)$ it follows that

$$
\left(\mathcal{L}\left(U_{N, 0}^{j}\right), \mathcal{L}\left(V_{N, 0}^{j}\right)\right) \in \mathcal{R}_{j}\left(c_{15} \tau, 0\right)
$$

One can verify that, if $\tau^{2} j^{3} p / 2^{N-p} \leq c_{60}$ with sufficiently small $c_{60}$, then the pairs of distributions $\left(\mathcal{L}\left(U_{N, 0}^{j}\right), \mathcal{L}\left(V_{N, 0}^{j}\right)\right)$ satisfy in $j$-dimensional situation the conditions of Lemmas 2.15 and 4.1 with $D=2^{N} I, \sigma^{2}=2^{N}$ and with the change of $\tau$ by $c_{24} \tau$, where $c_{24}=\max \left\{c_{15}, c_{1}^{-1}, 1\right\}$ was defined in Section 6 . For this one should verify that, for $h \in \mathbb{R}^{j},\|h\| c_{24} \tau<1$ and for all $v \in$ $\mathbb{R}^{j}$, the following inequalities hold, if $c_{60}$ is small enough (see (2.20) and Definition 2.12):

$$
\begin{gathered}
(2 \pi)^{-j} \int_{T}\left|\widehat{F}_{h}(t)\right| d t \leq \frac{c_{24}^{2} \tau^{2} j^{2}}{2^{N}(2 \pi)^{j / 2} \cdot 2^{N j / 2}}, \\
(2 \pi)^{-j} \int_{T}\left|\langle t, v\rangle \widehat{F}_{h}(t)\right| d t \leq \frac{\|v\|}{4 \cdot 2^{N / 2}(2 \pi)^{j / 2} \cdot 2^{N j / 2}},
\end{gathered}
$$

where $F_{h}=\bar{F}(h), F=\mathcal{L}\left(U_{N, 0}^{j}\right)$ or $F=\mathcal{L}\left(V_{N, 0}^{j}\right)$,

$$
T=\left\{t \in \mathbb{R}^{j}: 4\|t\| c_{24} \tau j \geq 1\right\} .
$$

The inequalities (7.32), (7.33) automatically imply the validity of analogous relations in the $(j-1)$-dimensional situation, $j=2, \ldots, d$, for the distributions $F^{\prime}$. It is clear that it suffices to check the conditions (7.32), (7.33) for $F=\mathcal{L}\left(U_{N, 0}^{j}\right), j=1, \ldots, d$, if $c_{60}$ is small enough. For $F=\mathcal{L}\left(V_{N, 0}^{j}\right)$ these conditions can be verified analogously.

From the definition of distributions $\Psi(\cdot)$ and random vectors $U_{N, 0}^{j}$ and from $(2.2),(5.1),(5.2)$ one can easily derive that

$$
\left|\widehat{F}_{h}(t)\right| \leq \prod_{m=1}^{j}\left|\widehat{H}_{h_{m}}\left(t_{m}\right)\right|^{2^{N-p}} .
$$

By the definition of $c_{24}$, we can use for estimating $\left|\widehat{H}_{h_{m}}\left(t_{m}\right)\right|$ the inequalities $(6.2)-(6.4)$. The rest of the proof of inequalities $(7.32),(7.33)$ does not practically differ from the proof of $(6.19),(6.20)$ and is simpler than the proof of $(6.43),(6.44)$ in Section 7 . Therefore it is omitted. The only difference is in the change of constants and notation. 
Using the relations $(2.21),(4.2),(5.7)$ and applying Lemma 4.1 and the inequality (2.22) of Lemma 2.15 in the $j$-dimensional case, $j=1, \ldots, d$, we obtain that

$$
\left|U_{N, 0}^{(1)}-V_{N, 0}^{(1)}\right| \leq \frac{c_{61} \tau^{2}}{2^{N / 2}}\left(1+\frac{\left|U_{N, 0}^{(1)}\right|^{3}}{2^{3 N / 2}}\right),
$$

if

$$
\frac{\tau^{2} p}{2^{N-p}} \leq c_{62}, \quad\left|U_{N, 0}^{(1)}\right| \leq \frac{c_{63} \cdot 2^{N}}{\tau},
$$

and, for $2 \leq j \leq d$,

$$
\left|U_{N, 0}^{(j)}-V_{N, 0}^{(j)}\right| \leq \frac{c_{61} \tau^{2} d^{2}}{2^{N / 2}}\left(1+\frac{\left|U_{N, 0}^{j}\right|^{3}}{2^{3 N / 2}}\right)+\frac{1}{2}\left|U_{N, 0}^{j-1}-V_{N, 0}^{j-1}\right|,
$$

if

$$
\frac{\tau^{2} j^{3} p}{2^{N-p}} \leq c_{62},\left|U_{N, 0}^{(j)}\right| \leq \frac{c_{63} \cdot 2^{N}}{j \tau}, \frac{\max \left\{\left|U_{N, 0}^{j-1}\right|,\left|V_{N, 0}^{j-1}\right|\right\}}{2^{N / 2}} \leq \frac{c_{64} \cdot 2^{N / 2}}{j^{3 / 2} \tau}
$$

and $c_{62}, c_{63}, c_{64}$ are small enough. Checking inequalities (7.36), (7.38) should be carried out analogously to the proof of (7.24).

Let us prove that, for $j=1, \ldots, d$,

$$
\begin{gathered}
\left|V_{N, 0}^{(j-1)}\right| \leq \frac{c_{64} \cdot 2^{N}}{d^{3 / 2} \tau} \\
\left|U_{N, 0}^{(j)}-V_{N, 0}^{(j)}\right| \leq \frac{c_{47} \tau^{2} d^{2}}{2 \cdot 2^{N / 2}}\left(1+\frac{\left|U_{N, 0}^{j}\right|^{3}}{2^{3 N / 2}}\right)+\frac{1}{2}\left|U_{N, 0}^{j-1}-V_{N, 0}^{j-1}\right|,
\end{gathered}
$$

if

$$
\frac{\tau^{2} d^{3} p}{2^{N-p}} \leq c_{46}, \quad\left|U_{N, 0}\right| \leq \frac{c_{48} \cdot 2^{N}}{d^{3 / 2} \tau}
$$

where

$$
\begin{gathered}
c_{47}=2 c_{61}, \quad c_{48}=\min \left\{c_{63}, \frac{c_{64}}{8}, \frac{1}{\sqrt{c_{47}}}\right\}, \quad c_{46}=\min \left\{c_{62},\left(\frac{c_{48}}{c_{47}}\right)^{2 / 3}\right\}, \\
\left|V_{N, 0}^{(0)}\right|=\left|U_{N, 0}^{0}-V_{N, 0}^{0}\right|=0 .
\end{gathered}
$$

It is clear that (7.43) implies

$$
c_{65} \stackrel{\text { def }}{=} c_{48}+\frac{c_{47}}{2}\left(c_{46}^{3 / 2}+c_{48}^{3}\right)+\frac{c_{48}}{2}+\frac{c_{64}}{2} \leq c_{64} .
$$

We shall use the induction with respect to $j$. Inequality (7.41) for $j=1$ follows directly from $(7.36),(7.43)$, since the conditions $(7.37)$ are in this case the consequences of $(7.42),(7.43)$. Inequality $(7.40)$ for $j=1$ is valid automatically (see (7.43)). 
Suppose now that the inequalities (7.40) and (7.41) are already proved for $j=1, \ldots, s-1,2 \leq s \leq d$. We shall prove that these inequalities are valid for $j=s$. Using (7.40) (with $j=1, \ldots, s-1$ ), (7.41) (with $j=s-1),(7.42)-(7.44)$, we obtain

$$
\left|V_{N, 0}^{(s-1)}\right| \leq\left|U_{N, 0}^{(s-1)}\right|+\left|U_{N, 0}^{(s-1)}-V_{N, 0}^{(s-1)}\right| \leq \frac{c_{65} \cdot 2^{N}}{d^{3 / 2} \tau} \leq \frac{c_{64} \cdot 2^{N}}{d^{3 / 2} \tau}
$$

Moreover, it is clear that the inequality (7.41) for $j=s$ can be easily derived from (7.38), (7.43) since the validity of (7.39) follows in this case from (7.40) (with $j=1, \ldots, s-1),(7.42),(7.43),(7.45)$. This completes the proof of $(7.40),(7.41)$ for $j=1, \ldots, d$.

Inequality (7.3) follows now from (7.41)-(7.43) and from the definition of $U_{N, 0}, U_{N, 0}^{j}, U_{N, 0}^{(j)}, V_{N, 0}, V_{N, 0}^{j}, V_{N, 0}^{(j)}$.

\section{Proof of Theorem 1.5}

In this section it is convenient to assume that the constants $c_{1}, \ldots, c_{65}$ are the same as in Sections 6 and 7 . Let $u, v, w$ be arbitrary numbers satisfying the relations

$$
\frac{5}{8} \leq v<\frac{2}{3}, \quad u=1-v, \quad w=2-3 v .
$$

It is clear that

$$
\frac{1}{3}<u \leq \frac{3}{8}, \quad 0<w \leq \frac{1}{8}, \quad 3 u-w=1 .
$$

Below the symbols $a_{1}, \ldots, a_{7}$ will be used for denoting positive numbers depending on $v$ only. The letter $a$ can denote different positive quantities depending only on $v$ when we do not need to fix their numerical values. It is clear that, if $v$ is fixed, $a, a_{1}, \ldots, a_{7}$ are absolute constants. Denote

$$
\begin{gathered}
c_{66}=\max \left\{c_{44}, c_{47}\right\}, \quad c_{67}=\min \left\{c_{45}, c_{48}, 1\right\}, \quad c_{68}=\min \left\{c_{43}, c_{46}\right\}, \\
a_{1}=\min \left\{\frac{c_{67}}{5}, \frac{1}{\left(126 c_{66}\right)^{1 / 2}}, \frac{1-2^{-w}}{2}\right\}, \quad a_{2}=\min \left\{c_{68}, a_{1}^{2}, 1\right\} .
\end{gathered}
$$

Let $\tau \geq 1, \lambda \geq 1$. Define $p$ as the minimal integer satisfying the inequalities

$$
p \geq 4, \quad \frac{a_{1} \cdot 2^{v p}}{d^{3 / 2} \tau} \geq 4 \lambda .
$$

Denote by $M$ the maximal integer such that

$$
\frac{\tau^{2} d^{3} p}{2^{M-p}} \geq a_{2}
$$

Let $N$ be a positive integer such that

$$
\frac{\tau^{2} d^{3} p}{2^{N-p}}<a_{2}, \quad \frac{N-M}{p} \text { is integer. }
$$


Obviously, the relations (8.3)-(8.6) imply

$$
N>M \geq p, \quad N \geq 2 p .
$$

Let $\widetilde{\xi}_{1}, \ldots, \widetilde{\xi}_{2^{N}}$ be random vectors with distributions $\mathcal{L}\left(\widetilde{\xi}_{k}\right) \in \mathcal{A}_{d}(\tau), \mathbb{E} \widetilde{\xi}_{k}$ $=0, \operatorname{cov} \widetilde{\xi}_{k}=I, k=1, \ldots, 2^{N}$. Let $\widetilde{\eta}_{k}$ be random vectors which have the same moments of the first three orders as the vectors $\widetilde{\xi}_{k}, \mathcal{L}\left(\widetilde{\eta}_{k}\right) \in \mathcal{A}_{d}(\tau)$ and $\mathbb{P}\left\{\left|\widetilde{\eta}_{k}\right| \leq \lambda\right\}=1, k=1, \ldots, 2^{N}$. Suppose that the independent random vectors $\tilde{Y}_{k}, k=1, \ldots, 2^{N}$, with distributions $\mathcal{L}\left(\tilde{Y}_{k}\right)=\Psi\left(\mathcal{L}\left(\widetilde{\eta}_{k}\right)\right)$ are already constructed (the distributions $\Psi(\cdot)$ are defined in Section 6 ). According to Lemma $6.1, \widetilde{Y}_{k}$ and $\widetilde{\xi}_{k}$ have the same moments of the first three orders. Our aim is to construct independent random vectors $\widetilde{X}_{k}, k=1, \ldots, 2^{N}$, with $\mathcal{L}\left(\widetilde{X}_{k}\right)=\mathcal{L}\left(\widetilde{\xi}_{k}\right)$ so that the sequential sums of $\widetilde{X}_{k}$ and $\widetilde{Y}_{k}$ would be close with large probability. For this we define the random vectors

$$
\left\{Y_{k}^{[N-l p]}, Z_{(k-1) \cdot 2^{l p}+1}^{[N-l p]}, 1 \leq k \leq 2^{N-l p}, 0 \leq l \leq(N-M-p) / p\right\}
$$

with the help of a recurrent procedure described below. Note that in onedimensional case a very similar procedure was used by Sakhanenko (1984).

At first, we set $Y_{k}^{[N]}=\tilde{Y}_{k}, k=1, \ldots, 2^{N}$. After this, using the procedure described in Sections 5 and 7 , we define the independent random vectors $Z_{k}^{[N]}, k=1, \ldots, 2^{N}$, with $\mathcal{L}\left(Z_{r}^{[N]}\right)=\Psi\left(\mathcal{L}\left(\widetilde{\eta}_{r}\right)\right)$ for $r=(m-1) \cdot 2^{p}+1$, $m=1, \ldots, 2^{N-p}$. For $r=1, \ldots, 2^{N}, r \neq(m-1) \cdot 2^{p}+1, m=1, \ldots, 2^{N-p}$, we take $\mathcal{L}\left(Z_{r}^{[N]}\right)=\mathcal{L}\left(\widetilde{\xi}_{r}\right)$ and define $\widetilde{X}_{r}=Z_{r}^{[N]}$. Thus, the amount of vectors $\widetilde{X}_{k}$, which are not constructed, is decreased in $2^{p}$ times. In the case $N=M+p$, the first step of applying this procedure is simultaneously the last one, since the random vectors (8.8) with $l=(N-M-p) / p=0$ are already constructed.

Suppose now that $N>M+p$. Then we repeat the same procedure, working only with vectors which have the distributions $\Psi\left(\mathcal{L}\left(\widetilde{\eta}_{r}\right)\right)$ and diminishing their number after each step in $2^{p}$ times. Namely, if the vectors $\left\{Y_{k}^{[N-l p+p]}, Z_{(k-1) \cdot 2^{l p-p}+1}^{[N-l p+p]}, 1 \leq k \leq 2^{N-l p+p}\right\}$ are already defined for an integer $l$ and $1 \leq l \leq(N-M-p) / p$, we set $Y_{k}^{[N-l p]}=Z_{(k-1) \cdot 2^{l p}+1}^{[N-l p+p]}, k=$ $1, \ldots, 2^{N-l p}$, and then, using again the dyadic scheme described in Sections 5 and 7 , construct the independent random vectors $Z_{(k-1) \cdot 2^{l_{p}}+1}^{\left[N-l_{p}\right]}, k=$ $1, \ldots, 2^{N-l p}$, distributed as follows. In the case $r=(m-1) \cdot 2^{l p+p}+1$, $m=1, \ldots, 2^{N-l p-p}$, we choose $\mathcal{L}\left(Z_{r}^{[N-l p]}\right)=\Psi\left(\mathcal{L}\left(\widetilde{\eta}_{r}\right)\right)$. In the opposite case, when $r=(s-1) \cdot 2^{l p}+1, s=1, \ldots, 2^{N-l p}$, and when $r$ cannot be represented in the form $r=(m-1) \cdot 2^{l p+p}+1$, for some $m=1, \ldots, 2^{N-l p-p}$, we take $\mathcal{L}\left(Z_{r}^{[N-l p]}\right)=\mathcal{L}\left(\widetilde{\xi}_{r}\right)$ and define $\widetilde{X}_{r}=Z_{r}^{[N-l p]}$.

After the last step (with $l=(N-M-p) / p)$ all independent random vectors $\tilde{X}_{i}, i=1, \ldots, 2^{N}$, are constructed except $\widetilde{X}_{r}$ having indices of the form $r=(m-1) \cdot 2^{N-M}+1, m=1, \ldots, 2^{M}$. Instead of this we have constructed the vectors with distributions $\mathcal{L}\left(Z_{r}^{[M+p]}\right)=\Psi\left(\mathcal{L}\left(\widetilde{\eta}_{r}\right)\right)$ for $r=(m-1) \cdot 2^{N-M}+1, m=1, \ldots, 2^{M}$. Together with $\tilde{X}_{i}$ these vectors form a collection of mutually independent vectors. This can be easily verified 
by induction, if we take into account that, in each step, the new collection of independent vectors is constructed as a function of the vectors which are independent of all already constructed vectors $\widetilde{X}_{i}$.

In order to add the missing elements to the sequence of already constructed $\tilde{X}_{i}$, we define the random vectors $Y_{k}^{*}=Y_{k}^{[M]}=Z_{(k-1) \cdot 2^{N-M}+1}^{[M+p]}$, $k=1, \ldots, 2^{M}$, and, using again the procedure described in Section 5, construct the independent random vectors $X_{k}^{*}, k=1, \ldots, 2^{M}$, with distributions $\mathcal{L}\left(X_{k}^{*}\right)=\mathcal{L}\left(\widetilde{\xi}_{(k-1) \cdot 2^{N-M}+1}\right)$. Then we set $\widetilde{X}_{(k-1) \cdot 2^{N-M}+1}=X_{k}^{*}$. It is clear that the vectors $X_{k}^{*}$ are mutually independent and they do not depend on $\widetilde{X}_{i}$ constructed earlier, since, by construction, $X_{k}^{*}$ are functions of $Y_{1}^{*}, \ldots, Y_{2^{M}}^{*}$. This completes the procedure of constructing $\tilde{X}_{i}$, $i=1, \ldots, 2^{N}$.

By analogy with Sakhanenko (1984), we shall prove that the procedure described above provides good approximation, using an induction with respect to $N$. It is clear that the procedure of constructing the independent random vectors $X_{*, k}=\widetilde{X}_{(k-1) \cdot 2^{p}+1}, k=1, \ldots, 2^{N-p}$, coincides with the procedure of constructing the vectors $\widetilde{X}_{k}, k=1, \ldots, 2^{N}$. The only difference is in the change of $N$ by $N-p$. Thus, below we shall assume that we have the good approximation in the case when we construct $2^{N-p}$ summands and prove the analogous statement in the case of $2^{N}$ summands.

We shall use the notation which was already used in Sections 5 and 7 with $Y_{k}=Y_{k}^{[N]}, X_{k}=Z_{k}^{[N]}, k=1, \ldots, 2^{N}$. Denote $S=S_{j}, T=T_{j}$ (see (5.5), (5.11)). Sometimes we shall omit for simplicity the index $j, 1 \leq j \leq 2^{N}$, keeping in mind that it is fixed. By virtue of (6.14), by construction and by the definition of distributions $\Psi(\cdot)$ we have

$$
\mathbb{P}\left\{\left|Y_{k}\right| \leq 4 \lambda\right\}=1, \quad k=1, \ldots, 2^{N} .
$$

It is clear that from (5.5), (8.9) it follows that

$$
\mathbb{P}\left\{|T| \leq 4 \lambda \cdot 2^{N}\right\}=1 .
$$

For fixed integers $j, 1 \leq j \leq 2^{N}$, and $s, 0 \leq s \leq N$, define the integer $k_{s}=$ $k_{s}(j)$ by the relation $\left(k_{s}-1\right) \cdot 2^{s}<j \leq k_{s} \cdot 2^{s}$. Now we can represent the sums $S$ and $T$ in the form

$$
S=S^{(s)}-S^{\{s\}}, \quad T=T^{(s)}-T^{\{s\}},
$$

where

$$
\begin{array}{ll}
S^{(s)}=S_{k_{s} \cdot 2^{s}}, & S^{\{s\}}=S^{(s)}-S, \\
T^{(s)}=T_{k_{s} \cdot 2^{s}}, & T^{\{s\}}=T^{(s)}-T .
\end{array}
$$

According to Corollary 5.3 and Lemma 5.1,

$$
\left|S^{(s)}-T^{(s)}\right| \leq\left|U_{N, 0}-V_{N, 0}\right|+\sum_{n=s+1}^{N}\left|\tilde{U}^{(n)}-\tilde{V}^{(n)}\right|,
$$

where $\tilde{U}^{(n)}=\tilde{U}_{n, l_{n, j}}, \quad \tilde{V}^{(n)}=\tilde{V}_{n, l_{n, j}}, l_{n, j} \cdot 2^{n}<j \leq\left(l_{n, j}+1\right) \cdot 2^{n}$ (see $(5.13)$ ). As before, $l_{n, j}$ is the integer such that $U_{n, l_{n, j}}$, the sum over 
a block of $2^{n}$ summands, contains the summand $X_{j}$ and, consequently, the summand $X_{k_{s} \cdot 2^{s}}$, for $s<n \leq N$. Denote as well $U^{(n)}=U_{n, l_{n, j}}$, $V^{(n)}=V_{n, l_{n, j}}$. It is evident that $U^{(N)}=U_{N, 0}, V^{(N)}=V_{N, 0}$. Therefore, from $(8.11),(8.13)$ it follows that

$$
|S-T| \leq\left|U^{(N)}-V^{(N)}\right|+\sum_{n=s+1}^{N}\left|\tilde{U}^{(n)}-\tilde{V}^{(n)}\right|+\left|S^{\{s\}}\right|+\left|T^{\{s\}}\right| .
$$

Define the random integer $\mu=\mu_{j}$ by $\mu=N+1$, if at least one of the following inequalities is valid:

$$
\frac{\tau^{2} d^{3} p}{2^{N-p}}>a_{2}, \quad\left|S^{\{N\}}\right|>\frac{2 a_{1} \cdot 2^{N}}{d^{3 / 2} \tau}, \quad\left|U^{(N)}\right|>\frac{2 a_{1} \cdot 2^{N}}{d^{3 / 2} \tau}
$$

and by

$$
\begin{aligned}
& \mu=\min \left\{m: p \leq m \leq N, \quad \frac{\tau^{2} d^{3} p}{2^{m-p}} \leq a_{2} ;\right. \\
& \left.\quad\left|S^{\{n\}}\right| \leq \frac{2 a_{1} \cdot 2^{n}}{d^{3 / 2} \tau}, \quad\left|U^{(n)}\right| \leq \frac{2 a_{1} \cdot 2^{u m+v n}}{d^{3 / 2} \tau}, n=m, \ldots, N\right\}
\end{aligned}
$$

otherwise. Here the numbers $u, v$ are defined by (8.1). Note that the first of inequalities (8.15) is not satisfied in our conditions (see (8.6)). The same can be said about inequalities (8.31), (8.35).

Denote

$$
Y_{*, k}=Y_{k}^{[N-p]}=Z_{(k-1) \cdot 2^{p}+1}^{[N]}, \quad k=1, \ldots, 2^{N-p} .
$$

By construction and by virtue of $(6.14),(8.9)$, we have

$$
\mathbb{P}\left\{\left|Y_{*, k}\right| \leq 4 \lambda\right\}=1, \quad k=1, \ldots, 2^{N-p} .
$$

Let $i=i(j)$ be the largest integer such that $(i-1) \cdot 2^{p}+1 \leq j$. We shall denote by $V_{*}^{(n)}, n=0, \ldots, N-p$, the sum of a block of $2^{n}$ summands $Y_{*, k}$ which contains the summand $Y_{*, i}$. Let

$$
T_{*}=T_{*, i}=Y_{*, 1}+\cdots+Y_{*, i} .
$$

Recall that, by construction,

$\bigcup_{k=1}^{2^{N-p}}\left\{\left\{\widetilde{X}_{r}=Z_{r}^{[N]}, r=(k-1) \cdot 2^{p}+2, \ldots, k \cdot 2^{p}\right\} \cup\left\{Y_{*, k}=Z_{(k-1) \cdot 2^{p}+1}^{[N]}\right\}\right\}$

is a collection of random vectors, which are mutually independent. Set

$$
U_{0}^{(n)}=U^{(n)}-V_{*}^{(n-p)}, \quad n=p, \ldots, N .
$$

It is easy to see that the sum $U_{0}^{(n)}$ consists of the summands of the sum $U^{(n)}$ with indices which can not be represented in the form $r=(k-1) \cdot 2^{p}+1$ 
with integer $k$. In other words, the sum $U_{0}^{(n)}$ is the sum of contained in $U^{(n)}$ summands $Z_{r}^{[N]}$ which are chosen as $\widetilde{X}_{r}$. For the proof of this fact one should note that, by construction, the numbers $j$ and $(i-1) \cdot 2^{p}+1$ are contained in the same block of indices $\left\{m: m=(i-1) \cdot 2^{p}+1, \ldots, i \cdot 2^{p}\right\}$. Recall that, according to our construction, after the first step, when the independent random vectors $(8.20)$ are constructed, we define the rest of the random vectors $\widetilde{X}_{r}$ as the (d-dimensional) functions of the random vectors $Y_{*, k}$, $k=1, \ldots, 2^{N-p}$. Denote

$$
\begin{gathered}
X_{*, k}=\tilde{X}_{(k-1) \cdot 2^{p}+1}, \quad k=1, \ldots, 2^{N-p} \\
S_{*}=S_{*, i}=X_{*, 1}+\cdots+X_{*, i}, \quad S_{0}=S-T_{*} .
\end{gathered}
$$

By analogy with $(8.11),(8.12)$, for $s=p, \ldots, N$ we can represent the sums $S_{*}, S_{0}$ and $T_{*}$ in the form

$$
S_{0}=S_{0}^{(s)}-S_{0}^{\{s\}}, \quad T_{*}=T_{*}^{(s-p)}-T_{*}^{\{s-p\}},
$$

where

$$
\begin{aligned}
T_{*}^{(s-p)} & =T_{*, k_{s} \cdot 2 s-p}, & T_{*}^{\{s-p\}} & =T_{*}^{(s-p)}-T_{*}, \\
S_{0}^{(s)} & =S^{(s)}-T_{*}^{(s-p)}, & S_{0}^{\{s\}} & =S_{0}^{(s)}-S_{0}=S^{\{s\}}-T_{*}^{\{s-p\}},
\end{aligned}
$$

and the numbers $k_{s}$ are defined above, before the formulas (8.11), (8.12).

From the definition of numbers $i, j, k_{s}, s=0, \ldots, N$, it follows that

$$
\begin{array}{ll}
0 \leq k_{s} \cdot 2^{s}-j<2^{s}, & s=0, \ldots, N ; \\
0 \leq k_{s} \cdot 2^{s-p}-i<2^{s-p}, & s=p, \ldots, N .
\end{array}
$$

It is clear that from the relations (5.5), (8.9), (8.11), (8.12), (8.18), (8.19), (8.24) and (8.25) one can derive that, with probability one,

$$
\begin{aligned}
\left|T^{\{s\}}\right| & \leq 4 \lambda \cdot 2^{s}, & & s=0, \ldots, N \\
\left|T_{*}^{\{s-p\}}\right| & \leq 4 \lambda \cdot 2^{s-p}, & & s=p, \ldots, N .
\end{aligned}
$$

Analogously, using (8.18) and the definition of $V_{*}^{(n)}$, we see that

$$
\left|V_{*}^{(n)}\right| \leq 4 \lambda \cdot 2^{n}, \quad n=0, \ldots, N-p .
$$

Define as well

$$
\begin{aligned}
\widetilde{S}=\sum_{k=1}^{j} \widetilde{X}_{k}, \quad \delta_{j}(N) & =\left|\sum_{k=1}^{j} \widetilde{Y}_{k}-\sum_{k=1}^{j} \widetilde{X}_{k}\right|=|T-\widetilde{S}| \\
\delta_{i}(N-p) & =\left|\sum_{k=1}^{i} Y_{*, k}-\sum_{k=1}^{i} X_{*, k}\right|=\left|T_{*}-S_{*}\right| \\
\psi_{j}(N) & =\left|\sum_{k=1}^{j} \tilde{Y}_{k}-\sum_{k=1}^{j} Z_{k}^{[N]}\right|=|T-S|
\end{aligned}
$$


(see $(5.5),(5.11),(8.19),(8.22))$. It is clear that from $(8.17),(8.19),(8.20)$, $(8.22),(8.28)$ and from the definition of $i$ and $S$ it follows that

$$
S=\sum_{k=1}^{j} Z_{k}^{[N]}=S_{0}+T_{*}, \quad \widetilde{S}=S_{0}+S_{*}, \quad S-\widetilde{S}=T_{*}-S_{*} .
$$

It is easy to see that the relations (8.28), (8.29) imply

$$
\delta_{j}(N) \leq \psi_{j}(N)+\delta_{i}(N-p) .
$$

Define the random integer $\beta_{j}(N)$ by $\beta_{j}(N)=N+1$, if at least one of the following inequalities is valid:

$$
\frac{\tau^{2} d^{3} p}{2^{N-p}}>a_{2}, \quad\left|V^{(N)}\right|>\frac{4 a_{1} \cdot 2^{N}}{d^{3 / 2} \tau}
$$

and by

$$
\begin{aligned}
\beta_{j}(N)=\min \{m: p \leq & m \leq N, \quad \frac{\tau^{2} d^{3} p}{2^{m-p}} \leq a_{2}, \\
& \left.\left|V^{(n)}\right| \leq \frac{4 a_{1} \cdot 2^{u m+v n}}{d^{3 / 2} \tau}, n=m, \ldots, N\right\}
\end{aligned}
$$

otherwise.

Similarly, taking into account the relations (8.7), we define the random integer $\beta_{i}(N-p)$ by the equality $\beta_{i}(N-p)=N-p+1$, if at least one of the following inequalities is valid:

$$
\frac{\tau^{2} d^{3} p}{2^{N-2 p}}>a_{2}, \quad\left|V_{*}^{(N-p)}\right|>\frac{4 a_{1} \cdot 2^{N-p}}{d^{3 / 2} \tau}
$$

and by

$$
\begin{aligned}
\beta_{i}(N-p)=\min \{m: p & \leq m \leq N-p, \quad \frac{\tau^{2} d^{3} p}{2^{m-p}} \leq a_{2}, \\
& \left.\left|V_{*}^{(n)}\right| \leq \frac{4 a_{1} \cdot 2^{u m+v n}}{d^{3 / 2} \tau}, n=m, \ldots, N-p\right\}
\end{aligned}
$$

otherwise.

Finally, define the random integer $\nu=\nu_{j}$ by $\nu=N+1$, if at least one of the following inequalities is valid:

$$
\frac{\tau^{2} d^{3} p}{2^{N-p}}>a_{2}, \quad\left|U_{0}^{(N)}\right|>\frac{a_{1} \cdot 2^{N}}{d^{3 / 2} \tau}, \quad\left|S_{0}^{\{N\}}\right|>\frac{a_{1} \cdot 2^{N}}{d^{3 / 2} \tau}
$$

and by

$$
\begin{aligned}
\nu= & \min \left\{m: p \leq m \leq N, \quad \frac{\tau^{2} d^{3} p}{2^{m-p}} \leq a_{2} ;\right. \\
& \left.\left|S_{0}^{\{n\}}\right| \leq \frac{a_{1} \cdot 2^{n}}{d^{3 / 2} \tau}, \quad\left|U_{0}^{(n)}\right| \leq \frac{a_{1} \cdot 2^{u m+v n}}{d^{3 / 2} \tau}, n=m, \ldots, N\right\}
\end{aligned}
$$


otherwise.

Put now

$$
\begin{gathered}
\rho_{j}(N)=5 \lambda \cdot 2^{\beta_{j}(N)}, \quad \rho_{i}(N-p)=5 \lambda \cdot 2^{\beta_{i}(N-p),}, \\
\gamma_{j}(N)=\left\{\begin{array}{cl}
10 \lambda \cdot 2^{\nu}, & \text { if } \quad \nu \leq N, \\
U+22 \lambda \cdot 2^{N}, & \text { if } \quad \nu=N+1,
\end{array}\right.
\end{gathered}
$$

where

$$
U=\left|U_{0}^{(N)}\right|+\left|S_{0}^{\{N\}}\right|
$$

Lemma 8.1. The following inequalities are valid:

$$
\delta_{j}(N)+\rho_{j}(N) \leq \delta_{i}(N-p)+\rho_{i}(N-p)+\gamma_{j}(N)
$$

and, for each $\varepsilon>0$,

$\mathbb{E} \exp \left(\varepsilon\left(\delta_{j}(N)+\rho_{j}(N)\right)\right) \leq \mathbb{E} \exp \left(\varepsilon\left(\delta_{i}(N-p)+\rho_{i}(N-p)\right)\right) \mathbb{E} \exp \left(\varepsilon \gamma_{j}(N)\right)$.

Proof. Assume that $\nu \leq N$ and, taking into account (8.7), define the integer valued random variables

$$
\beta=\beta_{i}(N-p)-1 \leq N-p, \quad \phi=\max \{\beta, \nu\} \leq N .
$$

The relations (8.1), (8.4), (8.26) imply

$$
\left|T_{*}^{\{s-p\}}\right| \leq \frac{a_{1} \cdot 2^{s}}{d^{3 / 2} \tau}, \quad s=p, \ldots, N .
$$

Using (8.24), (8.36), (8.42), (8.43), we obtain

$$
\left|S^{\{n\}}\right| \leq\left|S_{0}^{\{n\}}\right|+\left|T_{*}^{\{n-p\}}\right| \leq \frac{2 a_{1} \cdot 2^{n}}{d^{3 / 2} \tau}, \quad n=\phi, \ldots, N,
$$

(here and below we use that (8.36), (8.42) imply the inequality $\phi \geq p$ ). By virtue of $(8.1),(8.4),(8.27),(8.42)$, for $n=\beta, \ldots, \beta+p, n \geq p$ we have

$$
\left|V_{*}^{(n-p)}\right| \leq 4 \lambda \cdot 2^{n-p} \leq \frac{a_{1} \cdot 2^{u \beta+v n}}{d^{3 / 2} \tau} \leq \frac{a_{1} \cdot 2^{u \phi+v n}}{d^{3 / 2} \tau} .
$$

If $n=\beta+p+1, \ldots, N$, then, applying (8.1), (8.2), (8.4), (8.34), (8.42), we obtain

$$
\left|V_{*}^{(n-p)}\right| \leq \frac{4 a_{1} \cdot 2^{u(\beta+1)+v(n-p)}}{d^{3 / 2} \tau} \leq \frac{a_{1} \cdot 2^{u \phi+v n}}{d^{3 / 2} \tau}
$$

(we use that $p \geq 4,(8.1),(8.2)$ imply $2^{v p} \geq 2^{5 p / 8}>4 \cdot 2^{3 / 8} \geq 4 \cdot 2^{u}$ ). From $(8.21),(8.36),(8.42),(8.45),(8.46)$ it follows that

$$
\begin{aligned}
& \left|U^{(n)}\right| \leq\left|U_{0}^{(n)}\right|+\left|V_{*}^{(n-p)}\right| \leq \frac{a_{1} \cdot 2^{u \phi+v n}}{d^{3 / 2} \tau}+\frac{a_{1} \cdot 2^{u \phi+v n}}{d^{3 / 2} \tau} \\
& =\frac{2 a_{1} \cdot 2^{u \phi+v n}}{d^{3 / 2} \tau}, \quad n=\phi, \ldots, N \text {. }
\end{aligned}
$$


Taking into account $(8.16),(8.36),(8.42),(8.44),(8.47)$, we see that

$$
\mu \leq \phi \leq N
$$

It is clear that

$$
U^{(m)}=\frac{1}{2} U^{(m+1)}+\frac{b_{m+1}}{2} \widetilde{U}^{(m+1)}, \quad m=0, \ldots, N-1,
$$

where the factors $b_{m+1}=b_{m+1}(j)$ can be equal either to 1 or to -1 (see (5.10)). From (8.49) one can easily derive by induction that the following representation is valid:

$$
\begin{gathered}
U^{(m)}=\frac{U^{(N)}}{2^{N-m}}+\sum_{\substack{k=m+1 \\
b_{k} \mid}=1, \quad k=1, \ldots, N} \frac{b_{k} \widetilde{U}^{(k)}}{2^{k-m}}, \quad m=0, \ldots, N-1,
\end{gathered}
$$

It is evident that we can write the analogous equality with change of $U$ by $V$.

Suppose now that an integer $n$ satisfies the inequalities

$$
\mu \leq n \leq N
$$

Then, taking into account (8.1)-(8.4), (8.16), (8.51), we see that

$$
\tau^{2} d^{3} \leq a_{2} \cdot 2^{\mu} \leq a_{1}^{2} \cdot 2^{2 u \mu+(2 v-1) n} \leq a_{1}^{2} \cdot 2^{2 u \mu+(2 v-1) N} .
$$

Using $(8.1),(8.3),(8.16),(8.51)$, we obtain the inequalities

$$
\left|U^{(n)}\right| \leq \frac{2 a_{1} \cdot 2^{u \mu+v n}}{d^{3 / 2} \tau} \leq \frac{c_{67} \cdot 2^{n}}{d^{3 / 2} \tau} .
$$

Taking here $n=N$ and using the relations $(8.1)-(8.3),(8.16),(8.51)-(8.53)$ and Lemma $7.2(\mathrm{~b})$, we obtain

$$
\begin{aligned}
\left|U^{(N)}-V^{(N)}\right| & \leq c_{47} d^{2} \tau^{2} \cdot 2^{-N / 2}\left(1+2^{-3 N / 2}\left|U^{(N)}\right|^{3}\right) \\
& \leq c_{66} d^{2} \tau^{2}\left(2^{-N / 2}+\frac{8 a_{1}^{3} \cdot 2^{3 u \mu-w N}}{d^{9 / 2} \tau^{3}}\right) \\
& \leq \frac{9 a_{1}^{3} c_{66} \cdot 2^{3 u \mu-w N}}{d^{5 / 2} \tau} \leq \frac{a_{1} \cdot 2^{3 u \mu-w N}}{14 d^{5 / 2} \tau}
\end{aligned}
$$

From (8.1), (8.53), (8.54) it follows that

$$
\left|V^{(N)}\right| \leq\left|U^{(N)}\right|+\left|V^{(N)}-U^{(N)}\right| \leq \frac{4 a_{1} \cdot 2^{u \mu+v N}}{d^{3 / 2} \tau} .
$$

Analogously, if we suppose that

$$
\mu \leq n<N
$$


then from $(8.1)-(8.3),(8.16),(8.49),(8.56)$ one can easily derive that

$$
\left|\tilde{U}^{(n+1)}\right|=\left|U^{(n+1)}-2 U^{(n)}\right| \leq \frac{2\left(1+2^{3 / 8}\right) a_{1} \cdot 2^{u \mu+v(n+1)}}{d^{3 / 2} \tau} \leq \frac{c_{67} \cdot 2^{n+1}}{d^{3 / 2} \tau} .
$$

Using the induction with respect to $n$, we shall prove that, if $\mu<N$, then, for $n=N-1, N-2, \ldots, \mu$,

$$
\begin{aligned}
\left|\tilde{U}^{(n+1)}-\tilde{V}^{(n+1)}\right| & \leq \frac{2 a_{1} \cdot 2^{3 u \mu-w(n+1)}}{d^{5 / 2} \tau}, \\
\left|U^{(n)}-V^{(n)}\right| & \leq \frac{2 a_{1} \cdot 2^{3 u \mu-w n}}{d^{5 / 2} \tau}, \\
\left|V^{(n)}\right| & \leq \frac{4 a_{1} \cdot 2^{u \mu+v n}}{d^{3 / 2} \tau} .
\end{aligned}
$$

For $n=N$ the relations $(8.59),(8.60)$ are already proved (see $(8.54),(8.55)$ ).

Assume that the inequalities $(8.59),(8.60)$ are valid for $n=s+1, \ldots, N$ and the inequality (8.58) holds for $n=s+1, \ldots, N-1, \mu \leq s \leq N-1$. It suffices to prove the relations $(8.58)-(8.60)$ for $n=s$.

Using (8.1)-(8.3), (8.16), (8.52), (8.53), (8.56), (8.57), (8.59), (8.60) and applying Lemma $7.2(\mathrm{a})$, we obtain that in this case

$$
\begin{aligned}
& \left|\tilde{U}^{(s+1)}-\tilde{V}^{(s+1)}\right| \\
& \leq \frac{c_{44} d^{2} \tau^{2}}{2^{(s+1) / 2}}\left(1+\frac{\max \left\{\left|U^{(s+1)}\right|^{3},\left|\widetilde{U}^{(s+1)}\right|^{3}\right\}}{2^{3(s+1) / 2}}\right)+\frac{\left|U^{(s+1)}-V^{(s+1)}\right|}{8} \\
& \leq c_{66} d^{2} \tau^{2}\left(2^{-(s+1) / 2}+\frac{125 a_{1}^{3} \cdot 2^{3 u \mu-w(s+1)}}{d^{9 / 2} \tau^{3}}\right)+\frac{a_{1} \cdot 2^{3 u \mu-w(s+1)}}{4 d^{5 / 2} \tau} \\
& \leq \frac{2 a_{1} \cdot 2^{3 u \mu-w(s+1)}}{d^{5 / 2} \tau} .
\end{aligned}
$$

The fulfilment of the conditions of Lemma 7.2 can be easily deduced from (8.1), (8.16), (8.53), (8.57), (8.60) (with $n=s+1$ ) and from the definitions of $a_{1}, c_{67}$ (see $\left.(8.3)\right)$.

From $(8.1),(8.2),(8.50),(8.53),(8.54),(8.58)$ (with $n=s, \ldots, N-1$ ) it follows that

$$
\left|U^{(s)}-V^{(s)}\right| \leq \frac{\left|U^{(N)}-V^{(N)}\right|}{2^{N-s}}+\sum_{k=s+1}^{N} \frac{\left|\widetilde{U}^{(k)}-\widetilde{V}^{(k)}\right|}{2^{k-s}} \leq \frac{2 a_{1} \cdot 2^{3 u \mu-w s}}{d^{5 / 2} \tau}
$$

and, consequently,

$$
\begin{aligned}
\left|V^{(s)}\right| & \leq\left|U^{(s)}\right|+\left|U^{(s)}-V^{(s)}\right| \\
& \leq \frac{2 a_{1} \cdot 2^{u \mu+v s}}{d^{3 / 2} \tau}+\frac{2 a_{1} \cdot 2^{3 u \mu-w s}}{d^{5 / 2} \tau} \leq \frac{4 a_{1} \cdot 2^{u \mu+v s}}{d^{3 / 2} \tau} .
\end{aligned}
$$

This completes the proof of $(8.58)-(8.60)$ for $n=s$.

It is evident that from $(8.16),(8.32),(8.55),(8.60)$ it follows that

$$
\beta_{j}(N) \leq \mu \text {. }
$$


It is clear that the relations $(8.42),(8.48),(8.61)$ imply

$$
2^{\beta_{j}(N)}+2^{\mu} \leq 2 \cdot 2^{\mu} \leq 2 \cdot 2^{\phi} \leq 2^{\beta_{i}(N-p)}+2 \cdot 2^{\nu} .
$$

Using (8.2), (8.3), (8.14), (8.16), (8.26), (8.28), (8.54), (8.58) and taking into account that $\lambda \geq 1, \tau \geq 1$, we see that

$$
\begin{aligned}
\psi_{j}(N) & \leq \frac{a_{1} \cdot 2^{3 u \mu-w N}}{14 d^{5 / 2} \tau}+\sum_{n=\mu+1}^{N} \frac{2 a_{1} \cdot 2^{3 u \mu-w n}}{d^{5 / 2} \tau}+\frac{2 a_{1} \cdot 2^{\mu}}{d^{3 / 2} \tau}+4 \lambda \cdot 2^{\mu} \\
& \leq\left(2\left(1-2^{-w}\right)^{-1} a_{1}+4 \lambda\right) \cdot 2^{\mu} \leq 5 \lambda \cdot 2^{\mu}
\end{aligned}
$$

The relations $(8.30),(8.37),(8.62),(8.63)$ imply

$$
\begin{aligned}
\delta_{j}(N)+\rho_{j}(N) & \leq \delta_{i}(N-p)+5 \lambda\left(2^{\mu}+2^{\beta_{j}(N)}\right) \\
& \leq \delta_{i}(N-p)+\rho_{i}(N-p)+10 \lambda \cdot 2^{\nu} .
\end{aligned}
$$

From (8.38), (8.64) it is easy to derive the inequality (8.40) in the case $\nu \leq N$.

Let now $\nu=N+1$. From $(8.12),(8.21)$ it follows that

$$
S^{(N)}=U^{(N)}=U_{0}^{(N)}+V_{*}^{(N-p)} .
$$

Using (8.11), (8.28), we see that

$$
\psi_{j}(N) \leq\left|S^{(N)}\right|+\left|S^{\{N\}}\right|+|T| .
$$

By virtue of (8.24) we have

$$
\left|S^{\{N\}}\right| \leq\left|S_{0}^{\{N\}}\right|+\left|T_{*}^{\{N-p\}}\right| .
$$

It is not hard to see that $(8.10),(8.26),(8.27),(8.39),(8.65)-(8.67)$ imply

$$
\psi_{j}(N) \leq U+12 \lambda \cdot 2^{N}
$$

From (8.37) and from definition of $\beta_{j}(N)$ it follows that $\beta_{j}(N) \leq N+1$ and

$$
\rho_{j}(N) \leq 10 \lambda \cdot 2^{N}
$$

The relations $(8.30),(8.38),(8.68),(8.69)$ imply

$$
\delta_{j}(N)+\rho_{j}(N) \leq \delta_{i}(N-p)+U+22 \lambda \cdot 2^{N}=\delta_{i}(N-p)+\gamma_{j}(N) .
$$

Thus, the inequality (8.40) is proved in the case $\nu=N+1$.

By construction (see $(8.19)-(8.24),(8.28),(8.33)-(8.38)), \gamma_{j}(N)$ is a function of

$$
\bigcup_{k=1}^{2^{N-p}}\left\{\tilde{X}_{r}=Z_{r}^{[N]}, r=(k-1) \cdot 2^{p}+2, \ldots, k \cdot 2^{p}\right\}
$$


and the random variables $\delta_{i}(N-p), \rho_{i}(N-p)$ are functions of

$$
\left\{Y_{*, k}=Z_{(k-1) \cdot 2^{p}+1}^{[N]}, k=1, \ldots, 2^{N-p}\right\} .
$$

The random variables $\gamma_{j}(N)$ and $\delta_{i}(N-p)+\rho_{i}(N-p)$ are independent, since the sets $(8.71),(8.72)$ are disjoint sets consisting of mutually independent vectors (see (8.20)), Therefore, the inequality (8.41) follows from (8.40) and the proof of Lemma 8.1 is completed.

In this section we shall denote by $\varepsilon$ the quantity (see (8.3))

$$
\varepsilon=\frac{a_{1}^{2}}{512 \lambda d^{3} \tau^{2}} \leq \frac{a_{1}}{16 d^{3 / 2} \tau}
$$

Lemma 8.2. There exists a positive quantity $a_{3}$ depending only on $v$ and such that the following inequality is valid:

$$
\mathbb{E} \exp \left(\varepsilon \gamma_{j}(N)\right) \leq \exp \left(a_{3} p\left(\lambda d^{3 / 2} \tau\right)^{1 / v}\right)
$$

Proof. Denote by

$$
\zeta=1_{\{\nu=N+1\}}
$$

the indicator function of the event $\{\nu=N+1\}$ (see (8.35)). Using (8.38) and the Hölder inequality, we obtain

$$
\mathbb{E} \zeta \exp \left(\varepsilon \gamma_{j}(N)\right) \leq\left(\mathbb{E} \zeta^{2}\right)^{1 / 2} \exp \left(22 \varepsilon \lambda \cdot 2^{N}\right)\left(\mathbb{E} e^{2 \varepsilon U}\right)^{1 / 2}
$$

From the definitions of $U_{0}^{(n)}, S_{0}^{\{n\}}$ it follows that $U_{0}^{(N)}, S_{0}^{\{N\}}$ are sums of not more than $2^{N}$ independent random vectors $\widetilde{X}_{k}$ with $\mathcal{L}\left(\widetilde{X}_{k}\right) \in \mathcal{A}_{d}(\tau)$, $\operatorname{cov} \widetilde{X}_{k}=I$ (see $\left.(8.21),(8.24)\right)$. Therefore, the relations $(8.3),(8.6),(8.35)$, (8.75) and Lemma 2.11 imply

$$
\begin{aligned}
\mathbb{E} \zeta^{2} & \leq \mathbb{P}\left\{\left|U_{0}^{(N)}\right|>\frac{a_{1} \cdot 2^{N}}{d^{3 / 2} \tau}\right\}+\mathbb{P}\left\{\left|S_{0}^{\{N\}}\right|>\frac{a_{1} \cdot 2^{N}}{d^{3 / 2} \tau}\right\} \\
& \leq 4 \boldsymbol{d} \exp \left(-\min \left\{\frac{a_{1}^{2} \cdot 2^{N}}{4 \boldsymbol{d}^{3} \tau^{2}}, \frac{a_{1} \cdot 2^{N}}{4 \boldsymbol{d}^{3 / 2} \tau^{2}}\right\}\right) \\
& =4 \boldsymbol{d} \exp \left(-\frac{a_{1}^{2} \cdot 2^{N}}{4 \boldsymbol{d}^{3} \tau^{2}}\right) .
\end{aligned}
$$

It is clear that (see (8.39))

$$
\mathbb{E} e^{2 \varepsilon U} \leq\left(\mathbb{E} \exp \left(4 \varepsilon\left|U_{0}^{(N)}\right|\right) \mathbb{E} \exp \left(4 \varepsilon\left|S_{0}^{\{N\}}\right|\right)\right)^{1 / 2}
$$

By virtue of $(8.3),(8.73)$ we have

$$
4 \varepsilon \tau<1 .
$$

Taking into account (8.79) and applying Lemma 2.11 with $B^{2} \leq 2^{N}$, $h=4 \varepsilon$, we see that

$$
\begin{aligned}
& \mathbb{E} \exp \left(4 \varepsilon\left|U_{0}^{(N)}\right|\right) \leq 2 d \exp \left(16 \varepsilon^{2} \cdot 2^{N}\right), \\
& \mathbb{E} \exp \left(4 \varepsilon\left|S_{0}^{\{N\}}\right|\right) \leq 2 d \exp \left(16 \varepsilon^{2} \cdot 2^{N}\right) .
\end{aligned}
$$


From $(8.3),(8.4),(8.6),(8.73),(8.76)-(8.78),(8.80)$ it follows that

$$
\begin{aligned}
\mathbb{E} \zeta \exp \left(\varepsilon \gamma_{j}(N)\right) & \leq 2 \sqrt{2} d \exp \left(-\frac{a_{1}^{2} \cdot 2^{N}}{8 d^{3} \tau^{2}}+22 \varepsilon \lambda \cdot 2^{N}+8 \varepsilon^{2} \cdot 2^{N}\right) \\
& \leq 2 \sqrt{2} d \exp \left(-\frac{a_{1}^{2} \cdot 2^{N}}{32 d^{3} \tau^{2}}\right) \leq 2 \sqrt{2} d \exp \left(-\frac{p \cdot 2^{p}}{32}\right) \\
& \leq 2 \sqrt{2} d \exp \left(-\frac{d^{3 / 2} \tau \lambda}{8 a_{1}}\right) \leq c
\end{aligned}
$$

Denote now by $m_{0}$ the minimal integer such that

$$
\frac{\tau^{2} d^{3} p}{2^{m_{0}-p}}<a_{2}
$$

It is evident that (8.3), (8.5)-(8.7), (8.82) imply $p \leq m_{0}=M+1 \leq N$. Using the definition of the random variable $\nu$, we see that $\mathbb{P}\{\nu=m+1\}$, $m=m_{0}, \ldots, N-1$, can be estimated from above by

$$
\sum_{n=m}^{N}\left(\mathbb{P}\left\{\left|U_{0}^{(n)}\right| \geq \frac{a_{1} \cdot 2^{u m+v n}}{d^{3 / 2} \tau}\right\}+\mathbb{P}\left\{\left|S_{0}^{\{n\}}\right| \geq \frac{a_{1} \cdot 2^{n}}{d^{3 / 2} \tau}\right\}\right) .
$$

It is clear that the vectors $U_{0}^{(n)}, S_{0}^{\{n\}}$ are sums of not more than $2^{n}$ independent random vectors $\widetilde{X}_{k}$ with $\mathcal{L}\left(\widetilde{X}_{k}\right) \in \mathcal{A}_{d}(\tau)$, cov $\widetilde{X}_{k}=I$ (see (8.21) and (8.24)). Therefore, for $m \geq m_{0}$, the relations (8.1), (8.3), (8.82) and Lemma 2.11 imply

$$
\begin{aligned}
\mathbb{P}\left\{\left|U_{0}^{(n)}\right|\right. & \left.\geq \frac{a_{1} \cdot 2^{u m+v n}}{d^{3 / 2} \tau}\right\} \\
& \leq 2 d \exp \left(-\min \left\{\frac{a_{1}^{2} \cdot 2^{2 u m+2 v n}}{4 d^{3} \tau^{2} \cdot 2^{n}}, \frac{a_{1} \cdot 2^{u m+v n}}{4 d^{3 / 2} \tau^{2}}\right\}\right) \\
& =2 d \exp \left(-\frac{a_{1}^{2} \cdot 2^{2 u m+(2 v-1) n}}{4 d^{3} \tau^{2}}\right), \quad n=m, \ldots, N,
\end{aligned}
$$

and, analogously,

$$
\mathbb{P}\left\{\left|S_{0}^{\{n\}}\right| \geq \frac{a_{1} \cdot 2^{n}}{d^{3 / 2} \tau}\right\} \leq 2 d \exp \left(-\frac{a_{1}^{2} \cdot 2^{n}}{4 d^{3} \tau^{2}}\right), \quad n=m, \ldots, N .
$$

Taking into account $(8.38),(8.75)$, we see that

$$
\mathbb{E}(1-\zeta) \exp \left(\varepsilon \gamma_{j}(N)\right) \leq \exp \left(10 \lambda \varepsilon \cdot 2^{m_{0}}\right)+\sum_{m=m_{0}+1}^{N} e^{10 \lambda \varepsilon \cdot 2^{m}} \mathbb{P}\{\nu=m\}
$$

From (8.1), (8.3), (8.73), (8.82) and from the definition of $p$ (see (8.4)) it follows that

$$
10 \lambda \varepsilon \cdot 2^{m_{0}} \leq \frac{10 a_{1}^{2}}{512 d^{3} \tau^{2}} \cdot \frac{d^{3} \tau^{2} p \cdot 2^{p+1}}{a_{2}} \leq a p \cdot 2^{p} \leq a p\left(\lambda d^{3 / 2} \tau\right)^{1 / v} .
$$


Using (8.1)-(8.4), (8.73), (8.82)-(8.85), we obtain

$$
\begin{aligned}
& \sum_{m=m_{0}+1}^{N} e^{10 \lambda \varepsilon \cdot 2^{m}} \mathbb{P}\{\nu=m\} \\
& \quad \leq \sum_{m=m_{0}+1}^{N} \sum_{n=m-1}^{N} 4 d \exp \left(\frac{10 a_{1}^{2} \cdot 2^{m}}{512 d^{3} \tau^{2}}-\frac{a_{1}^{2} \cdot 2^{2 u m+(2 v-1) n}}{8 d^{3} \tau^{2}}\right) \\
& \quad \leq 4 d \sum_{m=m_{0}+1 n=m-1}^{N} \sum_{m=m_{0}+1}^{N} \exp \left(-\frac{a_{1}^{2} \cdot 2^{2 u m+(2 v-1) n}}{16 d^{3} \tau^{2}}\right) \\
& \quad \leq 4 d \sum_{m=}^{\infty} c L\left(a_{1}^{-1} d \tau\right) \exp \left(-\frac{a_{1}^{2} \cdot 2^{m}}{32 d^{3} \tau^{2}}\right) \\
& \quad \leq c d L^{2}\left(a_{1}^{-1} d \tau\right) \exp \left(-\frac{a_{1}^{2} \cdot 2^{m_{0}}}{16 d^{3} \tau^{2}}\right) \\
& \quad \leq c d L^{2}\left(a_{1}^{-1} d \tau\right) \exp \left(-\frac{p \cdot 2^{p}}{16}\right) \\
& \quad \leq c d L^{2}\left(a_{1}^{-1} d \tau\right) \exp \left(-\frac{d^{3 / 2} \tau \lambda}{4 a_{1}}\right) \leq c .
\end{aligned}
$$

The inequality (8.74) follows from (8.81), (8.86)-(8.88).

Lemma 8.3. Assume now (instead of (8.6)) that $N=M$. Then there exists a positive quantity $a_{4}$ depending only on $v$ and such that the following inequality is valid:

$$
\mathbb{E} \exp \left(\varepsilon\left(\rho_{j}(M)+\delta_{j}(M)\right)\right) \leq \exp \left(a_{4} p\left(\lambda d^{3 / 2} \tau\right)^{1 / v}\right), \quad j=1, \ldots, 2^{M} .
$$

Proof. Similarly to (8.69), one can verify that $\rho_{j}(M) \leq 10 \lambda \cdot 2^{M}$. Therefore, using (8.28), we obtain

$$
\mathbb{E} \exp \left(\varepsilon\left(\rho_{j}(M)+\delta_{j}(M)\right)\right) \leq \exp \left(10 \varepsilon \lambda \cdot 2^{M}\right) \mathbb{E} e^{\varepsilon W},
$$

where $W=|\widetilde{S}|+|T|$. From the definitions of $\widetilde{S}, T=T_{j}$ (see (5.5) and (8.28)) it follows that they are sums of not more than $2^{M}$ independent random vectors $\widetilde{X}_{k}$ and $Y_{k}^{[M]}$ respectively. By analogy with $(8.10)$, we have $\mathbb{P}\left\{|T| \leq 4 \lambda \cdot 2^{M}\right\}=1$. Hence,

$$
\mathbb{E} e^{\epsilon W} \leq \mathbb{E} e^{\varepsilon|\widetilde{S}|} \exp \left(4 \varepsilon \lambda \cdot 2^{M}\right) .
$$

Recall that $\operatorname{cov} \widetilde{X}_{k}=I, \mathcal{L}\left(\tilde{X}_{k}\right) \in \mathcal{A}_{d}(\tau)$. Taking into account (8.79) and applying Lemma 2.11 with $B^{2} \leq 2^{M}, h=\varepsilon$, we see that

$$
\mathbb{E} e^{\varepsilon|\widetilde{S}|} \leq 2 d \exp \left(\varepsilon^{2} \cdot 2^{M}\right) \text {. }
$$

From $(8.1),(8.3),(8.5),(8.73),(8.90)-(8.92)$ and from the definition of $p$ (see $(8.4)$ ) it follows that

$$
\begin{aligned}
\mathbb{E} \exp \left(\varepsilon\left(\rho_{j}(M)+\delta_{j}(M)\right)\right) & \leq 2 d \exp \left(14 \varepsilon \lambda \cdot 2^{M}+\varepsilon^{2} \cdot 2^{M}\right) \\
& \leq 2 d \exp \left(\frac{15 a_{1}^{2} \cdot 2^{M}}{512 d^{3} \tau^{2}}\right) \leq 2 d \exp \left(a p \cdot 2^{p}\right) \\
& \leq 2 d \exp \left(a p\left(\lambda d^{3 / 2} \tau\right)^{1 / v}\right) .
\end{aligned}
$$


It is evident that $(8.1),(8.4),(8.93)$ implies (8.89).

Denote

$$
\begin{aligned}
b(N) & =\max _{1 \leq m \leq 2^{N}} \mathbb{E} \exp \left(\varepsilon\left(\delta_{m}(N)+\rho_{m}(N)\right)\right), \\
b(N-p) & =\max _{1 \leq m \leq 2^{N-p}} \mathbb{E} \exp \left(\varepsilon\left(\delta_{m}(N-p)+\rho_{m}(N-p)\right)\right) \\
r(N) & =\max _{1 \leq m \leq 2^{N}} \mathbb{E} \exp \left(\varepsilon \gamma_{m}(N)\right) .
\end{aligned}
$$

One can easily verify that the definitions of $b(N), b(N-p)$ are good coordinated: if we begin with $2^{N_{0}}$ vectors, where $N_{0}=N-p$, then we have $b\left(N_{0}\right)=b(N-p)$ (see $\left.(8.17),(8.28)\right)$. Since $j, 1 \leq j \leq 2^{N}$, is arbitrary, we can easily derive from $(8.41),(8.94)$ and Lemma 8.2 that

$$
b(N) \leq b(N-p) r(N) \leq b(N-p) \exp \left(a_{3} p\left(\lambda d^{3 / 2} \tau\right)^{1 / v}\right) .
$$

Continuing this procedure and taking into account $(8.5)-(8.7)$, we obtain

$$
b(N) \leq b(M) \exp \left(a_{3}(N-M)\left(\lambda d^{3 / 2} \tau\right)^{1 / v}\right) .
$$

From $(8.7),(8.89),(8.94),(8.96)$ it follows that

$$
b(N) \leq \exp \left(a_{5} N\left(\lambda d^{3 / 2} \tau\right)^{1 / v}\right)
$$

with $a_{5}=\max \left\{a_{3}, a_{4}\right\}$. Using (8.28), (8.94), (8.97), we obtain

$$
\begin{aligned}
\mathbb{E} \exp (\varepsilon \Delta(\tilde{X}, \tilde{Y})) & \leq \sum_{m=1}^{2^{N}} \mathbb{E} \exp \left(\varepsilon \delta_{m}(N)\right) \\
& \leq \sum_{m=1}^{2^{N}} \mathbb{E} \exp \left(\varepsilon\left(\delta_{m}(N)+\rho_{m}(N)\right)\right) \\
& \leq 2^{N} \exp \left(a_{5} N\left(\lambda d^{3 / 2} \tau\right)^{1 / v}\right) \\
& \leq \exp \left(a N\left(\lambda d^{3 / 2} \tau\right)^{1 / v}\right)
\end{aligned}
$$

Assume now, instead of (8.6), that $N \leq M$ and suppose that the vectors $\tilde{X}_{k}, k=1, \ldots, 2^{M}$, are constructed as functions of the vectors $\tilde{Y}_{k}$, $k=1, \ldots, 2^{M}$, in the same way as $X_{k}^{*}=\widetilde{X}_{(k-1) \cdot 2^{N-M}+1}, k=1, \ldots, 2^{M}$, were constructed as functions of $Y_{k}^{*}=Z_{(k-1) \cdot 2^{N-M}+1}^{[M+p]}, k=1, \ldots, 2^{M}$. For $k=2^{N}+1, \ldots, 2^{M}$ we can take $\mathcal{L}\left(\widetilde{X}_{k}\right)=\mathcal{L}\left(\widetilde{X}_{1}\right), \mathcal{L}\left(\widetilde{Y}_{k}\right)=\mathcal{L}\left(\widetilde{Y}_{1}\right)$. Now, if we denote

$$
\Delta_{N}(\tilde{X}, \tilde{Y})=\max _{1 \leq j \leq 2^{N}}\left|\sum_{k=1}^{j} \tilde{Y}_{k}-\sum_{k=1}^{j} \tilde{X}_{k}\right|,
$$

then, using (8.28), (8.89), (8.94), (8.99), we obtain

$$
\begin{aligned}
\mathbb{E} \exp \left(\varepsilon \Delta_{N}(\tilde{X}, \tilde{Y})\right) & \leq \sum_{m=1}^{2^{N}} \mathbb{E} \exp \left(\varepsilon \delta_{m}(M)\right) \leq 2^{N} \exp \left(a_{4} p\left(\lambda d^{3 / 2} \tau\right)^{1 / v}\right) \\
& \leq \exp \left(a(N+p)\left(\lambda d^{3 / 2} \tau\right)^{1 / v}\right)
\end{aligned}
$$


Proof of Theorem 1.5. Without loss of generality we shall suppose that $0<\alpha \leq 1 / 5$ and $n=2^{N}$, with $N \geq 1$. Assume that independent Gaussian vectors $Y_{1}, \ldots, Y_{2^{N}}$ are already constructed. According to Theorem 6.4 , we can construct the independent random vectors $\tilde{Y}_{1}, \ldots, \widetilde{Y}_{2^{N}}$ so that $\mathcal{L}\left(\widetilde{Y}_{k}\right)=$ $\Psi\left(\widetilde{\eta}_{k}\right), k=1, \ldots, n$, and

$$
\mathbb{E} \exp \left(\frac{c_{16} \Delta(\tilde{Y}, Y)}{d^{3 / 2} \tau}\right) \leq \exp \left(c_{17} N L(d)\right) .
$$

Then we construct the vectors $\widetilde{X}_{1}, \ldots, \widetilde{X}_{2^{N}}$ as functions of $\widetilde{Y}_{1}, \ldots, \widetilde{Y}_{2^{N}}$ as it was described above in this section.

Taking $v=\frac{2}{3+\alpha}$, we see that $v$ satisfies (8.1) and

$$
v^{-1}=\frac{3+\alpha}{2}
$$

The relations $(8.1),(8.3),(8.102)$ show that $a, a_{1}, \ldots, a_{5}$ can be considered as quantities depending only on $\alpha$.

Let $M$ be the maximal integer satisfying the inequality (8.5) with $a_{2}, p$ defined by $(8.3),(8.4)$. Define $N$ as the minimal integer such that $n \leq 2^{N}$, if $n \leq 2^{M}$, and as the minimal integer satisfying the relations $n \leq 2^{N}$ and (8.6), if $n>2^{M}$. In both cases we have

$$
N \leq c \log n+p .
$$

It is evident that from $(8.1),(8.4),(8.102)$ it follows that

$$
p \leq a_{6} L(\lambda d \tau) \leq a_{7}\left(\lambda d^{3 / 2} \tau\right)^{\alpha / 2}
$$

where $a_{6}, a_{7}$ depend only on $\alpha$. The statement of Theorem 1.5 follows now from $(8.73),(8.98)-(8.104)$.

\section{Proofs of Theorems 1.3 And 1.6}

A complication of the formulation of Theorem 9.1 below in comparison with that of Theorem 1.5 is necessary to make more precise the dependence of the denominators of the fractions in the left-hand sides of inequalities (1.4), (1.7) on $\tau$.

Theorem 9.1. Suppose that $\tau_{1} \geq \tau_{2} \geq 1, \tau_{3} \geq \tau_{2}, \lambda \geq 1, \alpha>0$ and $\xi_{1}, \ldots, \xi_{n}$ are independent random vectors with $\mathcal{L}\left(\xi_{k}\right) \in \mathcal{A}_{d}\left(\tau_{1}\right), \mathbb{E} \xi_{k}=0$, $\operatorname{cov} \xi_{k}=I, k=1, \ldots, n$. Let $n=2^{N} \geq \tau_{1}^{2} \tau_{2}^{-2}$ for some integer $N$ and let the integer $M \leq N$ be defined by the relation

$$
\tau_{1}^{2} \leq 2^{M} \tau_{2}^{2}<2 \tau_{1}^{2}
$$

Denote

$$
\widetilde{\xi}_{m}=2^{-M / 2}\left(\xi_{(m-1) \cdot 2^{M}+1}+\cdots+\xi_{m \cdot 2^{M}}\right), \quad m=1, \ldots, 2^{N-M} .
$$


Assume that there exist random vectors $\widetilde{\eta}_{m}, m=\underset{\widetilde{\xi}}{1}, \ldots, 2^{N-M}$, which have the same moments of the first three orders as $\widetilde{\xi}_{m}, \mathcal{L}\left(\widetilde{\eta}_{m}\right) \in \mathcal{A}_{d}\left(\tau_{3}\right)$ and $\mathbb{P}\left\{\left|\widetilde{\eta}_{m}\right| \leq \lambda\right\}=1$. Then one can construct on a probability space a sequence of independent random vectors $X_{1}, \ldots, X_{n}$ and a corresponding sequence of independent Gaussian random vectors $Y_{1}, \ldots, Y_{n}$ so that $\mathcal{L}\left(X_{k}\right)=\mathcal{L}\left(\xi_{k}\right)$, $k=1, \ldots, n$, and

$$
\mathbb{E} \exp \left(\frac{c_{7}(\alpha) \tau_{2} \Delta(X, Y)}{\lambda d^{3} \tau_{1} \tau_{3}^{2}}\right) \leq \exp \left(c_{8}(\alpha)\left(\lambda d^{3 / 2} \tau_{3}\right)^{3 / 2+\alpha} L\left(n \tau_{2}^{2} / \tau_{1}^{2}\right)\right)
$$

where $c_{7}(\alpha), c_{8}(\alpha)$ are positive quantities depending only on $\alpha$.

Proof. It is clear that $0 \leq M \leq N$ (see (9.1)) and the random vectors $\widetilde{\xi}_{1}, \ldots, \widetilde{\xi}_{2^{N-M}}$ satisfy the conditions of Theorem 1.5 with the change of $n$ by $2^{N-M}$ and $\tau$ by $\tau_{3}$ (one should use Lemma 2.5 , the completeness of $\mathcal{A}_{d}(\tau)$ with respect to convolution and the monotonicity of $\mathcal{A}_{d}(\tau)$ with respect to $\tau$ ). Therefore, one can construct on a probability space a sequence of independent random vectors $\widetilde{X}_{1}, \ldots, \widetilde{X}_{2^{N-M}}$ and a sequence of independent Gaussian random vectors $\widetilde{Y}_{1}, \ldots, \tilde{Y}_{2^{N-M}}$ so that $\mathcal{L}\left(\widetilde{X}_{m}\right)=\mathcal{L}\left(\widetilde{\xi}_{m}\right), \mathcal{L}\left(\widetilde{Y}_{m}\right)=$ $\mathcal{N}(0, I), m=1, \ldots, 2^{N-M}$, and

$$
\mathbb{E} \exp \left(2^{M / 2} b \Delta(\tilde{X}, \tilde{Y})\right) \leq \exp \left(c_{4}(\alpha)\left(\lambda d^{3 / 2} \tau_{3}\right)^{3 / 2+\alpha} L\left(2^{N-M}\right)\right),
$$

where

$$
b=\frac{c_{3}(\alpha)}{\lambda d^{3} \tau_{3}^{2} \cdot 2^{M / 2}}
$$

and $c_{3}(\alpha), c_{4}(\alpha)$ are quantities depending only on $\alpha$ from Theorem 1.5 . Using the Berkes-Philipp lemma (1979, Lemma A1, p. 53) and (9.2), we can suppose without loss of generality that

$$
\begin{aligned}
\tilde{X}_{m} & =2^{-M / 2}\left(X_{(m-1) \cdot 2^{M}+1}+\cdots+X_{m \cdot 2^{M}}\right), \\
\tilde{Y}_{m} & =2^{-M / 2}\left(Y_{(m-1) \cdot 2^{M}+1}+\cdots+Y_{m \cdot 2^{M}}\right),
\end{aligned}
$$$$
m=1, \ldots, 2^{N-M}
$$

where $\left\{X_{1}, \ldots, X_{2^{N}}\right\}$ and $\left\{Y_{1}, \ldots, Y_{2^{N}}\right\}$ are two collections of mutually independent random vectors with $\mathcal{L}\left(X_{k}\right)=\mathcal{L}\left(\xi_{k}\right), \mathcal{L}\left(Y_{k}\right)=\mathcal{N}(0, I)$, for $k=1, \ldots, 2^{N}$.

Denote

$$
\begin{aligned}
& S_{j}=X_{1}+\cdots+X_{j}, \quad j=1, \ldots, 2^{N} ; \quad S_{0}=T_{0}=0 \\
& T_{j}=Y_{1}+\cdots+Y_{j},
\end{aligned}
$$

and, by analogy with the proof of Theorem 6.4 ,

$$
\begin{aligned}
& \Delta_{1}=\max _{0 \leq k<2^{N-M}} \max _{1 \leq s \leq 2^{M}}\left|S_{k \cdot 2^{M}+s}-S_{k \cdot 2^{M}}\right|, \\
& \Delta_{2}=\max _{0 \leq k<2^{N-M}} \max _{1 \leq s \leq 2^{M}}\left|T_{k \cdot 2^{M}+s}-T_{k \cdot 2^{M}}\right|, \\
& \Delta_{3}=\max _{1 \leq k \leq 2^{N-M}}\left|S_{k \cdot 2^{M}}-T_{k \cdot 2^{M}}\right|,
\end{aligned}
$$


where the maximums are taken over integer values of $k$ and $s$. It is evident that from (1.1), (9.6)-(9.9) it follows that

$$
\Delta(X, Y) \leq \Delta_{1}+\Delta_{2}+\Delta_{3} .
$$

Moreover, the relations $(9.5),(9.6),(9.9)$, imply

$$
\Delta_{3}=2^{M / 2} \Delta(\tilde{X}, \tilde{Y}) .
$$

It is clear that we can take $c_{3}(\alpha)<1 / 2$. From (9.4) and from the conditions of Theorem 9.1 it follows that $0<b \tau_{1}<1 / 2$. Recall that Gaussian distributions from $\mathcal{G}_{d}$ belong to all classes $\mathcal{A}_{d}(\tau)$. Using Lemma 2.11 with $t=b, \tau=\tau_{1}$, $B^{2}=2^{M}$, we have

$$
\mathbb{E} e^{b \Delta_{l}} \leq 2^{N-M} \cdot 3 d e, \quad l=1,2 .
$$

Now the statement of Theorem 9.1 can be easily derived from (9.1), (9.3), (9.4) and (9.10)-(9.12) by an application of the Hölder inequality to the exponential moment $\mathbb{E} \exp (b \Delta(X, Y) / 3)$.

For the proof of Theorem 1.3 we need the following Lemma 9.2.

Lemma 9.2. Let $F=\mathcal{L}(\xi) \in \mathcal{A}_{d}(1), \mathbb{E} \xi=0$ and $\operatorname{cov} \xi=I$. Then there exists a random vector $\eta$ and absolute positive constants $c_{69}, c_{70} \geq 1$ such that

$$
\begin{gathered}
\mathbb{E} \eta=\mathbb{E} \xi=0, \quad \operatorname{cov} \eta=\operatorname{cov} \xi=I, \\
\mathbb{P}\left\{|\eta| \leq c_{69}(1+\log d)\right\}=1, \quad \mathcal{L}(\eta) \in \mathcal{A}_{d}\left(c_{70}\right),
\end{gathered}
$$

and, for all $u, v, w \in \mathbb{R}^{d}$,

$$
\mathbb{E}\langle\eta, u\rangle\langle\eta, v\rangle\langle\eta, w\rangle=\mathbb{E}\langle\xi, u\rangle\langle\xi, v\rangle\langle\xi, w\rangle .
$$

The following Lemma 9.3 (due to Yurinskii (1978), see Sazonov (1981, Lemma 3, p. 84)) will be used in the proof of Lemma 9.2 .

LEMMA 9.3. Let $0<\gamma<1$ and let the random variable $\mu$ take values $\frac{1}{2 \gamma}\left(1 \pm\left(1+4 \gamma^{3}\right)^{1 / 2}\right)$ with probabilities $\frac{1}{2} \mp \frac{1}{2\left(1+4 \gamma^{3}\right)^{1 / 2}}$. Then $\mathbb{E} \mu=0$, $\mathbb{E} \mu^{2}=\gamma, \mathbb{E} \mu^{3}=1$.

Proof of Lemma 9.2. Denote

$$
R=4 c_{71}(1+\log d),
$$

where an absolute positive constant $c_{71}$ is so large as it will be necessary for the arguments below. Define the "cubic layers" $A_{j}$ by

$$
A_{j}=\left\{x \in \mathbb{R}^{d}: R(j-1) \leq|x|<R j\right\}, \quad j=1,2, \ldots,
$$

and represent the distribution $F$ as the mixture

$$
F=\sum_{j=1}^{\infty} q_{j} G_{j}
$$

where

$$
q_{j}=F\left\{A_{j}\right\}, \quad G_{j} \in \mathcal{F}_{d}, \quad G_{j}\left\{A_{j}\right\}=1, \quad j=1,2, \ldots
$$


Below we shall denote by $\xi_{j}$ the random vectors with distributions

$$
\mathcal{L}\left(\xi_{j}\right)=G_{j}, \quad j=1,2, \ldots
$$

Lemma 2.11 implies that, for $x \geq 1$,

$$
\mathbb{P}\{|\xi| \geq x\} \leq 2 d e^{-x / 4} .
$$

Using $(9.15),(9.16),(9.18),(9.20)$, we obtain

$$
\begin{aligned}
q_{j} \leq \mathbb{P}\{|\xi| \geq R(j-1)\} & \leq 2 d \exp \left(-\frac{R(j-1)}{4}\right)=\frac{2 d}{\left(e^{c_{71}} d^{c_{71}}\right)^{j-1}} \\
& <\frac{1}{d^{3} j^{3} R^{2}(4 d)^{j}}, \quad j=2,3, \ldots, \quad
\end{aligned}
$$

if $c_{71}$ is sufficiently large.

Below we define a transform of $d$-dimensional distributions which will be denoted for $H=\mathcal{L}(\zeta) \in \mathcal{F}_{d}, r>0$ by $H^{(r)}$. Suppose that $\mu$ is a random variable which is independent of $\zeta$ and has the distribution described in Lemma 9.2 with $\gamma=1-\frac{1}{8 d}$. According to Lemma 9.2, we have

$$
\mathbb{E} \mu=0, \quad \mathbb{E} \mu^{2}=1-\frac{1}{8 d}, \quad \mathbb{E} \mu^{3}=1, \quad \mathbb{P}\{|\mu| \leq 2\}=1 .
$$

Define

$$
s=q_{1}+\sum_{j=2}^{\infty} d^{3} j^{3} q_{j}
$$

The quantity $s$ can be estimated with the help of (9.15), (9.17), (9.18), (9.21), (9.23) as follows:

$$
1=\sum_{j=1}^{\infty} q_{j} \leq s \leq 1+\sum_{j=2}^{\infty} d^{3} j^{3} q_{j}<1+\sum_{j=2}^{\infty} \frac{1}{(4 d)^{j}}<1+\frac{1}{8 d} .
$$

Below we shall use the notation $H^{(r)}$ for

$$
H^{(r)}=\mathcal{L}\left(s^{1 / 3} \mu r^{-1} \zeta\right) .
$$

Consider the probability measure

$$
F_{1}=s^{-1}\left(q_{1} G_{1}^{(1)}+\sum_{j=1}^{\infty} d^{3} j^{3} q_{j} G_{j}^{(d j)}\right) .
$$

Let the random vector $\eta_{1}$ have distribution $\mathcal{L}\left(\eta_{1}\right)=F_{1}$. The relations $(9.17)$, (9.19), (9.22), (9.25), (9.26) imply

$$
\mathbb{E} \eta_{1}=\int_{\mathbb{R}^{d}} x F_{1}\{d x\}=s^{-1}\left(q_{1} s^{1 / 3} \mathbb{E} \mu \mathbb{E} \xi_{1}+\sum_{j=1}^{\infty} d^{2} j^{2} q_{j} s^{1 / 3} \mathbb{E} \mu \mathbb{E} \xi_{j}\right)=0
$$


and, for all $u, v, w \in \mathbb{R}^{d}$,

$$
\begin{aligned}
\mathbb{E}\left\langle\eta_{1}, u\right\rangle\left\langle\eta_{1}, v\right\rangle\left\langle\eta_{1}, w\right\rangle & =s^{-1} \sum_{j=1}^{\infty} d^{3} j^{3} q_{j}\left(\frac{s^{1 / 3}}{d j}\right)^{3} \mathbb{E} \mu^{3} \mathbb{E}\left\langle\xi_{j}, u\right\rangle\left\langle\xi_{j}, v\right\rangle\left\langle\xi_{j}, w\right\rangle \\
& =\sum_{j=1}^{\infty} q_{j} \mathbb{E}\left\langle\xi_{j}, u\right\rangle\left\langle\xi_{j}, v\right\rangle\left\langle\xi_{j}, w\right\rangle \\
& =\int_{\mathbb{R}^{d}}\langle x, u\rangle\langle x, v\rangle\langle x, w\rangle F\{d x\}
\end{aligned}
$$

Furthermore, from $(9.15),(9.16),(9.18),(9.22),(9.24)-(9.26)$ it follows that

$$
\mathbb{P}\left\{\left|\eta_{1}\right|>3 R\right\}=F_{1}\{\{x:|x|>3 R\}\}=0 .
$$

Below we shall denote by $t$ an arbitrary vector $t \in \mathbb{R}^{d}$ with $\|t\|=1$. Using (9.17)-(9.19) and taking into account that $\operatorname{cov} F=\operatorname{cov} \xi=I$, we see that

$$
q_{1} \mathbb{E}\left\langle\xi_{1}, t\right\rangle^{2}=\int_{\mathbb{R}^{d}}\langle x, t\rangle^{2} q_{1} G_{1}\{d x\} \leq \int_{\mathbb{R}^{d}}\langle x, t\rangle^{2} F\{d x\}=\mathbb{E}\langle\xi, t\rangle^{2}=1 .
$$

The relations $(9.17),(9.19),(9.22),(9.24)-(9.26),(9.30)$ imply

$$
\begin{aligned}
\mathbb{E}\left\langle\eta_{1}, t\right\rangle^{2} & =s^{-1}\left(q_{1} s^{2 / 3} \mathbb{E} \mu^{2} \mathbb{E}\left\langle\xi_{1}, t\right\rangle^{2}+\sum_{j=2}^{\infty} d^{3} j^{3} q_{j}\left(\frac{s^{1 / 3}}{d j}\right)^{2} \mathbb{E} \mu^{2} \mathbb{E}\left\langle\xi_{j}, t\right\rangle^{2}\right) \\
& =s^{-1 / 3} \mathbb{E} \mu^{2}\left(q_{1} \mathbb{E}\left\langle\xi_{1}, t\right\rangle^{2}+\sum_{j=2}^{\infty} d j q_{j} \mathbb{E}\left\langle\xi_{j}, t\right\rangle^{2}\right) \\
& \geq s^{-1} \mathbb{E} \mu^{2} \sum_{j=1}^{\infty} q_{j} \mathbb{E}\left\langle\xi_{j}, t\right\rangle^{2}=s^{-1} \mathbb{E} \mu^{2} \mathbb{E}\langle\xi, t\rangle^{2} \geq 1-\frac{1}{4 d} .
\end{aligned}
$$

According to $(9.16),(9.18),(9.19),(9.21)$, we have

$$
\sum_{j=2}^{\infty} d j q_{j} \mathbb{E}\left\langle\xi_{j}, t\right\rangle^{2} \leq \sum_{j=2}^{\infty} d j q_{j} \mathbb{E}\left\|\xi_{j}\right\|^{2} \leq d^{2} R^{2} \sum_{j=2}^{\infty} j^{3} q_{j}<\sum_{j=2}^{\infty} \frac{1}{(4 d)^{j}}<\frac{1}{8 d}
$$

From $(9.22),(9.24),(9.27),(9.30)-(9.32)$ it follows that

$$
\mathbb{E}\left\langle\eta_{1}, t\right\rangle^{2} \leq 1-\frac{1}{64 d^{2}}<1
$$

and, hence, the maximal eigenvalue of the covariance operator $D_{1}=\operatorname{cov} \eta_{1}$ does not exceed 1. Let $D_{2}=I-D_{1}$. From $(9.27),(9.31),(9.33)$ one can easily derive that

$$
0<\left\|D_{2}^{1 / 2} t\right\|^{2}=\left\langle D_{2} t, t\right\rangle=\langle I t, t\rangle-\left\langle D_{1} t, t\right\rangle=1-\mathbb{E}\left\langle\eta_{1}, t\right\rangle^{2} \leq \frac{1}{4 d}
$$


Therefore, $D_{2}$ can be considered as covariance operator. Let $\eta_{2}$ be the random vector which is independent of $\eta_{1}$ and

$$
\mathcal{L}\left(\eta_{2}\right)=\mathcal{L}\left(\sqrt{2} D_{2}^{1 / 2} K\right)
$$

where $K$ is the vector from Definition 6.3. From (9.34), (9.35) it follows that

$$
\mathbb{E} \eta_{2}=0, \quad \operatorname{cov} \eta_{2}=D_{2}, \quad \mathbb{P}\left\{\left\|\eta_{2}\right\| \leq 1\right\}=1,
$$

and, for all $u, v, w \in \mathbb{R}^{d}$,

$$
\mathbb{E}\left\langle\eta_{2}, u\right\rangle\left\langle\eta_{2}, v\right\rangle\left\langle\eta_{2}, w\right\rangle=0
$$

(we use that the vector $K$ has symmetric distribution).

Now we define the vector $\eta$ by $\eta=\eta_{1}+\eta_{2}$. The first of the relations (9.13) follows now from $(9.27),(9.36)$. The second one is a simple consequence of $(9.34),(9.36)$. The third can be easily derived from (9.15), $(9.29),(9.36)$. The simplest way to prove the fourth relation in $(9.13)$ is to use (6.14), Lemma 2.5 and the connection between classes $\mathcal{A}_{d}(\tau)$ and classes of distributions satisfying Bernstein-type inequality conditions (see Zaitsev (1986, p. 205; 1987, 1988)). This proof is elementary and therefore omitted. Finally, it is evident that the relation (9.14) follows from $(9.27)$, (9.28), (9.36), (9.37).

Proof of Theorem 1.3. Without loss of generality we assume that $n=2^{N}$, where $N$ is some integer. Let the integer $M$ be defined by the relation

$$
\tau^{2} \leq 2^{M}<2 \tau^{2}
$$

Let $M \leq N$. Denote

$$
\widetilde{\xi}_{m}=2^{-M / 2}\left(\xi_{(m-1) \cdot 2^{M}+1}+\cdots+\xi_{m \cdot 2}\right), \quad m=1, \ldots, 2^{N-M} .
$$

From the conditions of Theorem 1.3 and from the properties of classes $\mathcal{A}_{d}(\tau)$ it follows that $\mathcal{L}\left(\widetilde{\xi}_{m}\right) \in \mathcal{A}_{d}(1), \mathbb{E} \widetilde{\xi}_{m}=0$ and cov $\widetilde{\xi}_{m}=I$. According to Lemma 9.2 , there exist random vectors $\tilde{\eta}_{m}$ satisfying the relation

$$
\mathbb{P}\left\{\left|\widetilde{\eta}_{m}\right| \leq \rho\right\}=1, \quad \rho=c_{69}(1+\log d), \quad \mathcal{L}\left(\widetilde{\eta}_{m}\right) \in \mathcal{A}_{d}\left(c_{70}\right), \quad c_{70} \geq 1,
$$

and having the same moments of the first three orders as $\widetilde{\xi}_{m}$. Thus, we see that the conditions of Theorem 9.1 are fulfilled with $\tau_{1}=\tau, \tau_{2}=1$, $\tau_{3}=c_{70}, \lambda=\rho$. Therefore, for any $\beta>0$ one can construct on a probability space a sequence of independent random vectors $X_{1}, \ldots, X_{n}$ and a corresponding sequence of independent Gaussian random vectors $Y_{1}, \ldots, Y_{n}$ so that $\mathcal{L}\left(X_{k}\right)=\mathcal{L}\left(\xi_{k}\right), k=1, \ldots, n$, and

$$
\mathbb{E} \exp \left(\frac{c_{5}(\beta) \Delta(X, Y)}{c_{70}^{2} d^{3} \tau \rho}\right) \leq \exp \left(c_{6}(\beta)\left(c_{70} d^{3 / 2} \rho\right)^{3 / 2+\beta} L\left(n / \tau^{2}\right)\right) .
$$

The relations (9.40) and (9.41) imply the statement of Theorem 1.3 in the case $M \leq N$. 
Let now $M>N$. Then we take the vectors $\left\{X_{1}, \ldots, X_{n} ; Y_{1}, \ldots, Y_{n}\right\}$ with needed distributions to be mutually independent. From (9.38) it follows that $n=2^{N}<2 \tau^{2}$. Recall that all $d$-dimensional Gaussian distributions belong to classes $\mathcal{A}_{d}(\tau)$ for all $\tau \geq 0$. Using the completeness of $\mathcal{A}_{d}(\tau)$ with respect to convolution and applying Lemma 2.11 with $t=\frac{1}{3 \tau}, B^{2}=2^{N+1}$, we obtain the inequality $\mathbb{E} \exp (\Delta(X, Y) / 3 \tau) \leq 3 d e^{2}$, which implies the statement of Theorem 1.3 in the case $M>N$.

Proof of Theorem 1.6. It repeats the proof of Theorem 1.3. The only difference is in the construction of the vectors $\widetilde{\eta}_{m}$. Let $n=2^{N}$. Assume that the integer $M \leq N$ and the vectors $\widetilde{\xi}_{m}, m=1, \ldots, 2^{N-M}$, are defined by (9.38) and (9.39). From the conditions of Theorem 1.6 it follows that, for all $u, v, w \in \mathbb{R}^{d}$,

$$
\mathbb{E}\left\langle\widetilde{\xi}_{m}, u\right\rangle\left\langle\widetilde{\xi}_{m}, v\right\rangle\left\langle\widetilde{\xi}_{m}, w\right\rangle=0, \quad m=1, \ldots, 2^{N-M} .
$$

Consider the random vectors $\tilde{\eta}_{m}, m=1, \ldots, 2^{N-M}$, with distributions $\mathcal{L}\left(\widetilde{\eta}_{m}\right)=\mathcal{L}(\sqrt{2} K)$, where $K$ is the vector with symmetric distribution involved in Definition 6.3 . It is clear that the random vectors $\widetilde{\eta}_{m}$ have the same moments of the first three orders as $\widetilde{\xi}_{m}, \mathbb{P}\left\{\left|\widetilde{\eta}_{m}\right| \leq \sqrt{2}\right\}=1, \mathcal{L}\left(\widetilde{\eta}_{m}\right) \in$ $\mathcal{A}_{d}\left(\sqrt{2} c_{14}\right)$. The last inclusion follows from (6.14) and from the properties of classes $\mathcal{A}_{d}(\tau)$. Thus, we see that the conditions of Theorem 9.1 are fulfilled with $\tau_{1}=\tau, \tau_{2}=1, \tau_{3}=\sqrt{2} c_{14}, \lambda=\sqrt{2}$. An application of Theorem 9.1 completes the proof of Theorem 1.6 in the case $M \leq N$. The case $M>N$ can be considered as in the proof of Theorem 1.3.

\section{ACKNOWLEDGMENT}

The main part of this research was done in Bielefeld University. I would like to thank Professor Friedrich Götze for his hospitality during my stay in Bielefeld. I am grateful to V. Bentkus, S. G. Bobkov, U. Einmahl, F. Götze, A. I. Sakhanenko and B. A. Zalesskii (who attracted the author's attention to the book Sazonov (1981)) for useful discussions. I am especially grateful to the referee who carefully read the text and proposed a number of refinements of proofs and improvements of presentation (in particular, the use of Doob's inequality).

\section{BIBLIOGR APHIE}

BÁRtFai, P. (1966), Die Bestimmung der zu einem wiederkehrenden Prozess gehörenden Verteilungfunktion aus den mit Fehlern behafteten Daten einer einzigen Realisation, Studia Sci. Math. Hungar. 1 161-168.

BERgER, E. (1982), Fast sichere Approximation von Partialsummen unabhängiger und stationärer ergodischer Folgen von Zufallsvectoren, Dissertation, Universität Göttingen.

Berkes, I., Philipp, W. (1979), Approximation theorems for independent and weakly dependent random vectors, Ann. Probab. 7 29-54.

Borovkov, A. A. (1973), On the rate of convergence in the invariance principle, Theor. Probab. Appl. $18207-225$.

Csörgő, M., RÉvÉsz, P. (1975), A new method to prove Strassen type laws of invariance principle. I; II, Z. Wahrscheinlichkeitstheor. verw. Geb. 31 255-259; 261-269. 
Csörgö, M., RÉvÉsz, P. (1981), Strong approximations in probability and statistics, Academic Press.

Csörgő, S., HALL, P. (1984), The Komlós-Major-Tusnády approximations and their applications, Austral. J. Statist. 26 189-218.

Doob, J. L. (1953), Stochastic processes, Wiley.

EINMAHL, U. (1986), A refinement of the KMT-inequality for partial sum strong approximation, Techn. Rep. Ser. Lab. Res. Statist. No. 88. Carleton University, University of Ottawa.

Einmaht, U. (1987a), A useful estimate in the multidimensional invariance principle, Probab. Theor. Rel. Fields 76 81-101.

EinMahL, U. (1987b), Strong invariance principles for partial sums of independent random vectors, Ann. Probab. 15 1419-1440.

Einmahl, U. (1989), Extensions of results of Komlós, Major and Tusnády to the multivariate case, J. Multivar. Anal. 28 20-68.

Götze, F., ZAitsev, A. Yu. (1997), Multidimensional Hungarian construction for almost Gaussian smooth distributions, Preprint 97 - 071 SFB 343, Universität Bielefeld.

Komlós, J., Ma.Jor, P., Tusnády, G. (1975; 1976), An approximation of partial sums of independent RV'-s and the sample DF. I; II, Z. Wahrscheinlichkeitstheor. verw. Geb. 32 111-131; 34 34-58.

Massart, P. (1989), Strong approximation for multivariate empirical and related processes, via KMT construction, Ann. Probab. 17 266-291.

Philipp, W. (1979), Almost sure invariance principles for sums of $B$-valued random variables, Lect. Notes in Math. 709 171-193.

Prokhorov, Yu. V. (1956), Convergence of random processes and limit theorem of probability theory, Theor. Probab. Appl. 1 157-214.

Rosenblatt, M. (1952), Remarks on a multivariate transformation, Ann. Math. Statist. 23 470-472.

SAKHANENKO, A. I. (1984), Rate of convergence in the invariance principles for variables with exponential moments that are not identically distributed, In: Trudy Inst. Mat. SO AN SSSR, Nauka, Novosibirsk, 3 4-49 (in Russian).

SAzONOV, V. V. (1981), Normal approximation - some recent advances, Lect. Notes in Math. 879 .

SHAO, QI-MAN (1995), Strong approximation theorems for independent random variables and their applications, J. Multivar. Anal. 52 107-130.

SkorokHoD, A. V. (1961), Studies in the theory of random processes, Univ. Kiev Press (in Russian, Engl. transl. (1965), Addison-Wesley).

STRASSEN, V. (1964), An invariance principle for the law of iterated logarithm, $Z$. Wahrscheinlichkeitstheor, verw. Geb. $3211-226$.

YuRINSkiI, V. V. (1978), On the error of the Gaussian approximation to the probability of a ball, Unpublished manuscript.

Zaitsev, A. Yu. (1986), Estimates of the Lévy-Prokhorov distance in the multivariate central limit theorem for random variables with finite exponential moments, Theor. Probab. Appl. $31203-220$.

ZAITSEV, A. Yu. (1987), On the Gaussian approximation of convolutions under multidimensional analogues of S. N. Bernstein inequality conditions, Probab. Theor. Rel. Fields 74 535-566.

ZAitsev, A. Yu. (1988), On the connection between two classes of probability distributions, In: Rings and modulus. Limit theorems of probability theory. Vol. 2, Leningrad University Press, 153-158 (in Russian).

ZAITSEV, A. YU. (1995a), Multidimensional version of the Hungarian construction, In: Vtoraya Vserossiiskaya shkola-kollokvium po stochasticheskim metodam. Ioshkar-Ola, 1995. Tezisy dokladov, TVP, Moskva, 54-55 (in Russian).

Zaitsev, A. YU. (1995b), Multidimensional version of the results of Komlós, Major and Tusnády for vectors with finite exponential moments, Preprint $95-055$ SFB 343, Universität Bielefeld.

Zaitsev, A. Yu. (1996a), An improvement of U. Einmahl estimate in the multidimensional invariance principle, In: Probability Theory and Mathematical Statistics. 
Proceedings of the Euler Institute Seminars Deducated to the Memory of Kolmogorov. I. A. Ibragimov and A. Yu. Zaitsev eds. Gordon and Breach, 109-116.

Zaitsev, A. Yu. (1996b), Estimates for quantiles of smooth conditional distributions and multidimensional invariance principle, Siberian Math. J. 37 807-831 (in Russian).

Zaitsev, A. Yu. (1997), Multidimensional variant of the Komlós, Major and Tusnády results for vectors with finite exponential moments, Dokl. Math. 56 935-937.

St. Petersburg Branch of Steklov Mathematical Institute, Fontanka 27, St. Petersburg 191011, Russia zaitsev@pdmi.ras.ru 